\title{
Lipid Droplets Degradation by Autophagy Connects Mitochondria Metabolism to PROX1-driven Expression of Lymphatic Genes and Lymphangiogenesis
}

\section{Odeta Mece}

University of Leuven (KU Leuven)

\section{Diede Houbaert}

University of Leuven (KU Leuven)

Tania Durré

Liege University

Hannelore Maes

University of Leuven (KU Leuven)

Marco Schaaf

University of Leuven (KU Leuven)

\section{Sanket More}

University of Leuven (KU Leuven)

\section{Maarten Ganne}

University of Leuven (KU Leuven)

Melissa García-Caballero

University of Leuven (KU Leuven)

\section{Mila Borri}

University of Leuven (KU Leuven)

Jelle Verhoeven

University of Leuven (KU Leuven)

\section{Silvia Blacher}

\section{Liege University}

\section{Bart Ghesquière}

University of Leuven (KU Leuven)

\section{Mieke Dewerchin}

Laboratory of Angiogenesis and Vascular Metabolism https://orcid.org/0000-0002-0382-9346 Johannes Swinnen

Department of Oncology, Laboratory of Lipid Metabolism and Cancer, LKI - Leuven Cancer Institute, KU Leuven - University of Leuven, Leuven https://orcid.org/0000-0002-7720-5077

\section{Stefan Vinckier}


University of Leuven (KU Leuven)

\section{Maria Soengas}

Spanish National Cancer Research Centre https://orcid.org/0000-0003-0612-6299

\section{Peter Carmeliet}

University of Leuven (KU Leuven)

\section{Agnès Noel}

Liege University

Patrizia Agostinis ( $\square$ patrizia.agostinis@kuleuven.be)

University of Leuven (KU Leuven)

\section{Article}

Keywords: autophagy, lipid metabolism, mitochondria, lipophagy, lymphatic endothelial cells, lymphangiogenesis, acetate

Posted Date: April 28th, 2021

DOI: https://doi.org/10.21203/rs.3.rs-403701/v1

License: (c) (7) This work is licensed under a Creative Commons Attribution 4.0 International License. Read Full License 
LIPID DROPLETS DEGRADATION BY AUTOPHAGY CONNECTS MITOCHONDRIA METABOLISM TO

\section{PROX1-DRIVEN EXPRESSION OF LYMPHATIC GENES AND LYMPHANGIOGENESIS}

4 Odeta Meçe $e^{1,2}$, Diede Houbaert ${ }^{1,2}$, Tania Durré ${ }^{3}$, Hannelore Maes ${ }^{1}$, Marco Schaaf ${ }^{1,2}$, Sanket More ${ }^{1,2}$, 5 Maarten Ganne ${ }^{1,2}$, Melissa García-Caballero ${ }^{4,5}$, Mila Borri ${ }^{4,5}$, Jelle Verhoeven ${ }^{1,2}$, Silvia Blacher ${ }^{3}$, Bart

6 Ghesquière ${ }^{6,7}$, Mieke Dewerchin ${ }^{4,5}$, Johan V. Swinnen ${ }^{8}$, Stefan Vinckier ${ }^{4,5}$, María S. Soengas ${ }^{9}$, Peter

7 Carmeliet ${ }^{4,5}$, Agnès Noel $^{3}$, Patrizia Agostinis ${ }^{1,2 *}$

$8{ }^{1}$ Cell Death Research and Therapy Group, Department of Cellular and Molecular Medicine, KU Leuven,

9 Herestraat 49, 3000 Leuven, Belgium

$10 \quad{ }^{2}$ VIB Center for Cancer Biology Research, 3000 Leuven, Belgium

$11{ }^{3}$ Laboratory of Tumor and Development Biology, GIGA (GIGA-Cancer), Liege University, B23, Avenue

12 Hippocrate 13, 4000, Liege, Belgium

$13{ }^{4}$ Laboratory of Angiogenesis and Vascular Metabolism, VIB Center for Cancer Biology, VIB, Leuven,

14 Belgium

$15{ }^{5}$ Laboratory of Angiogenesis and Vascular Metabolism, Department of Oncology, Leuven Cancer 16 Institute, KU Leuven, Leuven, Belgium

$17{ }^{6}$ Metabolomics Expertise Center, VIB Center for Cancer Biology, VIB, Leuven, Belgium.

$18{ }^{7}$ Metabolomics Expertise Center, Department of Oncology, KU Leuven, Leuven, Belgium

$19{ }^{8}$ Laboratory of Lipid Metabolism and Cancer, Department of Oncology, KU Leuven, Leuven, Belgium

$20{ }^{9}$ Melanoma Laboratory, Molecular Oncology Programme, Spanish National Cancer Research Centre

21 (CNIO), Madrid, 28029, Spain

23 *correspondence to: Patrizia Agostinis; patrizia.agostinis@kuleuven.be

Keywords: autophagy, lipid metabolism, mitochondria, lipophagy, lymphatic endothelial cells, 
29 Autophagy has an emerging vasculoprotective role but whether and how it regulates lymphatic

30 endothelial cells (LEC) and lymphangiogenesis is unknown. Here, we show that genetic deficiency of

31 autophagy in LEC impairs the responses to VEGF-C and injury-driven corneal lymphangiogenesis. Loss

32 of autophagy compromises expression of lymphatic markers, affects mitochondrial dynamics and

33 causes an accumulation of lipid droplets (LDs) in LEC and lymphatic vessels in vivo. When LDs

34 accumulate because lipophagy is impaired, mitochondrial ATP production, fatty acid oxidation (FAO),

35 acetyl-COA/CoA ratio and expression of lymphangiogenic PROX1 target genes are dwindled. Enforcing

36 mitochondria fusion by silencing dynamin-related-protein 1 (DRP1) in autophagy-deficient LEC fails to

37 affect LDs turnover and lymphatic gene expression, whereas supplementing the acetyl-CoA precursor

38 acetate rescues LEC identity and lymphangiogenesis in LEC-Atg5 $\%$ mice. Our findings reveal that

39 lipophagy in LEC by supporting FAO, preserves a mitochondrial-PROX1 gene expression circuit that

40 ensures LEC identity, responsiveness to lymphangiogenic mediators and lymphangiogenesis. 
Lymphatic vessels are specialized components of the circulation system involved in tissue fluid homeostasis, dietary fat absorption, inflammatory and immune responses [1]. Understanding the mechanisms regulating lymphatic vessel formation, a process known as lymphangiogenesis, is a pressing need since its excessive activation or dysfunction contribute to a variety of disease conditions including inflammation and cancer metastasis, or lymphedema respectively $[1,2]$.

During development, lymphatic endothelial cells (LEC) differentiate from embryonic venous endothelial cells, through a signaling pathway driven by the programmed induction of the transcription factor Prospero Homeobox 1 (PROX1), which increases the expression of the VEGFR3 receptor [3-5]. VEGFR3 receptors are sensors of the pro-lymphangiogenic ligands VEGF-C or VEGF-D in the microenvironment, determining the activation and directional migration of LEC [6]. Several studies have disclosed the essential role of the master transcription factor PROX1 for the maintenance of LEC identity [5, 7]. Beyond initial LEC specification, steady-state expression levels of PROX1 are required to maintain LEC fate determination through the organism's life. However, a full understanding of the molecular mechanisms and pathways tuning the PROX1-driven gene expression program that continues to sustain LEC identity and functional lymphangiogenesis in adulthood, is still lacking [8]. Mounting evidence suggests a fundamental role for autophagy, the main lysosomal pathway for intracellular disposal of aberrant or obsolete cellular components and their recycling, in vascular biology $[10,11]$. Recycling of metabolites through autophagy can either be used for biosynthetic routes, or to support mitochondria metabolism and energy production. Apart from being a general degradative pathway, autophagy can be highly selective [12]. Specific autophagy pathways, guarantee organellar and cellular homeostasis and contribute to redirect metabolite flow, in a cell-type and stress-dependent manner [13]. The vasculoprotective role of autophagy in endothelial cells has been derived mainly from genetic and pharmacological studies in blood vessels [10]. In contrast, whether and how autophagy regulates fundamental aspects of LEC biology and postnatal lymphangiogenesis is unknown. In this study, we demonstrate that autophagy in LEC is required to maintain lymphatic markers, homeostatic lipid droplets (LDs) turnover, mitochondria morphology and metabolic 
balance. Autophagy-mediated LD breakdown (lipophagy) supplies fatty acids to fuel mitochondriamediated FAO and TCA catabolism, which sustains a positive feedback circuit responsible to maintain PROX1 driven genetic program. Genetic loss of autophagy in LEC curtails lymphangiogenesis after corneal injury, a process that is rescued by supplementing the FA precursor acetate in vivo. Together, our study uncovers the crucial role of autophagy in LD homeostasis, mitochondria metabolism and LEC responses to lymphangiogenic cues, and suggests that harnessing autophagy may offer novel therapeutic opportunities to control defective lymphangiogenesis.

\section{Autophagy is required for LEC homeostasis and injury-driven lymphangiogenesis}

To investigate the overall impact of autophagy in lymphatic EC, we first assessed key autophagy parameters in human dermal LEC (from now on referred to as simply LEC) expressing GFP-LC3, upon autophagy blockade. As expected [14], silencing the essential autophagy gene ATG5 (si ATG5; resulting in around $80 \%$ reduction of ATG5 protein levels) (Fig. 1a), reduced baseline GFP-LC3 punctae and LC3B formation (Fig. 1b,c). Addition of chloroquine (CQ), a lysosomotropic drug that is used to assess autophagic flux status by means of preventing autophagosome-lysosome fusion, showed a significantly increased accumulation of GFP-LC3 punctae (Fig. 1b,c), LC3B and p62 (Fig. 1a), indicating that LEC display a constitutive autophagic flux, under replete conditions.

We next evaluated the functional consequences of autophagy blockade in LEC. Compared to their siscrambled controls (si CTRL), ATG5-silenced LEC (si ATG5) displayed a significantly impaired ability to sprout (Fig. 1d-e), proliferate (Fig. 1f) and migrate (Fig. 1g) in response to VEGF-C, without appreciable differences in cell death (Fig. 1h). We next generated hybrid/chimeric spheroids comprised of equal numbers of autophagy-proficient LEC labelled with a green lipophilic tracer dye and ATG5-deprived

93 LEC, labelled with a red tracer dye. This analysis showed that while autophagy-proficient green cells 94 competed to migrate and started to sprout, ATG5-compromised red cells remained encapsulated in 
the spheroid's core (Fig. 1i), further underscoring their inability to proliferate, migrate and respond to sprouting cues. LEC sprouting, proliferation and migration were also repressed by CQ (Extended Data Fig. 1a-d). This indicates that efficient sprouting requires functional autophagy in LEC.

To assess the validity of these findings in vivo, we generated LEC-specific conditional Atg5 knockout mice (LEC-Atg $5^{-1-}$ ) by crossing Agt5 $5^{\mathrm{fl} / \mathrm{fl}}$ mice with Prox1-cre ${ }^{E R T 2}$ mice expressing a tamoxifen-inducible Cre recombinase in LEC. Tamoxifen induction of Cre recombinase resulted in the absence of ATG5 tissue immunoreactivity (Extended Data Fig. 1e), Atg5 gene excision in $\mathrm{Cre}^{+}$LEC-Atg5 ${ }^{--}$mice but not in Cre- Wild Type (WT) mice (Extended Data Fig. 1f) and absence of ATG5 protein expression in LEC isolated from LEC-Atg $5^{-/}$transgenic postnatal mice (Extended Data Fig. 1g). We then used a corneal wound healing model of lymphangiogenesis [15] to assess the relevance of autophagy in the response of lymphatic vessels to physiological cues. In this model of injury-induced lymphangiogenesis, inflammation drives a massive upregulation of proangiogenic factors, which overwhelms the antiangiogenic mechanisms of the cornea and results in a secondary ingrowth of both blood and lymphatic vessels from the limbal area into the corneal center [15].

Adult 8-12 weeks WT and LEC-Atg $5^{--}$mice were subjected to corneal cauterization and lymphangiogenesis was evaluated in the injured corneas 8 days later. Staining of whole mount corneas with the lymphatic marker LYVE1 revealed that LEC-Atg5 ${ }^{-1}$ mice displayed a significantly reduced injury-induced lymphangiogenesis (Fig. 1j). Computerized quantification showed that loss of autophagy in vivo impaired all tested parameters (Fig. 1k), indicating that both the lymphangiogenic response (length, density) and the complexity of the vasculature (branching, end points) were affected. Double staining of blood $\left(\mathrm{CD} 31^{+}\right)$and lymphatic $\left(\mathrm{LYVE1}^{+}\right)$vessels, showed that blood vessel growth was not affected by loss of Atg5 in LEC (Fig. 11-m). Similar lymphatic defects, albeit mitigated, were observed with CQ, which toned-down angiogenesis as previously reported [16] (Extended Data Fig. 1hk).

Collectively, these results reveal that autophagy is essential for LEC homeostasis, response to VEGF-C and injury-induced lymphangiogenesis in vivo. 


\section{Autophagy deficiency impairs expression of lymphatic markers}

123 We next ascertained whether the observed unresponsiveness of autophagy compromised LEC to

124 VEGF-C, could be a consequence of defects in VEGFR3 receptor availability [17]. VEGFR3 protein

125 levels were similarly reduced by the downregulation of ATG5 or by inhibition of lysosomal degradation

126 by CQ (Fig. 2a-b). Likewise, ATG5 knockdown or CQ (Fig. 2a-f) attenuated protein levels of other key

127 lymphatic markers, including LYVE1, the transcription factors (TFs) Nuclear Receptor 2F2 (NR2F2) and

128 PROX1, which control VEGFR3 signaling and LEC identity $[1,9,18,19]$ (Fig. 2d-f). To assess whether 129 these effects were specific to ATG5, we then silenced other essential Atg genes, like Unc-51-like kinase

1301 (ULK1) (Fig. 2g) a protein involved in incipient stages of autophagosome biogenesis [20] or the E1-

131 like activating enzyme ATG7 (Extended Data Fig. 2a) involved in the conjugation of LC3 to PE [20].

132 Genetic interference with these autophagy regulators phenocopied the effects of ATG5 silencing in

133 reducing the protein expression of all tested lymphatic markers (Fig. 2g-j and Extended Data Fig.2a-d).

134 Because compromising the autophagy-lysosomal machinery exerted a global downregulation of key

135 LEC markers, we then evaluated the effects of autophagy depletion on their RNA expression. Matching

136 the effects observed on the protein levels, RNA expression analysis revealed that the knockdown of

137 ATG5 (Fig. 2k-n), ULK1 (Fig. 2o-r), ATG7 (Extended Data Fig. 2e-h) or CQ treatment (Fig. 2k-n) in LEC

138 resulted in a significantly reduced expression of all tested lymphatic markers.

139 Thus, genetic inhibition of the autophagy machinery blunts both the mRNA and protein expression of

140 the key molecular players of LEC identity.

141

\section{Autophagy regulates lipid droplet homeostasis in lymphatic endothelial cells}

143 We next wished to clarify which autophagy-regulated mechanism could explain the observed

144 effects of autophagy inhibition on LEC homeostasis and identity. We first considered whether

145 compromising autophagy altered mitochondria degradation, a pathway known to contribute to

146 differentiation processes [21]. Silencing ATG5 in LEC did not significantly alter outer (TOMM20) or 
147 inner (OPA1) mitochondrial protein levels, mitochondrial transmembrane potential or elevated

148 mitochondrial superoxide levels (Extended Data Fig. 3a-c). This suggests that the homeostatic

149 unbalance caused by the loss of autophagy in LEC may not be a direct consequence of obvious

150 defects in mitochondrial degradation pathways.

151 Because lymphatics are specialized in lipid trafficking and absorption [22], they express higher levels

152 of fatty acid transporters as compared to blood endothelial cells (BEC) [9]. Since we operate under

153 lipid replete conditions, we then posited that loss of constitutive autophagy could affect lipid

154 trafficking or their storage/turnover.

155 Loss of autophagy did not alter the expression of surface FAs transporters or cytosolic FA binding 156 proteins such as CD36 (Extended Data Fig. 3d,e) and FABP4 (Extended Data Fig. 3f,g) respectively. FAs

157 are stored as triglycerides (TGs) in lipid droplets (LDs), fat storage organelles composed of a neutral

158 lipid core surrounded by a phospholipid monolayer embedding a variety of proteins, which are

159 dynamically remodeled to support energy demands and fatty acid oxidation (FAO) [23].

160 To determine whether autophagy regulates LD homeostasis in LEC, we performed lipid staining with

161 BODIPY 493/503, a fluorescent neutral lipid marker. Inhibition of autophagy either by downregulating

162 ATG5 or ULK1 (thus inhibiting the formation of autophagosomes) or treating LEC with CQ (thus blocking

163 Iysosomal degradation of the LD content) led to a significant elevation in LD numbers (Fig. 3a,b and

164 Extended Data Fig. 3h,i). Electron microscopy analysis confirmed the increased accumulation of

165 electron-dense LDs in LEC upon depletion of ATG5 (Extended Data Fig. 3j). CQ treatment increased the

166 presence of LDs encapsulated into autophagosome-like vesicles (Extended Data Fig. 3j), in line with the

167 ability of CQ-to block their fusion with the lysosome. To further confirm that components of the

168 autophagic machinery associated with LDs, we transiently expressed mCherry-LC3 in control cells

169 stained with BODIPY 493/503. Co-localization analysis revealed that a remarkable fraction of LDs was

170 decorated with LC3 (Fig. 3c,d). Moreover, BODIPY 493/503/mCherry-LC3 co-localization was

171 significantly boosted in LEC treated with CQ (Fig. 3c,d). Consistent with their increased LD number,

172 quantitative lipidomics analysis revealed significantly higher levels of TGs in ATG5-silenced LEC 
173 compared to their si CTRL (Fig. 3e), enriched in several species known to accumulate in LDs [24]. CQ-

174 treated LEC displayed a significant increase in the cellular content cholesterol esters (CE) (Extended

175 Data Fig. 3k), which is likely caused by inhibition of lysosomal acid lipase [25], degrading TGs and CE to

176 FAs and cholesterol, by the CQ-mediated alkalinization of the lysosomes[26].

177 Because in our settings LEC are exposed to nutrient/lipid-containing media, LD accumulation upon 178 autophagy inhibition could be a reflection of increased TG synthesis and LD formation. To prevent new 179 LD biogenesis and evaluate only pathways involved in the degradation of pre-existing LDs, we inhibited 180 diglycerides acyltransferases (DGAT) 1 and 2 [27]. Both DGAT1 and DGAT2 esterify diacylglycerol (DAG) 181 to yield TGs, a required step in the biogenesis of LDs [27], but have reported differential functions and 182 localization $[28,29]$. Inhibition of DGAT1 and/or DGAT1/2 decreased to a similar degree (about 50\%) 183 the number of LDs in both si CTRL and in autophagy compromised LEC (Fig. 3f,g). However, the number 184 of LDs in autophagy-depleted LEC still remained higher as compared to their autophagy-replete 185 counterparts under conditions of inhibition of de novo LD formation, uncovering that degradation of 186 pre-existing LDs is dependent on lipophagy. Thus, LD degradation through autophagy or lipophagy [30]

187 is actively engaged in LEC exposed to lipid-containing media and contributes to maintain homeostatic 188 LD turnover.

189 To confirm the importance of autophagy for LD homeostasis, we then assessed whether genetic inhibition of ATG5 affects LDs turnover in vivo under conditions driving lymphangiogenesis. To this end we co-stained lymphatic vessels with LYVE1 and BODIPY 493/503 after corneal injury in WT and LEC-

$192 \operatorname{Atg}^{-1-}$ mice (Fig. 3h). Remarkably, this analysis showed that while ECs lining lymphatic vessels from 193 WT mice contained few LDs, LYVE1 ${ }^{+}$vessels of the LEC-Atg $5^{-/}$mice displayed a significantly increased 194 number of LDs (Fig. 3i).

195 Collectively these data indicate that constitutive autophagy in LEC regulates LD degradation in steady 196 state conditions and in response to injury-induced lymphangiogenesis in vivo. 
200 Ester hydrolysis of TGs liberates free FAs, which can be transported as fatty acyl-CoA into the 201 mitochondria through the outer mitochondrial membrane carnitine-palmitoyl CoA transferase 1 202 (CPT1A). CPT1A converts long-chain fatty acyl-CoA to the corresponding fatty acyl-carnitines for transport into the mitochondria matrix, and thus represents a key step in FAO. Since genetic loss of the CPT1A isoform compromises in vivo lymphangiogenesis [9], we reasoned that autophagy-mediated LD degradation might supply FAs to fuel mitochondria FAO and oxidative phosphorylation in LEC. To investigate this assumption, we measured oxygen consumption rates (OCR) in freshly isolated LEC from wild type or LEC-Atg5 ${ }^{-1}$ mice. A notable decrease in both the basal and maximal OCR, which is linked to the ability of EC to proliferate [31], was observed in LEC isolated from LEC-Atg5 $\%$ mice (Fig. 4a-d). A similar phenotype, although attenuated compared to the chronic inhibition of autophagy by gene deletion, was observed in ATG5-depleted LEC (Extended Data Fig. 4a-d) and LEC treated with CQ 211 (Extended Data Fig. 4e-h). Downregulation of ATG5 did not affect extracellular acidification rate (ECAR) 212 under basal conditions and after 2-deoxy-D-glucose (2-DG) treatment (Figure S4I), as also confirmed 213 by MS analysis showing unchanged total abundancies of lactic acid levels (Extended Data Fig. 4j).

214 We then measured FAO-linked oxygen consumption by evaluating OCR under etomoxir-treated conditions to inhibit CPT1A. Maximal respiration and spare respiratory capacity decreased significantly in autophagy-proficient LEC by the short-term addition of the specific CPT1 blocker etomoxir, to levels

217 similar to those observed in autophagy-compromised cells (Fig. 4e-g). This suggests that a substantial 218 fraction of OCR in steady state conditions is due to oxidation of FAs. Genetic blockade of autophagy in 219 combination to pharmacological inhibition of CPT1A caused additional effects on mitochondrial 220 bioenergetics (Fig. 4e-g). Since etomoxir can have off-target effects [32], we then validated the impact 221 of ATG5 inhibition on mitochondrial FAO, by assessing the rate of $\left[9,10-{ }^{3} \mathrm{H}\right]$-palmitate oxidation. 222 Silencing autophagy caused a significantly reduction (50\% decrease) in the ability of LEC to perform 223 FAO (Fig. 4h). Consistent with the role of FAO in the maintenance of redox homeostasis in EC [33], 224 impairing autophagy increased the levels of oxidized glutathione GSSG (Extended Data Fig. 4k) as 
measured by MS analysis. Furthermore, knockdown of ATG5 also reduced levels of eNOS (Extended

226 Data Fig. 4 I-m), which maintains redox homeostasis in EC by regulating levels of reducing equivalents 227 GSH and NADPH [34]. Thus, autophagy in LEC maintains FAO and cellular redox homeostasis.

228 Next, since mitochondria dynamics and shape are vital parameters of the mitochondrial metabolic and bioenergetic status [35] and an elongated mitochondria network regulates mitochondrial FA uptake

230 [36], we analyzed the mitochondrial network in autophagy proficient and compromised LEC.

231 Immunofluorescence staining of the mitochondrial outer protein TOMM20 revealed that autophagy-

232 proficient LEC displayed an interconnected mitochondrial network (Fig. 4i). In contrast, but in line with

233 their respiratory-defective mitochondria phenotype (Fig. 4a-d), ATG5-depleted LEC displayed a higher 234 index of mitochondrial fragmentation (Fig. 4j), which correlated with the activating Ser616 235 phosphorylation of the main mitochondrial fission regulator DRP1 (Fig. 4k). Pharmacological inhibition 236 of CPT1A by etomoxir resulted also in a highly fragmented mitochondria network (Extended Data Fig. $2374 \mathrm{n}-0)$, again indicating the close inter-relationship between FAO proficiency and mitochondrial shape. 238 Collectively, these results indicate that autophagy is required to sustain mitochondrial FAO and 239 oxidative phosphorylation in LEC.

\section{Lipophagy regulates CPT1A expression and acetyl-CoA levels in LEC}

242 CPT1A has been found to be a target of a PROX1-driven feedback loop in LEC [9], but the homeostatic 243 mechanism that supports this mitochondria-gene expression circuit is unknown. Given that lipophagy 244 supplies FAs to boost FAO and TCA in mitochondria, we then reasoned that loss of LEC-autophagy could 245 result in a concomitant downregulation of CPT1A levels, further impairing the overall capacity of the 246 LEC to utilize FAs as energy source.

247 Silencing of ATG5 significantly reduced protein (Fig. 5a,b) and transcript levels of CPT1A (Fig. 5c) in 248 LEC. A similar trend was observed for the inner mitochondria membrane-associated CPT2A (Extended 249 Data Fig. 5a,c), which participates to the carnitine cycle by converting acylcarnitine back to acyl-CoA 250 for oxidation in the matrix. 
We then tested if the reduced levels of CPT1A and impaired FAO and TCA in autophagy-compromised

252 LEC, affected cytosolic acetyl-CoA, which is generated after export of citrate from the mitochondria to

253 the cytosol and its conversion by ATP citrate lyase (ACLY) in acetyl-CoA. MS analysis revealed that in

254 ATG5-silenced LEC both citrate (Fig. 5d) and acetyl-CoA/CoA (Fig. 5e) levels were significantly reduced.

255 Likewise, etomoxir treatment reduced acetyl-CoA/COA ratios to an extent similar to that observed in

256 autophagy-compromised LEC (Fig. 5e). Thus, reducing the ability of mitochondria to perform efficient

257 FAO consistently dwindled acetyl-CoA/CoA ratio.

258 Because histone $\mathrm{H} 3$ acetylation at lysine 9 ( $\mathrm{H} 3 \mathrm{~K} 9 \mathrm{ac}$ ) by $\mathrm{p} 300$ is a marker of active gene promoters

259 sensitive to acetyl-CoA level [37], which bolsters epigenetic regulation of PROX1 driven transcription

260 of lymphatic genes [9], we then tested if compromising autophagy in LEC reduced H3K9 acetylation. In

261 line, loss of ATG5 led to a significant reduction in H3K9ac levels in LEC (Fig. 5f,g). Furthermore, this

262 effect was phenocopied by silencing ATG7 or ULK1 (Fig. 5h), thus validating the role of bona fide

263 autophagy in sustaining levels of acetyl-CoA for histone $\mathrm{H} 3$ acetylation.

Acetate rescues the mitochondrial-PROX1 transcriptional circuit and functional defects caused by genetic deficiency of autophagy in LEC

267 We next wished to portray the functional link between the mitochondrial metabolic defects and the transcriptional deficit of key lymphatic genes caused by the loss of autophagy.

269 We then investigated if supplementation of acetate, a precursor of acetyl-CoA which does not require

270 a carnitine shuttle mechanism of mitochondrial transport [38], could restore expression of lymphatic 271 markers in autophagy-defective LEC.

272 Acetate supplementation to ATG5-silenced LEC indeed restored the expression of PROX1, VEGFR3 and 273 LYVE1, both at the RNA (Fig. 6a-c) and protein level (Fig. 6d) to that of si CTRL LEC. Similar rescuing 274 effects of acetate on protein and RNA level were observed or upon silencing of ULK1 (Extended Data 275 Fig. 6a-d) or ATG7 (Extended Data Fig. 6e-h). Notably, both the defects in H3K9 acetylation and CPT1A 276 levels observed in LEC deprived of autophagy were reversed by acetate supplementation (Fig. 6d). In 
contrast, providing palmitate, a FA requiring CPT-mediated shuttle to enter mitochondria, failed to 278 rescue LEC markers (Fig. 6e and Extended Data Fig. 6i-I). Thus, PROX-1 lymphatic gene expression in 279 autophagy-incompetent LEC can only be recovered by providing a FA precursor and through a CPT1A280 independent mechanism, further linking LD degradation by autophagy to the metabolic-transcriptional 281 circuit that preserves FAO and the LEC phenotype. Moreover, pharmacological inhibition of ACLY, which is required to convert citrate in acetyl-CoA, curbed both the overall expression of LEC markers in si CTRL and the rescue effect of acetate on ATG5 silenced (Extended Data Fig. $6 \mathrm{~m}$ ). This suggests that acetyl-COA is required to preserve LEC markers and that the action of acetate relies on the epigenetic regulation of PROX-1 transcription.

To further appreciate the functional impact of this metabolic circuit, we then tested whether acetate could restore VEGF-C induced sprouting responses in autophagy-deprived LEC. In si CTRL LEC, acetate had a marginal effect on spheroid sprouting (Fig. $6 f$ and Extended Data Fig. 6n) in combination with VEGF-C. Notably, supplementing acetate restored sprout numbers of autophagy-defective LEC almost to control values (Fig. $6 \mathrm{f}$ and Extended Data Fig. 6n), in line with the recovery of VEGFR3 protein levels 291 (Fig. 6d).

292 In line with an overall improvement in mitochondria function, providing exogenous fuel for FAO reestablished a tubular mitochondrial network characteristic of respiring mitochondria in autophagydefective LEC (Fig. 6g,h). Compared to si CTRL cells and as observed before, LEC with defective autophagy displayed an increased number of LDs, which was not affected by the addition of acetate (Fig. 6i). To clarify whether restoring mitochondrial tubular morphology in ATG5-compromised LECs, was per se sufficient to regain LEC specific markers, we silenced DRP1. Downregulation of DRP1 298 expression (Fig. 6j), marginally affected the mitochondrial network in si CTRL cells while it significantly 299 induced mitochondria fusion in ATG5-depleted LEC (Fig. 6k-I). Restoring the elongated mitochondrial 300 morphology however, did not affect LD's number (Fig. $6 \mathrm{~m}$ ) and did not rescue the mRNA levels of 301 PROX1, VEGFR3 and LYVE1 (Extended Data Fig. 6 o-q). 
302 Thus, when degradation of LDs is inhibited, exogenous provision of acetate recovers the elongated

303 morphology of FAO-proficient mitochondria and fuels the transcriptional lymphatic program. In

304 contrast, the sole rescue of a tubular mitochondrial network in lipophagy-compromised conditions fails

305 to recover LEC identity.

306

307

Acetate rescues injury-driven lymphangiogenesis in LEC-ATG5 knock out mice

308

Given the major rescuing effects of acetate observed in vitro, we then tested if acetate supplementation could restore corneal lymphangiogenesis in the LEC-ATG5 ${ }^{-1}$ mice. While acetate injection in WT mice did not exert significant effects, supplementing acetate to LEC-ATG5 $\%$ mice

311 rescued injury-driven lymphangiogenesis (Fig. 7a). Especially LYVE1 ${ }^{+}$vessel branching, end points and

312 length (Fig. 7b-e) were restored to levels similar to those of WT treated animals. Alongside, acetate 313 supplementation did not have any significant effects on blood vessel formation (Fig. 7f-j). These results 314 show that acetate is able to restore responsiveness of autophagy-deprived LEC to pro315 lymphangiogenic factors promoting migration and proliferation of LEC, under these inflammatory 316 conditions. Acetate did not significantly rescue the milder effects observed after CQ treatment on 317 lymphatic branching and end points (Extended Data Fig. 7a,d-e). This suggests that CQ might inhibit 318 additional (paracrine) inflammatory pathways regulating injury-driven lymphangiogenesis in vivo, 319 which are insensitive to acetate.

320 Collectively these results show that impaired corneal lymphangiogenesis caused by genetic loss of LEC321 autophagy can be reversed in vivo by rescuing metabolic alterations through acetate supplementation 322 (Fig.8).

\section{DISCUSSION}

325 In this study, we provide compelling evidence showing that autophagy in LEC is a constitutive process 326 connecting mitochondria dynamics and metabolism to PROX1-driven genetic program that maintains 327 LEC identity and Iymphangiogenesis in vivo. Impairing the autophagic machinery, results in the 
accumulation of TG- containing LDs and defective mitochondria, which are unable to efficiently

329 perform FAO and to maintain acetyl-CoA levels required to support (epigenetic) regulation of PROX1-

330 target genes. Thus, this study identifies LD degradation by autophagy -or lipophagy- as essential 331 process to sustain postnatal LEC homeostasis and identity.

332 While sequestration of FAs in LDs functions as a buffering mechanism to prevent the potential deleterious effect of free FAs and lipotoxicity $[23,39,40]$, selective lysosomal degradation of LDs during periods of nutrient unavailability, during cell growth or differentiation $[41,42]$ fosters metabolic processes and membrane biosynthesis [23].

Our study shows that genetic deletion of assorted autophagy genes (Atg5, Atg7 and ULK1) in LEC impairs constitutive degradation of LDs, with detrimental effects on LEC functions. Compromising

338 lipophagy results in defective FAO and altered mitochondria dynamics, which interrupt the 339 mitochondrial-PROX1-driven transcriptional circuit responsible for the expression of lymphangiogenic 340 markers. This finding unravels the relevance of this catabolic process for the maintenance of the

341 lymphatic EC phenotype and underscores the intimate but still elusive link between LD degradation, 342 mitochondrial shape and metabolism [35]. While it is well established that the elongated mitochondrial 343 shape is critical for proficient metabolic activity, current insights into the interaction between LDs and 344 mitochondria dynamics are still limited $[23,35]$. Also, the molecular entities operating as lipophagy receptors are still largely undefined. In starved cells, preventing mitochondria fusion resulted in 346 unmetabolised FAs, which were re-routed to LDs in order to avoid lipotoxicity [41].

347 Mechanistically, we show that in autophagy-depleted LEC the supplementation of the FA precursor 348 acetate recovers the elongated mitochondria morphology without altering the accumulation of LDs.

349 On the other hand, rescuing mitochondria elongation by silencing DRP1 in autophagy-compromised 350 LEC does not alter LD accumulation and fails to correct the expression of lymphatic markers. Together 351 these findings support the concept that lipophagy in LEC operates primarily as a mechanism of FA352 supply to the mitochondria and that LD turnover is not secondary to alterations of the mitochondria 353 bioenergetic status. However, further research is required to completely clarify the complex 
interconnection between mitochondria dynamics and LD metabolism $[40,42,43]$ and to completely

355 rule out that LD accumulation in autophagy deficient cells is a consequence of a reduction of CPT1A

356 levels.

357 Given the specialized function of lymphatic vessels in lipid trafficking, which is reflected by the high

358 expression of lipid transporters, it is plausible that LEC become particularly dependent on signaling mechanisms regulating FA storage and degradation to maintain metabolic fitness, homeostasis and LEC specification. It will be of considerable interest to further validate the link between lipophagy and

361 epigenetic regulation through the modulation of the acetyl-CoA availability and to elucidate the 362 underlying molecular mechanisms.

363 The finding that acetate supplementation restores LEC markers and rescues the corneal 364 lymphangiogenesis deficits of the LEC-Atg5 $5^{-1-}$ mice, unravels the key regulatory role that autophagy 365 plays in the metabolic homeostasis of lymphatic vessels. In fact, our study provides mechanistic 366 insights supporting the concept that autophagy in LEC is critical to integrate lipid catabolism, 367 mitochondria FAO and PROX-1-driven transcriptional regulation of lymphatic phenotype.

368 Recent studies show that ketone bodies provided by high-fat, low-carbohydrate ketogenic diet, which 369 are known autophagy stimulators [44, 45], improve lymphangiogenesis after corneal injury and 370 myocardial infarction, and lymphatic vessel function and growth in a mouse model of lymphedema 371 [46]. Hepatic loss of Atg7 or Atg5 significantly impairs LD degradation, FAO and ketone bodies 372 production upon fasting [47], further highlighting the link between tissue-specific catabolism of FAs 373 and autophagy. Considering that LEC-autophagy provides the fuel supply to maintain FAO and 374 ultimately lymphatic proliferation and migration, it is tempting to speculate that dietary or life-style 375 conditions that stimulate autophagy (e.g. caloric restriction, keto-diet, improved exercise) may 376 facilitate restoring lymphatic functions in pathological conditions such as obesity or lymphedema. 
381 Cell Culture and RNA Interference: Human dermal lymphatic endothelial cells (LEC) were commercially

382 purchased from Promocell (C-12217 cultured on dishes precoated with 0.1\% gelatin (Sigma Aldrich) 383 and used between passages 2 and 8. LEC were grown in ECGMV2 added with SupplementMix (C-22211 384 and C-39226, Promocell). SiRNA transient transfection was performed twice on consecutive days using $38540 \mathrm{nM}$, non-targeting siRNA (si CTRL), siRNA against human ATG5 (si ATG5), ULK1 (si ULK1), ATG7 (si ATG7) and DRP1 (si DRP1) purchased from Dharmacon (D-001810, L-004374, L-005049, L-020112 and L-012092 respectively). Treatments with chloroquine (CQ), sodium acetate (AC), etomoxir (Eto), VEGFC, palmitate, diglyceride acyltransferase inhibitor 1 / 2 (DGAT1/2) and ATP citrate lyase inhibitor (ACLYi) (C6628, S2889-250G, E1905, SRP3184, 14464-31-4, PZ0207/ PZ0233 and SML0784 respectively, Sigma Aldrich) for assessing signaling and functional assays were done for $48 \mathrm{~h}$. All cells were maintained in an incubator at $37^{\circ} \mathrm{C}$ with $5 \% \mathrm{CO} 2$ and $95 \%$ air.

393 LC3-GFP transfection: 26000 cells $/ \mathrm{cm}^{2}$ were seeded in a 12 well plate on day one. siRNA 394 transient transfection was performed following the protocol described above. The next day, plasmid 395 (pBABEpuro GFP-LC3, addgene plasmid \#22405) transfection (800 ng/well) was performed with 396 Lipofectamine 2000 (Invitrogen, 16688), following manufacturer's protocol. Cells were fixed with 4\% paraformaldehyde (J19943K2, Thermo Fisher Scientific) 24h after plasmid transfection. Cell nuclei were stained with DAPI (D1306, Thermo Scientific). Fluorescence images were acquired using an inverted microscope (IX83, Olympus). Analysis was performed using NIH ImageJ software.

401 Immunoblot analysis: Cells were lysed in a modified Laemli buffer (125 mM Tris-HCl, pH 6.8 buffer 402 containing $2 \%$ SDS and $20 \%$ glycerol) with the addition of protease and phosphatase inhibitors 403 (A32953, A32957 respectively, Thermo Fisher Scientific). Proteins were separated using SDS-PAGE 404 under reducing conditions, transferred to a nitrocellulose membrane and examined by 405 immunoblotting. Primary antibodies used were rabbit anti-ATG5 (12994S, CST), rabbit anti-ATG7 
406 (8558S , CST), rabbit anti-LC3 (3868S, CST), rabbit anti-GAPDH (2118S, CST), rabbit anti-p62 (p0067, 407 Millipore), rabbit anti- $\beta$-actin (A5441, Sigma-Aldrich), goat anti-LYVE1 (AF2089, R\&D systems), rabbit 408 anti-PROX1 (11067-2, Proteintech), rabbit anti-VEGFR3 (ab154079, Abcam), mouse anti-NR2F2 409 (ab41859, Abcam), rabbit anti-CPT1 (D3B3, CST) antibody, rabbit anti-CPT2 (ab18114, Abcam), rabbit 410 anti-acetyl histone H3 (lysine 9) antibody (9671, CST), rabbit anti-pan-acetyl histone H3 antibody 411 (39139, Active Motif)), mouse anti-CD36 antibody (ab17044, Abcam), mouse anti-ULK1 antibody 412 (ab56344, Abcam), rabbit anti-TOMM20 (BD612278, BD Biosciences), mouse anti-OPA1 (612607, BD 413 Biosciences), mouse anti-ENOS (610297, BD biosciences), rabbit anti-phospho DRP1 (Ser616) (3455S, 414 CST), mouse anti-DRP1 (611113, BD Biosciences) and rabbit anti-FABP4 antibody (2120S, CST). 415 Appropriate secondary antibodies were from Cell Signaling Technology and Thermo Fisher Scientific 416 (Erembodegem, Belgium). Membranes were scanned using the Bio-Rad Chemidoc Imager (Bio-Rad 417 Laboratories N.V.3). Quantification of western blot data was done using ImageLab software.

419 Quantitative-Real-Time PCR: RNeasy Plus mini kit (74136, Qiagen) was used for RNA extraction and 420 reverse transcription kit QuantiTect (205313, Qiagen) for CDNA generation. Gene expression was 421 determined with ORA qPCR Green L mix (QPD0105, HighQu) utilizing the ABI 7,500 machine (Applied 422 Biosystems) and analyzed using the delta delta Ct method. Primer sequences are available in Table 1. Supplementary table: Primer sequences

\begin{tabular}{|l|l|l|}
\hline Gene & Forward primer & Reverse primer \\
\hline Human VEGFR3 & AAGATGTTTGCCCAGCGTAG & GCACTGTGGCATGAGGTCT \\
\hline Human LYVE1 & TGAAGGGGTAGGTGTGATGG & ATGACACCTGGATGGAAAGC \\
\hline Human PROX1 & TCACCTTATTCGGGAAGTGC & GAGCTGGGATAACGGGTATAAA \\
\hline Human NR2F2 & CCATAGTCCTGTTCACCTCAGA & AATCTCGTCGGCTGGTTG \\
\hline Human CPT1A & CAATCGGACTCTGGAACCG & CCGCTGACCACGTTCTTC \\
\hline Human CPT2A & CCACCATGCACTACCAGGA & TGGTGTCTTCAAGTTTGGGAAT \\
\hline
\end{tabular}




\begin{tabular}{|l|l|l|} 
Human ACC1 & GCTGGTCCACATGAACAGG & GCCTTCTGGATATTCAGGACTTT \\
\hline Human HPRT & GACCAGTCAACAGGGGACAT & GTGTCAATTATATCTTCCACAATCAAG \\
\hline Human ATG5 & CAACTTGTTTCACGCTATATCAGG & CACTTTGTCAGTTACCAACGTCA \\
\hline
\end{tabular}

423

424

Spheroid sprouting assay: Spheroids of LEC were generated from 2000 cells in hanging drops in ECGM2 containing $20 \%$ of methylcellulose (M6385, Sigma Aldrich). As previously described in [48], spheroids were embedded in a collagen gel (08-115, Merck, Germany) and cultured in respective medium of LEC. Depending on experimental settings, cells were stimulated with $100 \mathrm{ng} / \mathrm{mL}$ VEGF-C, treated with 25 $\mu \mathrm{M}$ CQ (C6628, Sigma Aldrich) or supplemented with $20 \mathrm{mM} \mathrm{AC}$ and cultured for $48 \mathrm{~h}$ to analyze sprouting. For the mixed spheroids, LEC were collected $48 \mathrm{~h}$ after transfection and labeled with cell membrane labeling lipophilic dyes CellVue ${ }^{\mathrm{TM}}$ Jade and CellVue ${ }^{\mathrm{TM}}$ NIR780 (88-0876-16 and 88-0875-16 respectively, Thermo Fisher Scientific). Spheroids containing equal amounts of si CTRL transfected cells (green) and si ATG5 transfected cells were generated, embedded and analyzed after $48 \mathrm{~h}$. Images were taken in an inverted microscope (IX83, Olympus) and analysis for number of sprouts per spheroid was performed using NIH ImageJ software.

Scratch wound assay: The scratch wound was made on confluent LEC monolayers. Cell plates were washed twice to remove floating cells and treated with $500 \mu \mathrm{g} / \mathrm{ml}$ Mitomycin C (M4287, Sigma Aldrich) to block proliferation. A scratch wound was applied on the confluent LEC monolayer using a $200 \mu \mathrm{L}$ tip. After scratch wounding and immediate photography, the cultures were further incubated for the $24 \mathrm{~h}$ and photographed again. Migration distance was measured with NIH ImageJ software and is expressed as percentage wound closure.

Proliferation assay via Thymidine incorporation: 25000 cells were seeded per well on a 24-well plate. The next day, cells were incubated with $1 \mu \mathrm{Ci} \mathrm{m}^{-1}$ [3H]-thymidine (NET355L005MC, Perkin Elmer) for 
$24 \mathrm{~h}$, fixed with $100 \%$ ethanol at $4{ }^{\circ} \mathrm{C}$ for $15 \mathrm{~min}$ and precipitated using $10 \%$ trichloroacetic acid (T6399,

447 Sigma Aldrich) and lysed with $0.1 \mathrm{~N} \mathrm{NaOH}$. Amounts of [3H]-thymidine incorporated into DNA was 448 measured by scintillation counting (Scintillation counter, Perkin Elmer).

450 Cell death: LEC were trypsinized $48 \mathrm{~h}$ post transfection and incubated with $2 \mu \mathrm{g} / \mathrm{mL}$ propidium iodide 451 (PI) (P4170, Sigma). Dead cells were defined as PI-positive cells using AttuneTM Cytometer (Thermo Fisher Scientific). Quantification was performed using FlowJo software.

BODIPY (493/503) staining: Cells were seeded on coverslips at the point of interest and transfected with mCherry-hLC3B-pcDNA3.1 plasmid, following the protocol described above. After $24 \mathrm{~h}$, plates were washed with PBS (D8537, Sigma Aldrich) and incubated in the dark for 30min with $2 \mu$ M BODIPY 493/503 (D3922, Thermo Fisher Scientific) staining solution prepared in ECGM2. Coverslips were washed 3 times in PBS, fixed in 4\% PFA for $10 \mathrm{~min}$, stained with DAPI for another $5 \mathrm{~min}$, washed in PBS and mounted in a drop of Prolong ${ }^{\circ}$ Gold (P36934, Thermo Fisher Scientific) [49]. Images were acquired on a Nikon C2 Eclipse Ni-E inverted confocal microscope at VIB Bio Imaging Core facility or an inverted microscope (IX83, Olympus). Analysis was performed using NIH ImageJ software. For corneal tissue staining, dissected corneas were washed in PBS followed immediately by staining with $2 \mu \mathrm{M}$ BODIPY 493/503 staining solution prepared in PBS, for 30min in the dark. Corneas were washed for $30 \mathrm{~min}$ at room temperature, fixed in $70 \%$ ethanol for $1 \mathrm{~h}$, washed with PBS, stained with DAPI for $10 \mathrm{~min}$ and flat-mounted on a microscope slide.

Immunostaining TOMM20/BODIPY 493/503: After staining with BODIPY 493/503 as described above, 468 cells were washed twice times in PBS, fixed in 4\% PFA for $15 \mathrm{~min}$, blocked (1X PBS / 5\% normal goat 469 serum (\#5425, CST) / 0.1\% Saponin (S-4521 Sigma Aldrich) for $1 \mathrm{~h}$ and stained overnight at $4{ }^{\circ} \mathrm{C}$ using 470 mouse anti-TOMM20 dilution (1:500) (BD 612278, BD Biosciences) prepared in blocking solution. Cells 471 were washed and incubated with a fluorochrome-conjugated secondary antibody Alexa Fluor 647 
472 (A21235, Thermo Fisher Scientific) for $2 \mathrm{~h}$ in the dark at room temperature. Cells were rinsed with PBS, 473 stained with DAPI for $5 \mathrm{~min}$ and mounted using Prolong ${ }^{\circ}$ Gold Antifade Reagent. Images were acquired 474 on a Nikon C2 Eclipse Ni-E inverted confocal microscope at VIB Bio Imaging Core facility. Mitochondrial 475 morphology was analyzed using the 'Mito-morphology' macro from ImageJ/Fiji Software.

Immunostaining LYVE1/ATG5: Mouse liver tissue samples were immediately fixed in 4\% PFA overnight at $4{ }^{\circ} \mathrm{C}$, dehydrated and embedded in paraffin. Immunostainings were performed using the following primary antibodies: anti-LYVE1 (R\&D systems, AF2125) anti-ATG5 (12994S, CST) and secondary antibodies (Life Technologies). Nuclei were counterstained with DAPI. Fluorescence images were acquired using an inverted microscope (IX83, Olympus).

Mitosox and TMRM analysis via flow cytometry: Prior to staining with TMRM or Mitosox respective positive controls were prepared either by treating with $1 \mu \mathrm{M}$ Antimycin A (A8674, Sigma Aldrich) for $30 \mathrm{~min}$ or $0.5 \mu \mathrm{M}$ FCCP (1528-10, Sanbio) for 20min. Then cells were washed, trypsinized and collected in endothelial growth medium. We stained in parallel for Mitosox $2.5 \mu \mathrm{M}$ and TMRM $20 \mathrm{nM}$ for $1 \mathrm{~h}$. Samples were then washed 2 times with cold PBS and read using AttuneTM Cytometer (Thermo Fisher Scientific). Analysis was performed using FlowJo software.

Seahorse real-time extracellular acidification (ECAR) and oxygen consumption (OCR) test: 30000 491 (human LEC) or 60000 (murine LEC; see below) cells were seeded on Seahorse Xp culture plates 492 (103022-100, Agilent) in Seahorse XF Assay Medium (103335-100, Agilent), pH 7.4 containing 10 mM 493 Glucose (8769,Sigma Aldrich), 1 mM Sodium Pyruvate (S8636,Thermo Fisher Scientific) and 2 mM 494 Glutamine (G7513, Sigma Aldrich), following the manufacturer's instructions. Prior to analysis, cells 495 were maintained for $1 \mathrm{~h}$ in a $\mathrm{CO}_{2}$-free incubator at $37^{\circ} \mathrm{C}$. OCR was measured after serial injections of 8 $496 \mu \mathrm{M}$ Oligomycin (75351, Sigma Aldrich), $4.5 \mu \mathrm{M}$ phenylhydrazone (FCCP, 1528-10, Sanbio) and $5 \mu \mathrm{M}$ 497 Antimycin A (A8674, Sigma Aldrich). ECAR was measured after serial injections of $80 \mathrm{mM}$ glucose, 8 
$\mu \mathrm{M}$ oligomycin and $500 \mathrm{mM}$ 2-deoxy-D-glucose (D-6134, Sigma Aldrich). Analysis was performed using

499 Seahorse Wave Desktop Software (Agilent).

500

501 Mitostress test for B-oxidation via seahorse: $24 \mathrm{~h}$ prior to the assay, cells were put in substrate limited DMEM medium containing $0.5 \mathrm{mM}$ Glucose, $1 \mathrm{mM}$ Glutamine, $0.5 \mathrm{mM}$ L-Carnitine (8400920025, Sigma

503

504

505

506

507

508

509

510 Aldrich) and 1\% fetal bovine albumin (FBS). 45 min prior to the assay, cells were washed and placed in KHB medium supplemented with $2.5 \mathrm{mM}$ glucose, $0.5 \mathrm{mM}$ L-carnitine and $5 \mathrm{mM}$ Hepes (12509079, Gibco), pH 7.4 in a $\mathrm{CO}_{2}$-free incubator at $37^{\circ} \mathrm{C} .40 \mu \mathrm{M}$ Etomoxir (Eto) (E1905, Sigma Aldrich) was added in specific conditions 15 min before the start of the assay. Analysis was performed using Seahorse Wave Desktop Software (Agilent).

Fatty Acid Oxidation assay: After 48h of transfection, LEC were supplemented with $100 \mu \mathrm{M}$ unlabeled palmitate, $50 \mu \mathrm{M}$ carnitine (Sigma-Aldrich) and $2 \mathrm{mCi} / \mathrm{mL}\left[9,10-{ }^{3} \mathrm{H}\right]$-palmitic acid (Perkin Elmer) for $18 \mathrm{~h}$ in complete endothelial growth medium. Then supernatants were collected into glass vials sealed with rubber stoppers. As a readout for the assay ${ }^{3} \mathrm{H}_{2} \mathrm{O}$ was captured in Whatman paper soaked with $\mathrm{H}_{2} \mathrm{O}$ over a period of $48 \mathrm{hr}$ at $37 \mathrm{C}$ [50]. Radioactivity was determined by liquid scintillation counting.

Mouse models: Animal procedures were approved by the Institutional Animal Care and Research Advisory Committee (KU Leuven) (187/2018) and were performed in accordance with the institutional and national guidelines and regulations. Mice from the EC-specific inducible Cre-driver line Prox1$\mathrm{cre}^{\text {ERT2 }}$ [51] were crossed with Atg5 ${ }^{\mathrm{fl} / \mathrm{fl}}$ mice [52] to obtain mice with LEC-specific deletion of the Atg5gene. These lines were on a 100\% C57BL/6 background. For experiments in this study, we used mice expressing Cre (Prox1-Cre+ $\left.{ }^{E R T 2} ; \operatorname{Atg} 5^{f / f f}\right)$, referred to as LEC-Atg5 ${ }^{-1-}$ and their Cre-negative littermates (Prox1-Cre- ${ }^{E R T 2}$; Atg5 $5^{f / f f}$ ), referred to as WT. Tamoxifen (T5648, Sigma Aldrich) injection (i.p.50 mg/kg) was done daily for 5 consequent days 1 week prior to surgical procedure and Cre- 
expression was confirmed through PCR for genomic DNA using primers encompassing the floxed

524 region, by the appearance of a 125 -bp band.

526 Corneal cauterization lymphangiogenesis assay: The cauterization assay was carried out as previously described $[53,54]$. Eight week old female and male mice, after anaesthetization with intraperitoneal injection of ketamine hydrochloride (100 mg/kg body weight, Thea), xylazine (10 mg/kg body weight, VMD) and the local anesthetic (Unicaïne 0.4\%; Thea Pharma, Wetteren, Belgium), were cauterized in the cornea using an ophthalmic cautery (Optemp II V; Alcon Surgical, Fort Worth, TX). Intraperitoneal (i.p.) injections were done daily with $400 \mu \mathrm{l}$ of a $0.5 \mathrm{M}$ sodium acetate (AC), $50 \mathrm{mg} / \mathrm{kg}$ chloroquine (CQ) or $30 \mathrm{mg} / \mathrm{kg}$ etomoxir (Eto) solution while PBS was injected as a vehicle control, starting the day after corneal cauterization. Mice were euthanized by cervical dislocation nine days post-injury, eyes were

534 removed and corneas dissected for histological analysis. Corneas were fixed in $70 \%$ ethanol for $1 \mathrm{~h}$ at 535 room temperature, blocked in 3\% BSA-3\% Gloria milk (Nestlé) for another hour, washed with PBS and 536 incubated overnight with anti-LYVE1 (R\&D systems, AF2125), anti-CD31 (Pharmingen, 553370) diluted 537 1/200 in PBS-1\% BSA. After $1 \mathrm{~h}$ washing in PBS at room temperature corneas were subsequently 538 incubated with anti-goat/AF488 (Molecular Probes, A21222) and goat anti-rat/AF546 (Molecular 539 Probes, A11081) diluted $1 / 200$ in PBS-1\% BSA for $2 \mathrm{~h}$. Corneas were washed in PBS for $1 \mathrm{~h}$, flat-mounted 540 on a microscope slide with Vecta-shield mounting medium (Vector Laboratories) and imaged using a $541 \quad$ Leica DMI6000.

543 Isolation of murine LEC: Murine endothelial cells were isolated from lungs after treatment with 544 collagenase $A$ and selected in a magnetic field after the cultures were incubated with magnetic beads 545 (11533D CELLection, Invitrogen) coated with anti-mouse CD102 antibody (553326 BD Biosciences) for 546 the first selection. Endothelial cells were then released from beads and reselected with magnetic beads 547 conjugated with anti-mouse Podoplanin anti-PDPN (8.1.1) (BioLegend) in order to obtain CD102+ and 548 Podoplanin+ cells. Cells were cultured in DMEM/F12 supplemented with $20 \%$ FCS, $100 \mathrm{u} / \mathrm{ml}$ penicillin, 
550 from WT and LEC-ATG5 $\%$ mice were started simultaneously.

552 Lipidomic analysis: Cells were trypsinized, washed three times with cold DPBS and cell pellets were re553 suspended in $0.8 \mathrm{ml}$ DPBS. Lipid extraction and multiple reaction monitoring (MRM)-based 554 phospholipid (semi)quantification analysis was performed as previously described in [55]. In brief, 0.7 $555 \mathrm{ml}$ of homogenized cells were mixed with $0.9 \mathrm{ml} \mathrm{MeOH}: \mathrm{HCl}(1 \mathrm{M})(8: 1), 0.8 \mathrm{ml} \mathrm{CHCl} 3$ and $200 \mu \mathrm{g}$ per $556 \mathrm{ml}$ of the antioxidant 2,6-di-tert-butyl-4-methylphenol (Sigma). The organic fractions were evaporated 557 under vacuum using a Savant Speedvac spd111v (Thermo Fisher Scientific) at room temperature and 558 the remaining lipid pellet was stored at $-20^{\circ} \mathrm{C}$ under argon. Before mass spectrometry analysis, lipid 559 pellets were reconstituted in running solution $\left(\mathrm{CH}_{3} \mathrm{OH}: \mathrm{CHCl}_{3}: \mathrm{NH}_{4} \mathrm{OH} ; 90: 10: 1.25 ; \mathrm{v} / \mathrm{v} / \mathrm{v}\right)$. The lipid 560 standards phosphatidylcholine (PC)25:0, PC43:6, sphingomyelin (SM)30:1, phosphatidylethanolamine 561 (PE)25:0, PE43:6, phosphatidylinositol (PI)25:0, PI31:1, PI43:6, phosphatidylserine (PS)25:0, PS31:1 and 562 PS37:4 (Avanti Polar Lipids) were added based on the amount of DNA of the original sample. 563 Phospholipids were analyzed by electrospray ionization tandem mass spectrometry (ESI-MS/MS) on a 564 hybrid quadrupole linear ion trap mass spectrometer (4000 QTRAP system, AB SCIEX) equipped with a 565 TriVersa NanoMate robotic nanosource (Advion Biosciences) for automated sample injection and 566 spraying as previously described [55]. Phospholipid profiling was executed by (positive or negative) 567 precursor ion or neutral loss scanning at a collision energy of $50 \mathrm{eV}$ or $45 \mathrm{eV}, 35 \mathrm{eV},-35 \mathrm{eV}$ and $-60 \mathrm{eV}$ 568 for precursor 184 (SM or PC), neutral loss $141(\mathrm{PE})$, neutral loss 87 (PS) and precursor 241 (PI), 569 respectively. Phospholipid quantification was performed by MRM, the transitions being based on the 570 neutral losses or the typical product ions as described above. Typically, a 3-min period of signal 571 averaging was used for each spectrum. The data were corrected for carbon isotope effects and chain 572 length, and analyzed using in-house-developed software (RALP). As a background, the intensities of 573 species detected in the 'internal standards only' spectra were considered after being divided by the 574 ion suppression factor of each sample. The ion suppression factor was calculated for each sample 
575

576

577

578

separately by dividing the intensity of the standards in the 'internal standards only' spectrum by the intensity of the standards in the sample spectrum. Only the phospholipid species displaying an intensity of at least 5 times the blank value were taken into account. To quantify the total amount of phospholipids in a phospholipid class, the abundances of individually measured species within the phospholipid class were totalled. Data were normalized on the basis of DNA amount.)

Electron microscopy: Transmission electron microscopy (TEM) was performed on a JEOL JEM1400 (JEOL Europe BV, Zaventem, Belgium) (VIB Bio Imaging Core, Leuven Platform). For TEM observations samples were fixed for $24 \mathrm{~h}$ with $2.5 \%$ glutaraldehyde at $\mathrm{pH} 7.3$, buffered with $0.05 \mathrm{M}$ sodium cacodylate. Prior to embedding in Agar 100 Resin (Agar Scientific, Stansted, UK) the material was post fixed in $2 \% \mathrm{OsO}$ (buffered with $0.05 \mathrm{M}$ sodium cacodylate, $\mathrm{pH} 7.3$ ) and dehydrated in a graded acetone series. Semi-thin $( \pm 1 \mathrm{~mm})$ sections were cut with a Reichert Jung Ultracut E microtome and stained with $0.1 \%$ thionin $-0.1 \%$ methylene blue. The ultra-thin $( \pm 70 \mathrm{~nm})$ sections, on copper grids, were stained with uranyl acetate and lead citrate.

Statistical analysis: All data are represented as mean \pm SEM. Statistical significance between two groups was determined by standard two-tailed t-test with F-testing or one sample t-test. Unless otherwise indicated, statistical significance between multiple groups was determined by one-way ANOVA to ensure comparable variance, then individual comparisons performed by Tukey's post-hoc test (Prism v8.0f, GraphPad). $* / \$ / \#$ represents a $p$-value $<0.05, * * / \# \# / \$ \$<0.01, * * * / \# \# \# / \$ \$ / \S \S \S p$ $<0.001$, where a $p$-value $<0.05$ is considered significant.

\section{Acknowledgments}

P.A. is supported by grants from the Flemish Research Foundation (FWO-Vlaanderen; G076617N, G049817N, G070115N), the EOS consortium (30837538) and Stichting tegen Kanker (FAFF/2018/1252). D.H. is the recipient of an FWO Doctoral Fellowship from the Flemish Research 
601 Foundation (FWO-Vlaanderen, 1186019N), Belgium. M.B. is supported by the 'Fonds voor

602 Wetenschappelijk Onderzoek' (FWO). P.C. is supported by Methusalem funding by the Flemish 603 government, and by an ERC Advanced Research Grant (EU-ERC269073).

604

605

Author Contributions

606

O.M., D.H., T.D., H.M., M.S., S.M., M.G.,M.G.C., J.V., M.B., S.B., B.G., M.D., J.S., S.V., M.S., P.C., A.N.,

and P.A. contributed to the research and data analysis. O.M. and P.A. designed the experiments. O.M.,

D.H., T.D., M.G., M.G.C. contributed to experiments execution, analysis, data interpretation, and

609 advice (with input of other co-authors). O.M. and P.A. wrote the article. P.A. conceptualized and

610 directed the study. All authors discussed the results and commented on the manuscript.

\section{Declaration of Interest}

612 P.A. is named as inventor on patent applications claiming subject matter related to the results

613 described in this paper. The other authors declare no competing interests.

614 Antibodies, Reagents and other Recourses

\begin{tabular}{|c|c|c|}
\hline REAGENT or RESOURCE & SOURCE & IDENTIFIER \\
\hline \multicolumn{3}{|l|}{ Antibodies } \\
\hline Anti Goat IgG, HRP-linked & Thermo Fisher Scientific & $\begin{array}{l}\text { PA1-28823 } \\
\text { RRID:AB_10986856 }\end{array}$ \\
\hline Anti-Goat IgG, Alexa Fluor 488 & Molecular Probes & $\begin{array}{l}\text { A21222 } \\
\text { RRID: } \\
\text { AB_10373853 }\end{array}$ \\
\hline Anti-Mouse IgG, HRP-linked & Cell Signaling Technology & $\begin{array}{l}\text { 7076V } \\
\text { RRID: AB_330924 }\end{array}$ \\
\hline Anti-Rabbit IgG, HRP-linked & Cell Signaling Technology & $\begin{array}{l}\text { 7074V } \\
\text { RRID:AB_2099233 }\end{array}$ \\
\hline Goat anti-LYVE1 & R\&D systems & $\begin{array}{l}\text { AF2089 } \\
\text { RRID: AB_355144 }\end{array}$ \\
\hline Goat anti-LYVE1 & R\&D systems & $\begin{array}{l}\text { AF2125 } \\
\text { RRID: AB } 2297188\end{array}$ \\
\hline Goat anti-Mouse IgG, Alexa Fluor 647 & Thermo Fisher Scientific & $\begin{array}{l}\text { A21235 } \\
\text { RRID: AB } 2535804\end{array}$ \\
\hline Hamster anti-PDPN & Biolegend & $\begin{array}{l}\text { 127401 } \\
\text { RRID: AB_1089186 }\end{array}$ \\
\hline Mouse anti-CD36 & Abcam & $\begin{array}{l}\text { ab17044 } \\
\text { RRID: } \underline{A B \quad 443600}\end{array}$ \\
\hline Mouse anti-DRP1 & BD Biosciences & $\begin{array}{l}611113 \\
\text { RRID:AB_398424 }\end{array}$ \\
\hline Mouse anti-NR2F2 & Abcam & ab41859 \\
\hline Mouse anti-OPA1 & BD Biosciences & $\begin{array}{l}\text { 612607 } \\
\text { RRID: AB_399889 }\end{array}$ \\
\hline
\end{tabular}




\begin{tabular}{|c|c|c|}
\hline Mouse anti-TOMM20 & BD Biosciences & $\begin{array}{l}\text { BD 612278 } \\
\text { RRID:AB_399595 }\end{array}$ \\
\hline Mouse anti-ULK1 & Abcam & ab56344 \\
\hline Rabbit anti- Phospho-DRP1 (Ser616) & Cell Signaling Technology & $\begin{array}{l}\text { 3455S } \\
\text { RRID:AB_2085352 }\end{array}$ \\
\hline Rabbit anti-acetyl histone H3 (lysine 9) & Cell Signaling Technology & $\begin{array}{l}9671 \\
\text { RRID: AB } 331532\end{array}$ \\
\hline Rabbit anti-ATG5 & Cell Signaling Technology & $\begin{array}{l}\text { 12994S } \\
\text { RRID: AB_2630393 }\end{array}$ \\
\hline Rabbit anti-ATG7 & Cell Signaling Technology & $\begin{array}{l}\text { 8558S } \\
\text { RRID:AB_10831194 }\end{array}$ \\
\hline Rabbit anti-CPT1 & Cell Signaling Technology & $\begin{array}{l}\text { D3B3 } \\
\text { RRID: AB_2797857 }\end{array}$ \\
\hline Rabbit anti-CPT2 & Abcam & ab181114 \\
\hline Rabbit anti-ENOS & BD Biosciences & 610297 \\
\hline Rabbit anti-FABP4 & Cell Signaling Technology & $\begin{array}{l}\text { 2120S } \\
\text { RRID: AB_2102466 }\end{array}$ \\
\hline Rabbit anti-GAPDH & Cell Signaling Technology & $\begin{array}{l}2118 \mathrm{~S} \\
\text { RRID: AB } 561053\end{array}$ \\
\hline Rabbit anti-LC3 & Cell Signaling Technology & $\begin{array}{l}3868 \mathrm{~S} \\
\text { RRID: AB } 2137707\end{array}$ \\
\hline Rabbit anti-p62 & Millipore & $\begin{array}{l}\text { p0067 } \\
\text { RRID: AB_1841064 }\end{array}$ \\
\hline Rabbit anti-pan-acetyl-histone H3 & Active Motif & $\begin{array}{l}39139 \\
\text { RRID: AB_2687871 }\end{array}$ \\
\hline Rabbit anti-PROX1 & Proteintech & $\begin{array}{l}\text { 11067-2 } \\
\text { RRID: } \mathrm{AB} \quad 2268804\end{array}$ \\
\hline Rabbit anti-VEGFR3 & Abcam & ab154079 \\
\hline Rabbit anti-Vinculin & Cell Signaling Technology & $\begin{array}{l}\# 4650 \\
\text { RRID:AB_10559207 }\end{array}$ \\
\hline Rabbit anti- $\beta$-actin & Sigma-Aldrich & $\begin{array}{l}\text { A5441 } \\
\text { RRID: AB } 476744\end{array}$ \\
\hline Rat anti-CD102 & BD Biosciences & $5 5 3 3 2 6 \longdiv { 2 }$ \\
\hline Rat anti-CD31 & Pharmigen & 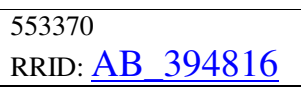 \\
\hline \multicolumn{3}{|l|}{ Chemicals, Peptides, and Recombinant Proteins } \\
\hline$\left[6-{ }^{3} \mathrm{H}\right]$ thymidine & Perkin Elmer & NET355L005MC \\
\hline 2-deoxy-D-glucose & Sigma Aldrich & D-6134 \\
\hline Antimycin A & Sigma-Aldrich & A8674 \\
\hline BMS-303141 (ATP citrate lyase inhibitor) & Sigma-Aldrich & SML0784 \\
\hline Chloroquine & Sigma-Aldrich & C6628 \\
\hline Etomoxir & Sigma-Aldrich & E1905 \\
\hline Glucose & Sigma-Aldrich & 8769 \\
\hline Glutamine & Sigma-Aldrich & G7513 \\
\hline L-Carnitine & Sigma-Aldrich & 8400920025 \\
\hline Methylcellulose & Sigma-Aldrich & M6385 \\
\hline Mitomycin $\mathrm{C}$ & Sigma-Aldrich & M4287 \\
\hline Oligomycin & Sigma-Aldrich & 75351 \\
\hline Palmitic acid N-hydroxysuccinimide ester & Sigma-Aldrich & $14464-31-4$ \\
\hline Palmitic acid- ${ }^{13} \mathrm{C}_{16}$ & Sigma-Aldrich & 605573 \\
\hline PF-04620110 (diglyceride acyltransferase inhibitor 1) & Sigma-Aldrich & PZ0207 \\
\hline PF-06424439 (diglyceride acyltransferase inhibitor 2) & Sigma-Aldrich & PZ0233 \\
\hline Phenylhydrazone (FCCP) & Sanbio & $1528-10$ \\
\hline Propidium iodide & Sigma-Aldrich & $\mathrm{P} 4170$ \\
\hline Saponin & Sigma-Aldrich & S-4521 \\
\hline Sodium acetate & Sigma-Aldrich & S2889 \\
\hline Sodium Pyruvate & Thermo Fisher Scientific & S8636 \\
\hline Tamoxifen & Sigma-Aldrich & T5648 \\
\hline
\end{tabular}




\begin{tabular}{|c|c|c|}
\hline Trichloroacetic acid & Sigma-Aldrich & T6399 \\
\hline VEGF-C human recombinant & Sigma-Aldrich & SRP3184 \\
\hline \multicolumn{3}{|l|}{ Critical Commercial Assays } \\
\hline ORA qPCR Green L mix & HighQu & QPD0105 \\
\hline Reverse transcription kit & Qiagen & 205313 \\
\hline RNeasy Plus mini kit & Qiagen & 74136 \\
\hline \multicolumn{3}{|l|}{ Deposited Data } \\
\hline Raw and analyzed data & This paper & \\
\hline \multicolumn{3}{|l|}{ Experimental Models: Cell Lines } \\
\hline Human dermal lymphatic endothelial cells & Promo Cell & C-12217 \\
\hline \multicolumn{3}{|l|}{ Experimental Models: Organisms/Strains } \\
\hline Mouse: $\operatorname{Atg} 5^{\mathrm{f} / \mathrm{fl}}$ & Kuma et al. 2004 & N/A \\
\hline Mouse: Prox1-cre ${ }^{E R T 2}$ & Bazigou et al. 2011 & N/A \\
\hline \multicolumn{3}{|l|}{ Oligonucleotides } \\
\hline ON-TARGETplus Non-targeting Pool & Dharmacon & D-001810-10-20 \\
\hline SMARTpool: ON-TARGETplus ATG5 siRNA & Dharmacon & L-004374-00-0020 \\
\hline SMARTpool: ON-TARGETplus ATG7 siRNA & Dharmacon & L-020112-00-0050 \\
\hline SMARTpool: ON-TARGETplus DRP1 siRNA & Dharmacon & L-012092-00-0005 \\
\hline SMARTpool: ON-TARGETplus ULK1 siRNA & Dharmacon & L-005049-00-0005 \\
\hline \multicolumn{3}{|l|}{ Recombinant DNA } \\
\hline pBABEpuro GFP-LC3 plasmid & Addgene & \#22405 \\
\hline mCherry-hLC3B-pcDNA3.1 & Addgene & \#40827 \\
\hline \multicolumn{3}{|l|}{ Software and Algorithms } \\
\hline Fiji (Image J) & Open Source & https://fiji.sc \\
\hline FlowJo 8.8.6 software & FlowJo, LLC & $\begin{array}{l}\text { https://www.flowjo } \\
\text {.com }\end{array}$ \\
\hline Image Lab Software & Licor & $\begin{array}{l}\text { https://www.licor.com/bio/ } \\
\text { image- } \\
\text { studio/?gclid=EAIaIQobC } \\
\text { hMIrP3L7_uS6wIVGed3 } \\
\text { Ch1uCw9yEAAYASAAE } \\
\text { gJimPD_BwE }\end{array}$ \\
\hline Prism v8 & Graphpad & $\begin{array}{l}\text { http://www.graphp } \\
\text { ad.com }\end{array}$ \\
\hline Seahorse Wave Desktop Software & Agilent & $\begin{array}{l}\text { https://www.agilent.com/? } \\
\text { gclid=EAIaIQobChMIo5r } \\
\text { Hq- } \\
\text { qS6wIVTe7tCh0YYw7gE } \\
\text { AAYASAAEgJd1vD_Bw } \\
\text { E\&gclsrc=aw.ds }\end{array}$ \\
\hline \multicolumn{3}{|l|}{ Other } \\
\hline 4\% Paraformaldehyde & Thermo Fisher Scientific & J19943K2 \\
\hline 4',6-Diamidino-2-Phenylindole, Dihydrochloride & Thermo Fisher Scientific & D1306 \\
\hline BODIPY 493/503 & Thermo Fisher Scientific & D3922 \\
\hline Bovine serum albumin & Sigma-Aldrich & A6003-25G \\
\hline CellVue ${ }^{\mathrm{TM}}$ Jade & Thermo Fisher Scientific & $88-0876-16$ \\
\hline CellVue ${ }^{\mathrm{TM}}$ NIR780 & Thermo Fisher Scientific & $88-0875-16$ \\
\hline Charcoal stripped serum & Thermo Fischer Scientific & A3382101 \\
\hline Collagen gel (rat tail) & Merck & $08-115$ \\
\hline ECGMV2 & Promo Cell & $\mathrm{C}-22211$ \\
\hline ECGMV2 Supplement Mix & Promo Cell & C-39226 \\
\hline Gelatin from bovine skin & Sigma-Aldrich & G9391 \\
\hline Goat serum & Cell Signaling Technology & $\# 5425$ \\
\hline Hepes & Gibco & 12509079 \\
\hline
\end{tabular}




\begin{tabular}{|c|c|c|}
\hline Ketamine hydrochloride & Dechra & \\
\hline Lipofectamine 2000 & Invitrogen & 16688 \\
\hline Magnetic beads CELLection & Invitrogen & $11533 \mathrm{D}$ \\
\hline MitoSOX ${ }^{\mathrm{TM}}$ Red Mitochondrial Superoxide Indicator & Thermo Fischer Scientific & M36008 \\
\hline Prolong ${ }^{\circledR}$ Gold Antifade Reagent & Thermo Fisher Scientific & P36934 \\
\hline Seahorse XF Assay Medium & Agilent & $103335-100$ \\
\hline Seahorse Xp culture plates & Agilent & $103022-100$ \\
\hline Supplement Mix 2 & Promo Cell & C-39216 \\
\hline Supplement Mix MV2 & Promo Cell & C-39226 \\
\hline TMRM & Thermo Fischer Scientific & T-668 \\
\hline Unicaïne & Thea & 048720 \\
\hline Xylazine & VMD & \\
\hline
\end{tabular}

615

616

617

\section{REFERENCES}

618 1. Alitalo, K., The lymphatic vasculature in disease. Nat Med, 2011. 17(11): p. 1371-80.

$6192 . \quad$ Stacker, S.A., et al., Lymphangiogenesis and lymphatic vessel remodelling in cancer. Nat Rev

$620 \quad$ Cancer, 2014. 14(3): p. 159-72.

621 3. Francois, M., et al., Sox18 induces development of the lymphatic vasculature in mice. Nature,

$622 \quad$ 2008. 456(7222): p. 643-7.

623 4. Srinivasan, R.S., et al., The nuclear hormone receptor Coup-TFIl is required for the initiation and early maintenance of Prox1 expression in lymphatic endothelial cells. Genes Dev, 2010. 24(7): p. 696-707.

5. Srinivasan, R.S., et al., The Prox1-Vegfr3 feedback loop maintains the identity and the number of lymphatic endothelial cell progenitors. Genes Dev, 2014. 28(19): p. 2175-87.

6. Vaahtomeri, K., et al., Lymphangiogenesis guidance by paracrine and pericellular factors. Genes Dev, 2017. 31(16): p. 1615-1634.

7. Johnson, N.C., et al., Lymphatic endothelial cell identity is reversible and its maintenance requires Prox1 activity. Genes Dev, 2008. 22(23): p. 3282-91.

8. Ducoli, L. and M. Detmar, Beyond PROX1: transcriptional, epigenetic, and noncoding RNA regulation of lymphatic identity and function. Dev Cell, 2021. 56(4): p. 406-426.

9. Wong, B.W., et al., The role of fatty acid B-oxidation in lymphangiogenesis. Nature, 2016. 542: p. 49.

10. Schaaf, M.B., et al., Autophagy in endothelial cells and tumor angiogenesis. Cell Death Differ, 2019. 26(4): p. 665-679.

11. Nussenzweig, S.C., S. Verma, and T. Finkel, The role of autophagy in vascular biology. Circ Res, 2015. 116(3): p. 480-8.

12. Abdrakhmanov, A., V. Gogvadze, and B. Zhivotovsky, To Eat or to Die: Deciphering Selective Forms of Autophagy. Trends in biochemical sciences, 2020. 45(4): p. 347-364.

13. Jarc, E. and T. Petan, Lipid Droplets and the Management of Cellular Stress. Yale J Biol Med, 2019. 92(3): p. 435-452.

14. du Toit, A., et al., Measuring autophagosome flux. Autophagy, 2018. 14(6): p. 1060-1071.

15. Cao, R., et al., Mouse corneal lymphangiogenesis model. Nature Protocols, 2011. 6(6): p. 817826.

16. Maes, H., et al., Tumor vessel normalization by chloroquine independent of autophagy. Cancer Cell, 2014. 26(2): p. 190-206.

17. Birgisdottir, A.B. and T. Johansen, Autophagy and endocytosis - interconnections and interdependencies. J Cell Sci, 2020. 133(10). 
18. Wigle, J.T., et al., An essential role for Prox1 in the induction of the lymphatic endothelial cell phenotype. The EMBO Journal, 2002. 21(7): p. 1505-1513.

19. Lin, F.J., et al., Direct transcriptional regulation of neuropilin-2 by COUP-TFII modulates multiple steps in murine lymphatic vessel development. J Clin Invest, 2010. 120(5): p. 1694-707.

20. Maes, H., et al., Autophagy: shaping the tumor microenvironment and therapeutic response. Trends in Molecular Medicine, 2013. 19(7): p. 428-446.

21. Esteban-Martínez, L., et al., Programmed mitophagy is essential for the glycolytic switch during cell differentiation. The EMBO Journal, 2017. 36(12): p. 1688-1706.

22. Ubellacker, J.M., et al., Lymph protects metastasizing melanoma cells from ferroptosis. Nature, 2020. 585(7823): p. 113-118.

23. Olzmann, J.A. and P. Carvalho, Dynamics and functions of lipid droplets. Nature Reviews Molecular Cell Biology, 2019. 20(3): p. 137-155.

24. Schott, M.B., et al., Lipid droplet size directs lipolysis and lipophagy catabolism in hepatocytes. J Cell Biol, 2019. 218(10): p. 3320-3335.

25. Ouimet, M., et al., Autophagy regulates cholesterol efflux from macrophage foam cells via Iysosomal acid lipase. Cell Metab, 2011. 13(6): p. 655-67.

26. Bowden, K.L., et al., Lysosomal acid lipase deficiency impairs regulation of ABCA1 gene and formation of high density lipoproteins in cholesteryl ester storage disease. J Biol Chem, 2011. 286(35): p. 30624-35.

27. Harris, C.A., et al., DGAT enzymes are required for triacylglycerol synthesis and lipid droplets in adipocytes. J Lipid Res, 2011. 52(4): p. 657-67.

28. Chitraju, C., T.C. Walther, and R.V. Farese, Jr., The triglyceride synthesis enzymes DGAT1 and DGAT2 have distinct and overlapping functions in adipocytes. J Lipid Res, 2019. 60(6): p. 11121120.

29. McLaren, D.G., et al., DGAT2 Inhibition Alters Aspects of Triglyceride Metabolism in Rodents but Not in Non-human Primates. Cell Metab, 2018. 27(6): p. 1236-1248 e6.

30. Singh, R., et al., Autophagy regulates lipid metabolism. Nature, 2009. 458(7242): p. $1131-5$.

31. Diebold, L.P., et al., Mitochondrial complex III is necessary for endothelial cell proliferation during angiogenesis. Nat Metab, 2019. 1(1): p. 158-171.

32. Yao, C.H., et al., Identifying off-target effects of etomoxir reveals that carnitine palmitoyltransferase $I$ is essential for cancer cell proliferation independent of beta-oxidation. PLoS Biol, 2018. 16(3): p. e2003782.

33. Kalucka, J., et al., Quiescent Endothelial Cells Upregulate Fatty Acid beta-Oxidation for Vasculoprotection via Redox Homeostasis. Cell Metab, 2018. 28(6): p. 881-894 e13.

34. Siragusa, M., et al., Nitric oxide maintains endothelial redox homeostasis through PKM2 inhibition. EMBO J, 2019. 38(17): p. e100938.

35. Giacomello, M., et al., The cell biology of mitochondrial membrane dynamics. 2020. 21(4): p. 204-224.

36. Yu, S.B. and G. Pekkurnaz, Mechanisms Orchestrating Mitochondrial Dynamics for Energy Homeostasis. J Mol Biol, 2018. 430(21): p. 3922-3941.

37. McDonnell, E., et al., Lipids Reprogram Metabolism to Become a Major Carbon Source for Histone Acetylation. Cell Rep, 2016. 17(6): p. 1463-1472.

38. Arduini, A. and V. Zammit, Acetate transport into mitochondria does not require a carnitine shuttle mechanism. Magn Reson Med, 2017. 77(1): p. 11.

39. Minami, S., et al., Lipophagy maintains energy homeostasis in the kidney proximal tubule during prolonged starvation. 2017. 13(10): p. 1629-1647.

40. Nguyen, T.B., et al., DGAT1-Dependent Lipid Droplet Biogenesis Protects Mitochondrial Function during Starvation-Induced Autophagy. Dev Cell, 2017. 42(1): p. 9-21 e5.

41. Rambold, A.S., S. Cohen, and J. Lippincott-Schwartz, Fatty acid trafficking in starved cells: regulation by lipid droplet lipolysis, autophagy, and mitochondrial fusion dynamics. Dev Cell, 2015. 32(6): p. 678-92. 
42. Benador, I.Y., et al., Mitochondria Bound to Lipid Droplets Have Unique Bioenergetics, Composition, and Dynamics that Support Lipid Droplet Expansion. Cell Metab, 2018. 27(4): p. 869-885 e6.

43. Pernas, L., et al., Mitochondria Restrict Growth of the Intracellular Parasite Toxoplasma gondii by Limiting Its Uptake of Fatty Acids. Cell Metab, 2018. 27(4): p. 886-897 e4.

44. $\mathrm{Xu}, \mathrm{L}$., et al., Ketogenic essential amino acids replacement diet ameliorated hepatosteatosis with altering autophagy-associated molecules. Biochim Biophys Acta, 2013. 1832(10): p. 160512.

45. Galluzzi, L., et al., Metabolic control of autophagy. Cell, 2014. 159(6): p. 1263-76.

46. García-Caballero, M., et al., Role and therapeutic potential of dietary ketone bodies in lymph vessel growth. Nature Metabolism, 2019. 1(7): p. 666-675.

47. Saito, T., et al., Autophagy regulates lipid metabolism through selective turnover of NCoR1. Nat Commun, 2019. 10(1): p. 1567.

48. Korff, T., T. Krauss, and H.G. Augustin, Three-dimensional spheroidal culture of cytotrophoblast cells mimics the phenotype and differentiation of cytotrophoblasts from normal and preeclamptic pregnancies. Experimental Cell Research, 2004. 297(2): p. 415-423.

49. Qiu, B. and M.C. Simon, BODIPY 493/503 Staining of Neutral Lipid Droplets for Microscopy and Quantification by Flow Cytometry. Bio Protoc, 2016. 6(17).

50. Schoors, S., et al., Fatty acid carbon is essential for dNTP synthesis in endothelial cells. Nature, 2015. 520(7546): p. 192-197.

51. Bazigou, E., et al., Genes regulating lymphangiogenesis control venous valve formation and maintenance in mice. The Journal of clinical investigation, 2011. 121(8): p. 2984-2992.

52. Kuma, A., et al., The role of autophagy during the early neonatal starvation period. Nature, 2004. 432(7020): p. 1032-1036.

53. Blacher, S., et al., Additional parameters for the morphometry of angiogenesis and lymphangiogenesis in corneal flat mounts. Experimental Eye Research, 2009. 89(2): p. 274-276.

54. Detry, B., et al., Sunitinib Inhibits Inflammatory Corneal Lymphangiogenesis. Investigative Ophthalmology \& Visual Science, 2013. 54(5): p. 3082-3093.

55. Marien, E., et al., Non-small cell lung cancer is characterized by dramatic changes in phospholipid profiles. Int J Cancer, 2015. 137(7): p. 1539-48. 


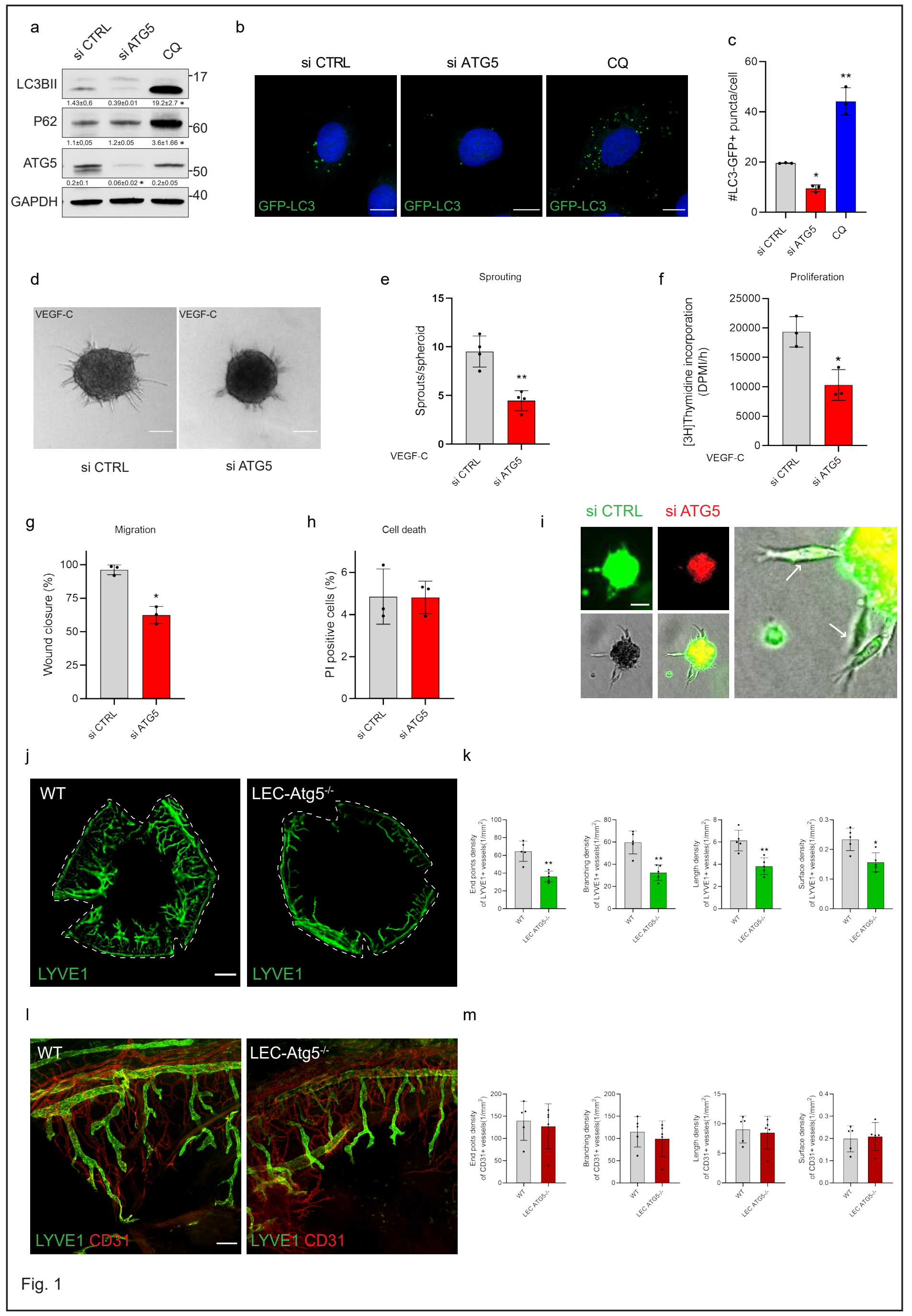




\section{Fig. 1 | Autophagy is essential for lymphatic endothelial cell homeostasis.}

a) Representative blots for indicated proteins of si CTRL, si ATG5 and chloroquine (CQ $25 \mu \mathrm{M}, 48 \mathrm{~h}$ ) treated LEC, with GAPDH as loading control. Densitometric quantification indicated beneath the blots. Mean $\pm \mathrm{SEM}, \mathrm{N}=3$ analyzed using one-way ANOVA, ${ }^{*} p<0.05$ vs si CTRL. b,c) Representative immunofluorescent images (B) and quantification (C) of si CTRL, si ATG5 and CQ-treated LEC after GFPLC3 transfection and DAPI staining. GFP-LC3 puncta accumulation reflects amount of autophagosomes. Mean $\pm \mathrm{SEM}, \mathrm{N}=3$ analyzed by one-way ANOVA, with Tukey's test for multiple comparisons, ${ }^{*} p<0.05$ and ${ }^{*} \mathrm{p}<0.01$ vs si CTRL LEC. Scale bars represent $10 \mu \mathrm{m}$. d) Representative images of si CTRL and si ATG5 LEC spheroids upon stimulation with VEGF-C (100 ng/mL, 48h). Scale bar represents $100 \mu \mathrm{m}$. e) Quantification of sprouts per spheroid. Mean $\pm S E M, N=4$ analyzed using unpaired Student's $t$ test, ${ }^{* *} p$ < 0.01. f) $\left[{ }^{3} \mathrm{H}\right]$ Thymidine incorporation assay into DNA of si CTRL and si ATG5 LEC upon stimulation with VEGF-C. Mean \pm SEM, $N=3$ analyzed using unpaired Student's $t$ test, $* p<0.05$. g) Quantification of scratch wound healing for si CTRL and si ATG5 LEC monolayers in the presence of $500 \mu \mathrm{g} / \mathrm{ml}$ Mitomycin C to block proliferation. Mean $\pm \mathrm{SEM}, \mathrm{N}=3$ analyzed using unpaired Student's $t$ test, $* \mathrm{p}<0.05$. h) Flow cytometry analysis of cell death by propidium iodide (PI) of si CTRL and si ATG5 LEC. Mean \pm SEM, N=3 analyzed using unpaired Student's $t$ test. i) Representative immunofluorescent images of mixed spheroids containing equal amount of fluorescently green labeled si CTRL and red labeled si ATG5 LEC. Images on the left show the single green and red channels as well as brightfield image and merged image. The large image on the right shows sprouts at larger magnification. Arrows denote newly formed sprouts. j) Representative immunofluorescent images of whole corneal mounts of wild type (WT) and LEC-Atg5 knock out mice (LEC-Atg5 ${ }^{-/}$) stained for LYVE1, 8 days post corneal cauterization.

Scale bar represents $1 \mathrm{~mm}$. k) Quantification for the number of end points, number of branch points, average of cumulative length and surface density for LYVE1+ lymphatic vessels. Each data point on graphs represents one cornea. Mean $\pm \mathrm{SEM}, \mathrm{N}=5$ for WT and LEC-Atg5 ${ }^{\%}$ analyzed using unpaired Student's $t$ test, $* p<0.05, * * p<0.01$. I) Representative immunofluorescent images of corneal sections dissected from WT and LEC-Atg5 ${ }^{-/-}$mice stained for LYVE1 and CD31, 8 days post corneal cauterization. Scale bars represent $100 \mu \mathrm{m}$. m) Quantification for the number of end points, number of branch points, average of cumulative length and surface density for CD31+ blood vessels. Mean \pm SEM. $N=5$ for WT and LEC-Atg5 $\%$ analyzed using unpaired Student's $t$ test, $\mathrm{p}=$ not significant. 


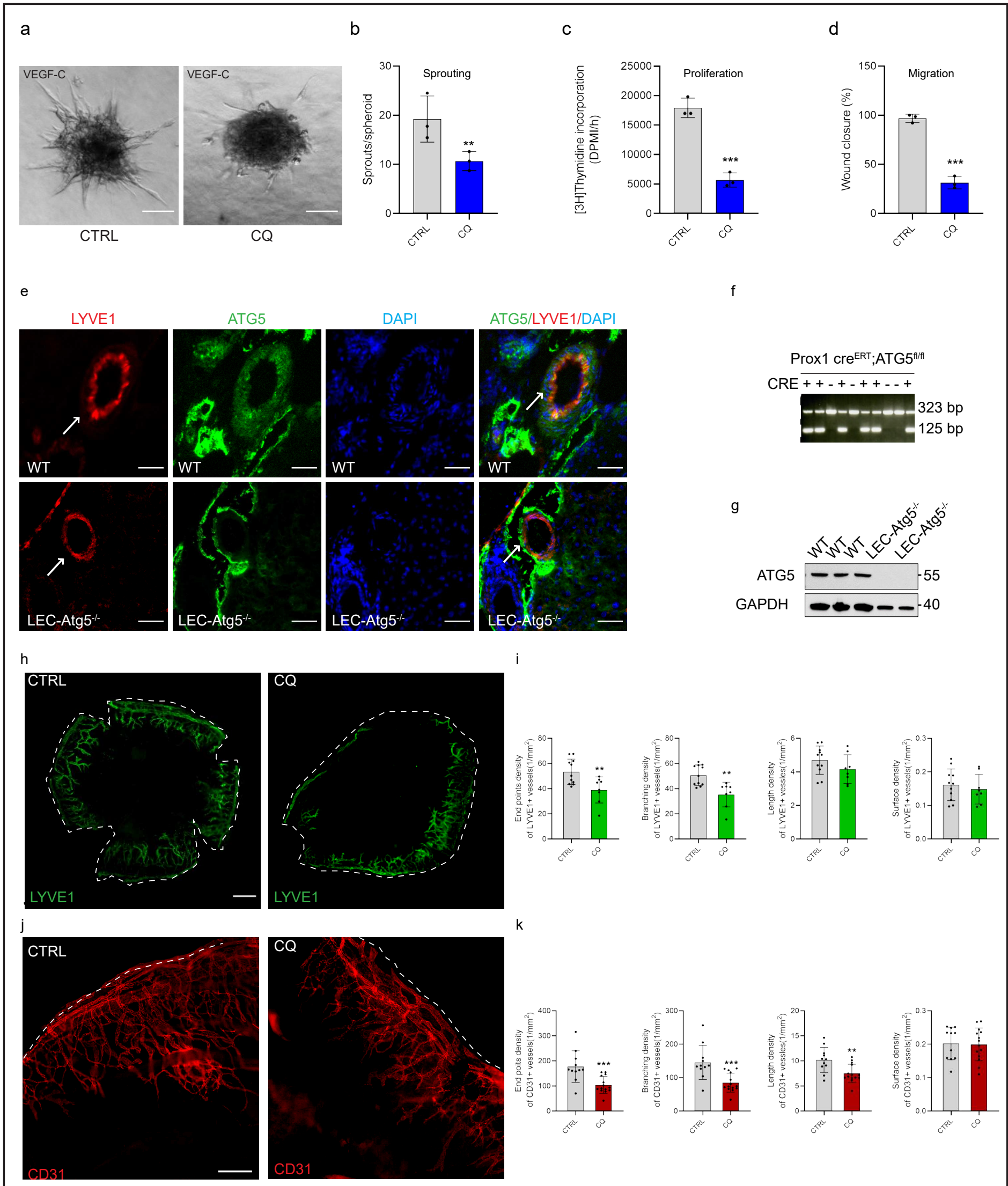




\section{Extended Data Fig.1 | Effects of chloroquine on LEC function and confirmation of LEC-ATG5 knock out mouse (LEC-ATG5 $5^{-/}$).}

a) Representative images of CTRL and CQ- $(25 \mu \mathrm{M}, 48 \mathrm{~h})$ treated LEC spheroids upon stimulation with VEGF-C (100 ng/mL, 48h). Scale bar represents $100 \mu \mathrm{m}$. b) Quantification of sprouts per spheroid. Mean $\pm \mathrm{SEM}, \mathrm{N}=3$ analyzed using unpaired Student's $t$ test $* * p<0.01$. c) $\left[{ }^{3} \mathrm{H}\right]$ Thymidine incorporation assay into DNA of CTRL and CQ-treated LEC upon stimulation with VEGF-C. Mean \pm SEM, N=3 analyzed using unpaired Student's $t$ test, $* * * p<0.001$. d) Quantification of scratch wound healing for CTRL and CQ-treated LEC monolayers. Mean \pm SEM, N=3 analyzed using unpaired Student's $t$ test, $* * * p<0.001$.

e) Dual immunofluorescent staining of LYVE1+ (red) lymphatic vessels and ATG5 (green) from liver sections of WT and LEC-Atg5 ${ }^{-/-}$mice. Note the absence of co-localization between ATG5 and LYVE1+ vessels in the LEC-Atg5\% mice. Scale bar represents $100 \mu \mathrm{m}$. f) Genotyping for the presence of Atg5 floxed (323bp) and excision band upon tamoxifen administration (125bp) in Prox $1 \mathrm{cre}^{\mathrm{ERT}}$;ATG5 ${ }^{\mathrm{fl} / \mathrm{fl}}$ mice upon tamoxifen administration. g) Immunoblot for ATG5 with GAPDH as loading control of LEC isolated from WT and LEC-Atg5 $\%$ mice. h) Representative immunofluorescent images of whole corneal mounts of vehicle treated or CQ-(50 mg/kg daily) treated WT mice, stained for LYVE1, 8 days post corneal cauterization. Scale bar represents $1 \mathrm{~mm}$. i) Quantification for the number of end points, number of branch points, average of cumulative length and surface density for LYVE1+ lymphatic vessels. Each data point on graphs represents one cornea. Mean $\pm \mathrm{SEM}, \mathrm{N}=11$ for CTRL and $\mathrm{N}=8$ for CQ analyzed using unpaired Student's $t$ test, ${ }^{* *} p<0.01$. j) Representative immunofluorescent images of corneal sections dissected from vehicle treated or CQ treated mice stained for CD31, 8 days post corneal cauterization. Scale bar represents $100 \mu \mathrm{m}$. k) Quantification for the number of end points, number of branch points, average of cumulative length and surface density for CD31+ blood vessels. Mean \pm SEM, $\mathrm{N}=11$ for CTRL and N=14 for CQ analyzed using unpaired Student's $t$ test, ${ }^{* *} \mathrm{p}<0.01$ and ${ }^{* * *} \mathrm{p}<0.001$. 
a

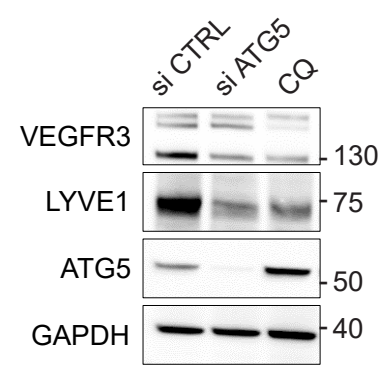

d
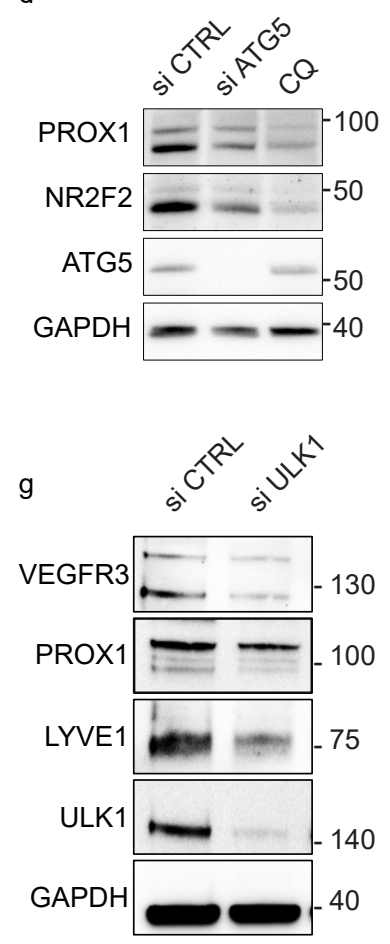

k

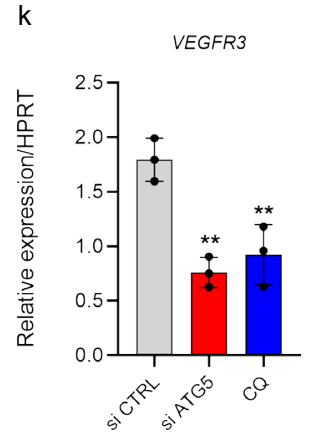

O

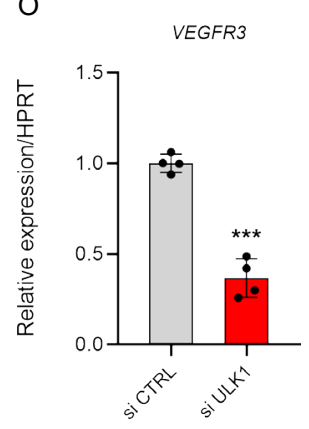

b

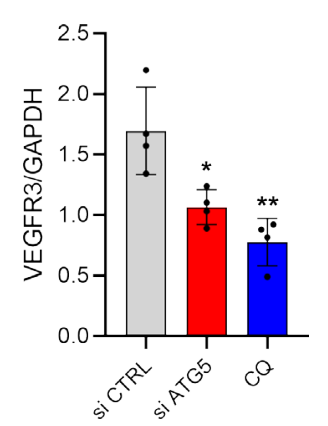

e

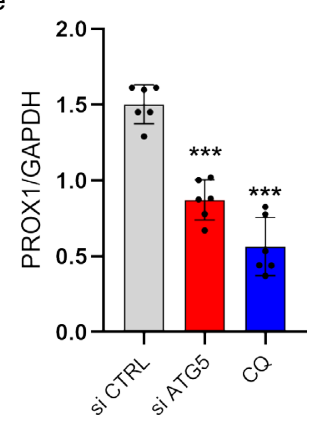

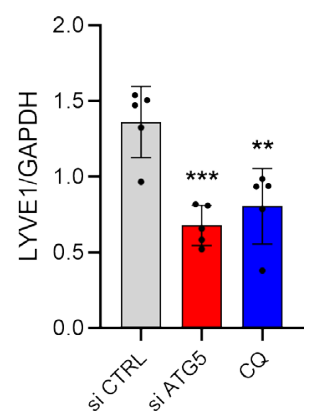

f

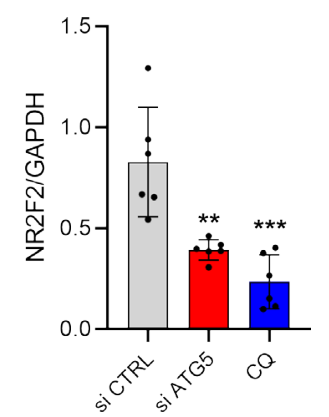

h
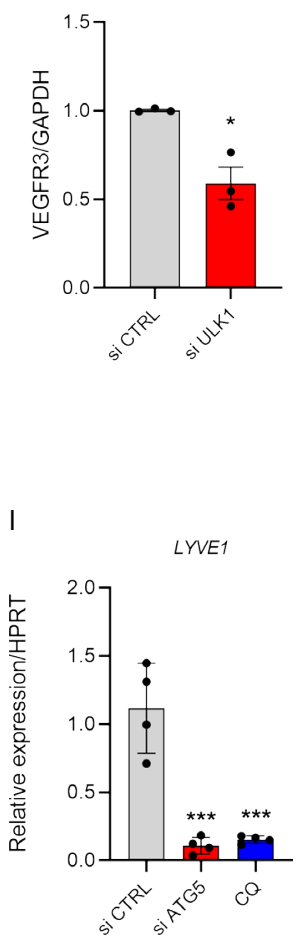

P

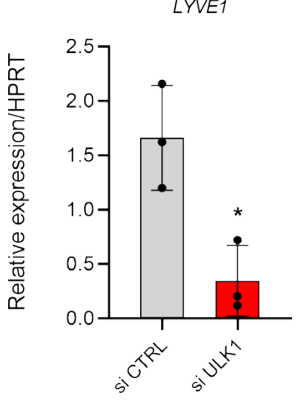

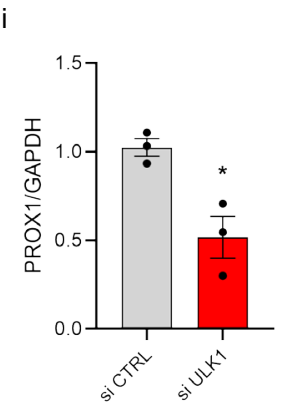
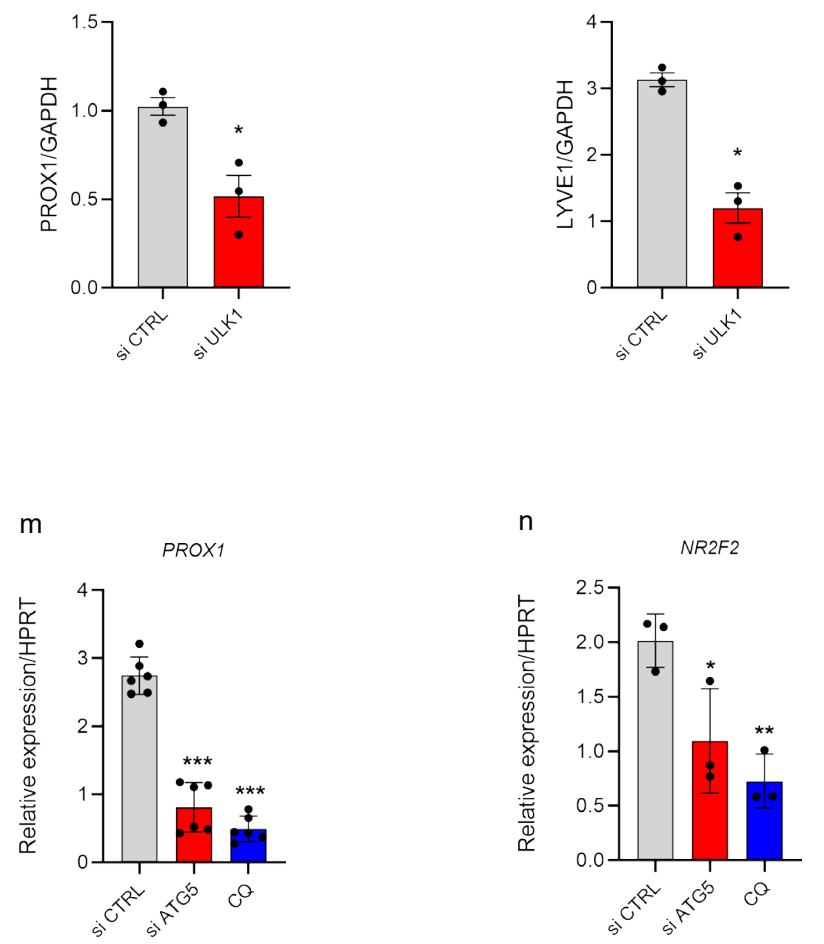

Q
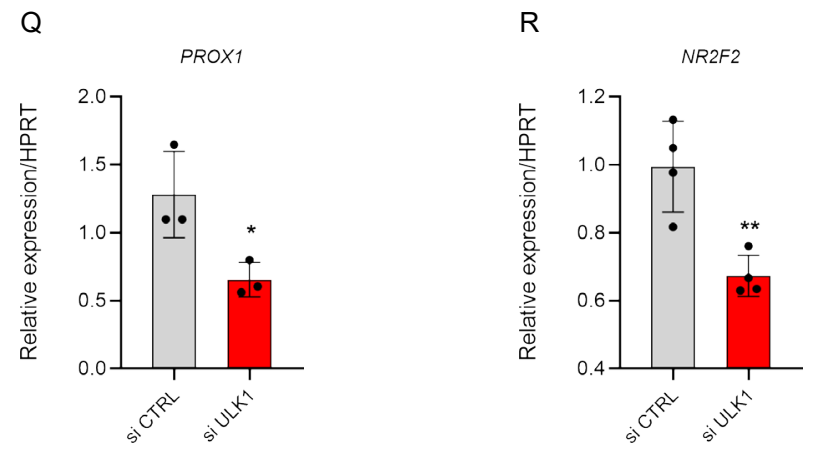

Fig. 2 
Fig. 2 | Genetic inhibition of ATG5 attenuates the expression of crucial lymphatic genes.

a-f) Representative blots $(a, d)$ and corresponding densitometric quantifications $(b, c, e, f)$ for the indicated proteins in si CTRL, si ATG5 or CQ (25 $\mu \mathrm{M}, 48 \mathrm{~h})$ treated LEC after $48 \mathrm{~h}$. Mean $\pm \mathrm{SEM}, \mathrm{N} \geq 4$ analyzed by one-way ANOVA, with Tukey's test for multiple comparisons, ${ }^{*} p<0.05, * * p<0.01,{ }^{* * *} p$ < 0.001 vs si CTRL. g-j) Representative blots (g) and corresponding densitometric quantifications (h-j) for the indicated proteins in si CTRL and si ULK1 LEC after 48h. Mean \pm SEM, N=3 analyzed using unpaired Student's $t$ test, ${ }^{*} p=0.05$. k-n) RT-qPCR analysis of si CTRL, si ATG5 LEC or CQ treated LEC after 48h. mRNA expression of VEGFR3, LYVE1, PROX1 and NR2F2 (relative to HPRT). Mean $\pm \mathrm{SEM}, \mathrm{N} \geq 3$ analyzed by one-way ANOVA, with Tukey's test for multiple comparisons, ${ }^{*} p<0.05, * * p<0.01, * * * p$ < 0.001 vs si CTRL. o-r) RT-qPCR analysis of si CTRL and si ULK1 LEC after 48h. mRNA expression of VEGFR3, LYVE1, PROX1 and NR2F2 (relative to HPRT). Mean $\pm \mathrm{SEM}, \mathrm{N} \geq 3$ analyzed by unpaired Student's $t$ test, $* p<0.05, * * p<0.01, * * * p<0.001$. 


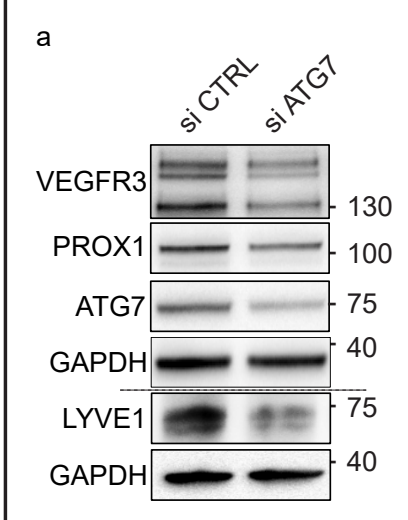

e

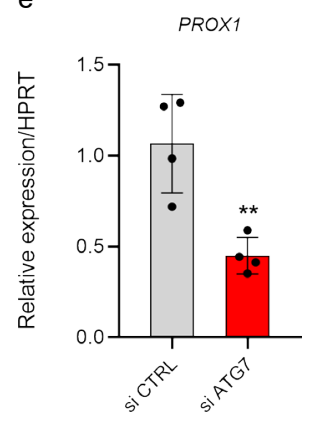

b

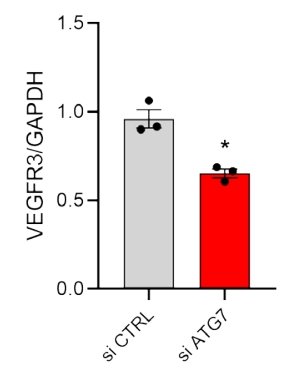

f

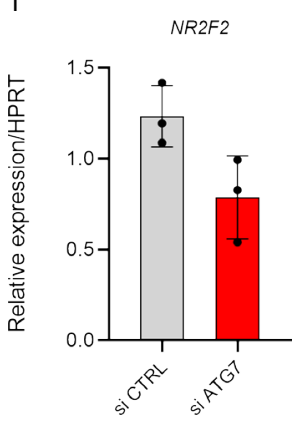

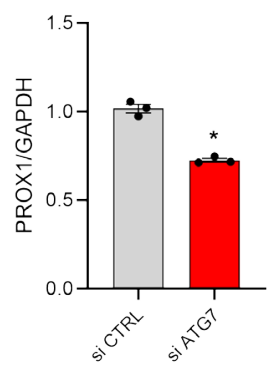

d

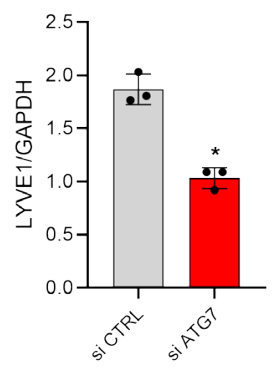

g

VEGFR3

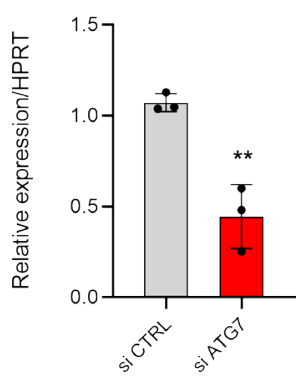

LYVE1

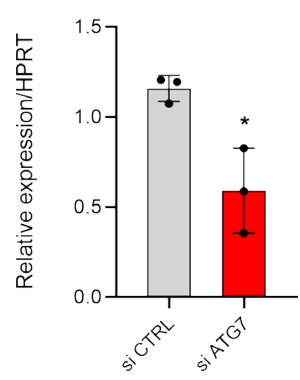

Extended Data Fig.2 | Genetic inhibition of ATG7 attenuates expression of crucial lymphatic genes.

a-d) Representative blots (a) and corresponding densitometric quantifications (b-d) for the indicated proteins in si CTRL and si ATG7 LEC after 48h. Mean \pm SEM, $N \geq 3$ analyzed using unpaired Student's $t$ test, ${ }^{*} p<0.05,{ }^{*} p<0.01$. e-h) RT-qPCR analysis of si CTRL and si ATG7 LEC after 48h. mRNA expression of PROX1, VEGFR3 and LYVE1 (relative to HPRT). Mean $\pm \mathrm{SEM}, \mathrm{N} \geq 3$ analyzed by unpaired Student's $t$ test, $* p<0.05, * * p<0.01$ 


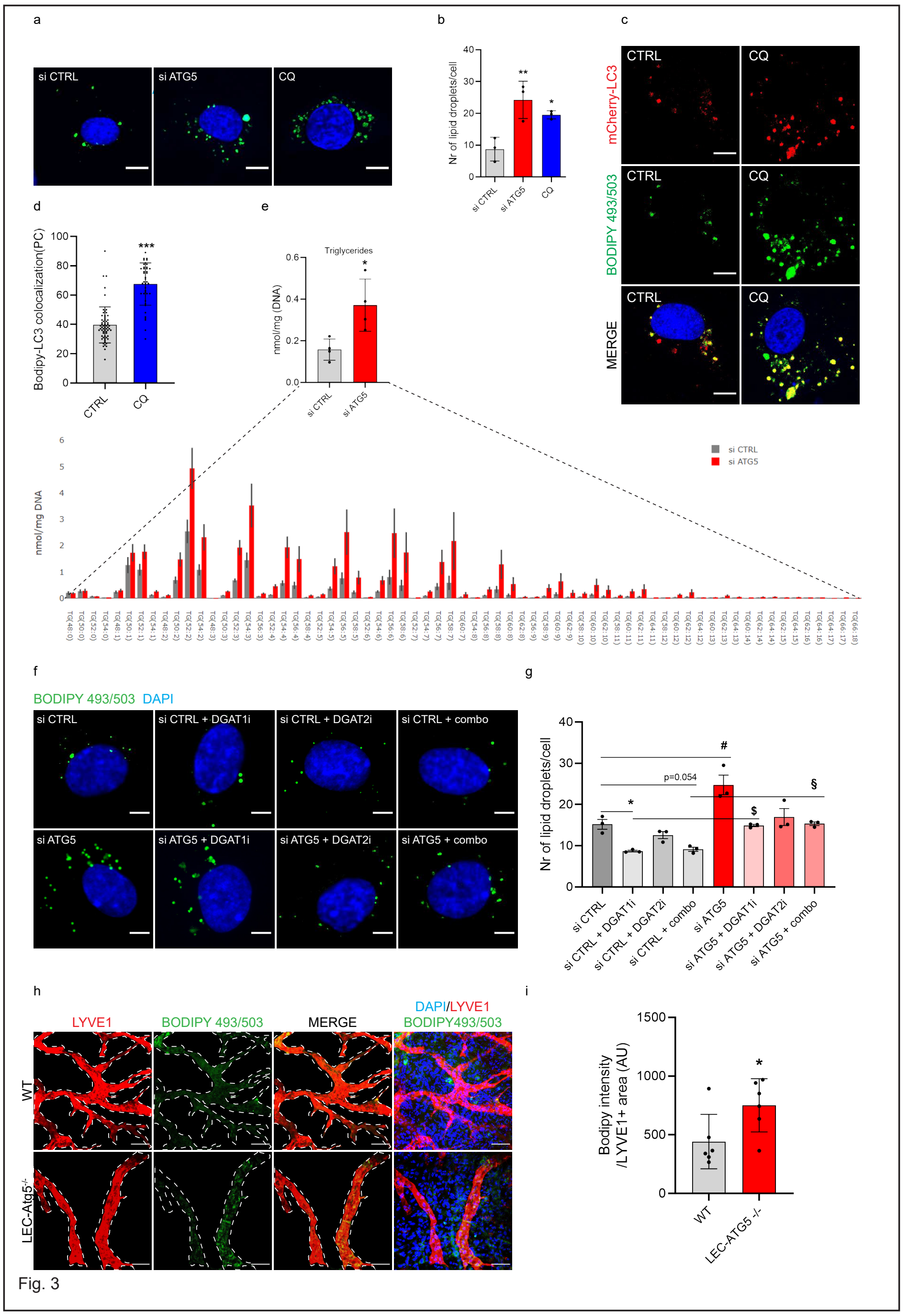


Fig. 3 | Autophagy regulates lipid droplets homeostasis in lymphatic endothelial cells.

a) Representative immunofluorescent images of BODIPY 493/503 staining of lipid droplets in si CTRL, si ATG5 and CQ $(25 \mu \mathrm{M}, 48 \mathrm{~h})$ treated LEC. Nuclei are stained with DAPI. Scale bars represent $10 \mu \mathrm{m}$. b) Quantification of lipid droplet number per cell are shown. Mean $\pm \mathrm{SEM}, \mathrm{N}=3$ analyzed by one-way ANOVA, with Tukey's test for multiple comparisons, ${ }^{*} p<0.05, * * p<0.01$ vs si CTRL. c) Representative immunofluorescent images of BODIPY $493 / 503$ staining of lipid droplets in untreated (CTRL) or CQ treated LEC transfected with a mCherry-LC3 plasmid for $24 \mathrm{~h}$. Nuclei are stained with DAPI. Scale bars represent $10 \mu \mathrm{m}$. d) Co-localization of BODIPY 493/503 (green) and LC3 (red) in untreated (CTRL) or CQ treated LEC (PC= Pearson coefficient). Mean $\pm \mathrm{SEM}, \mathrm{N}=3$ analyzed by unpaired Student's $t$ test, $* * * p<0.001$. e) Triglycerides bar plots calculated as an average of the lipid subspecies analyzed across si CTRL and si ATG5 LEC. Mean \pm SEM, $N=4$ analyzed by unpaired Student's $t$ test, $* p<0.05$. f) Representative immunofluorescent images of DAPI (nuclei) and BODIPY 493/503 staining of lipid droplets in si CTRL, si ATG5 LEC treated with DGAT1 or DGAT2 inhibitors (20 $\mu \mathrm{M})$ or their combination (combo) (48h). Scale bars represent $10 \mu \mathrm{m}$. g) Quantification of lipid droplet number per cell is shown. Mean $\pm \mathrm{SEM}, \mathrm{N}=3$ analyzed with two-way ANOVA with Sidak test for multiple comparisons, ${ }^{*} \mathrm{p}<0.05$ vs si $C T R L, " \# p 0.05$ vs si $C T R L, \quad{ }^{\$} / \$ p<0.05$ vs corresponding control. h) Representative immunofluorescent images of corneal sections dissected from WT and LEC-Atg5 $\%$ mice stained for BODIPY 493/503 and LYVE1. Nuclei are stained with DAPI. Scale bar represents $100 \mu \mathrm{m}$. i) Quantification of BODIPY 493/503 (green) of the LYVE1 positive area (red, representative of lymphatic vessel area) are shown. Mean $\pm \mathrm{SEM}, \mathrm{N}=6$ analyzed using unpaired Student's $t$ test, ${ }^{*} \mathrm{p}<0.05$. 
a

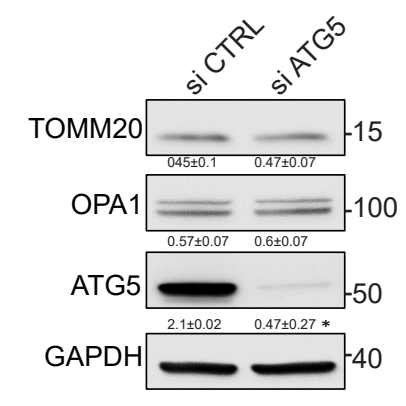

d

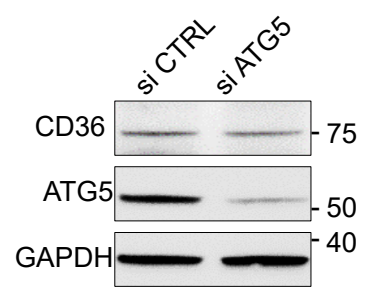

b

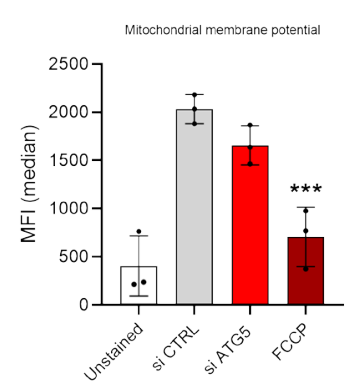

e

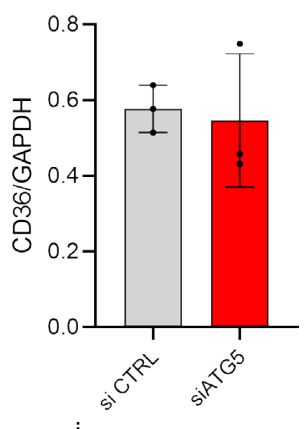

c

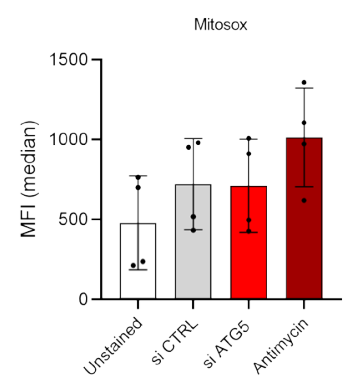

g

h
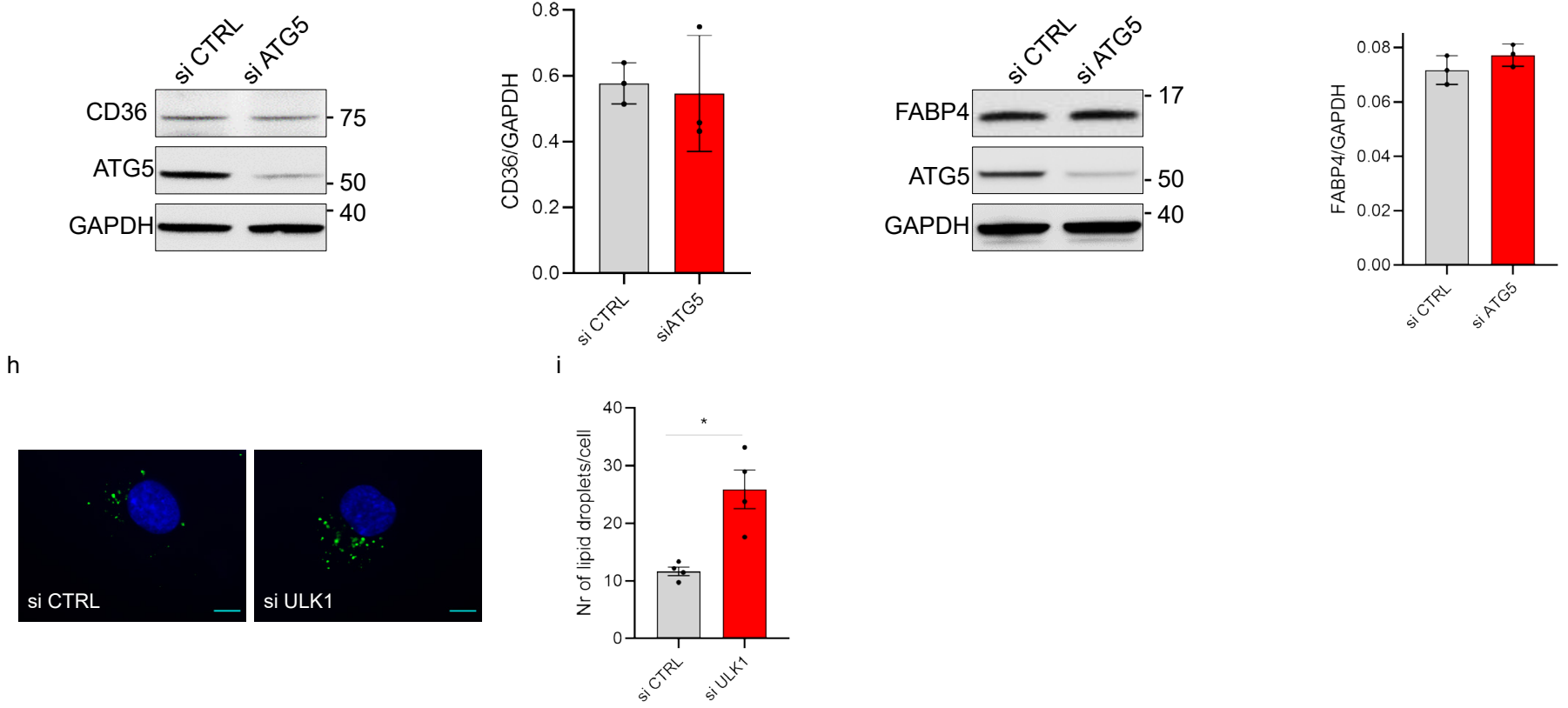

si ATG5

$C Q$
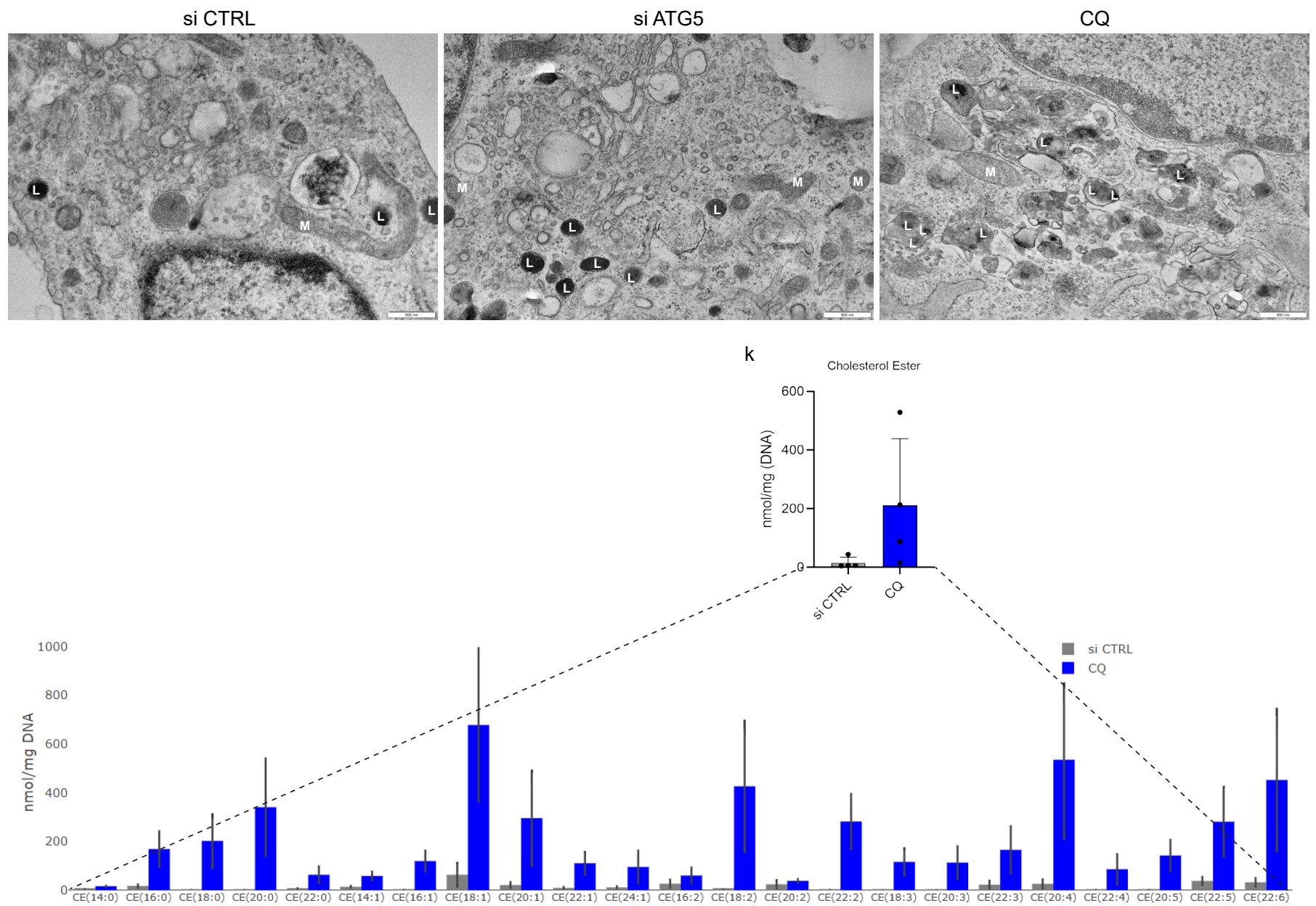

Extended Data Fig. 3 


\section{Extended Data Fig.3 | Effect of genetic or pharmacological inhibition of autophagy on mitochondrial}

protein turnover, mitochondrial ROS, mitochondrial membrane potential and lipid droplets.

(a) Representative blots for the indicated proteins in si CTRL, si ATG5 LEC after 48h. Densitometric quantification indicated beneath the blots. Mean $\pm \mathrm{SEM}, \mathrm{N}=3$ analyzed using unpaired Student's $t$ test, $*_{p}<0.05$. (b-c) Flow cytometry analysis for mitochondrial membrane potential by TMRM (b) and mitochondrial ROS (c) by Mitosox in si CTRL and si ATG5 LEC after 48h. FCCP and Antimycin were used as positive controls in (b) and (c), respectively. Mean \pm SEM, $N=4$ analyzed by one-way ANOVA, with Tukey's test for multiple comparisons, ${ }^{* * *} p<0.001$ compared to si CTRL. (d-g) Representative blots $(d, f)$ and corresponding densitometric quantifications $(e, g)$ for the indicated proteins in si CTRL and si ATG5 LEC after $48 \mathrm{~h}$. Mean \pm SEM, N=3 analyzed using unpaired Student's $t$ test. h) Representative immunofluorescent images of BODIPY 493/503 staining of lipid droplets in si CTRL and si ULK1 LEC. Scale bars represent $10 \mu \mathrm{m}$. i) Quantification of lipid droplet number per cell. Mean $\pm \mathrm{SEM}, \mathrm{N}=4$ analyzed using unpaired Student's $t$ test, ${ }^{*} p<0.05$. j) Representative electron microscopy images revealing increased electron-dense lipid droplet accumulation, labeled with ' $\mathrm{L}$ ' and mitochondria ' $\mathrm{M}$ ' in si CTRL, si ATG5 or CQ-treated LEC (48h). k) Cholesterol ester (CE) bar plots calculated as an average

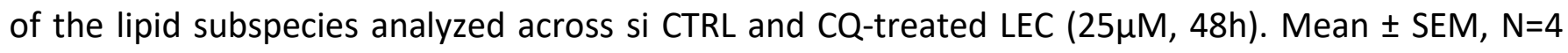
analyzed by unpaired Student's $t$ test. 


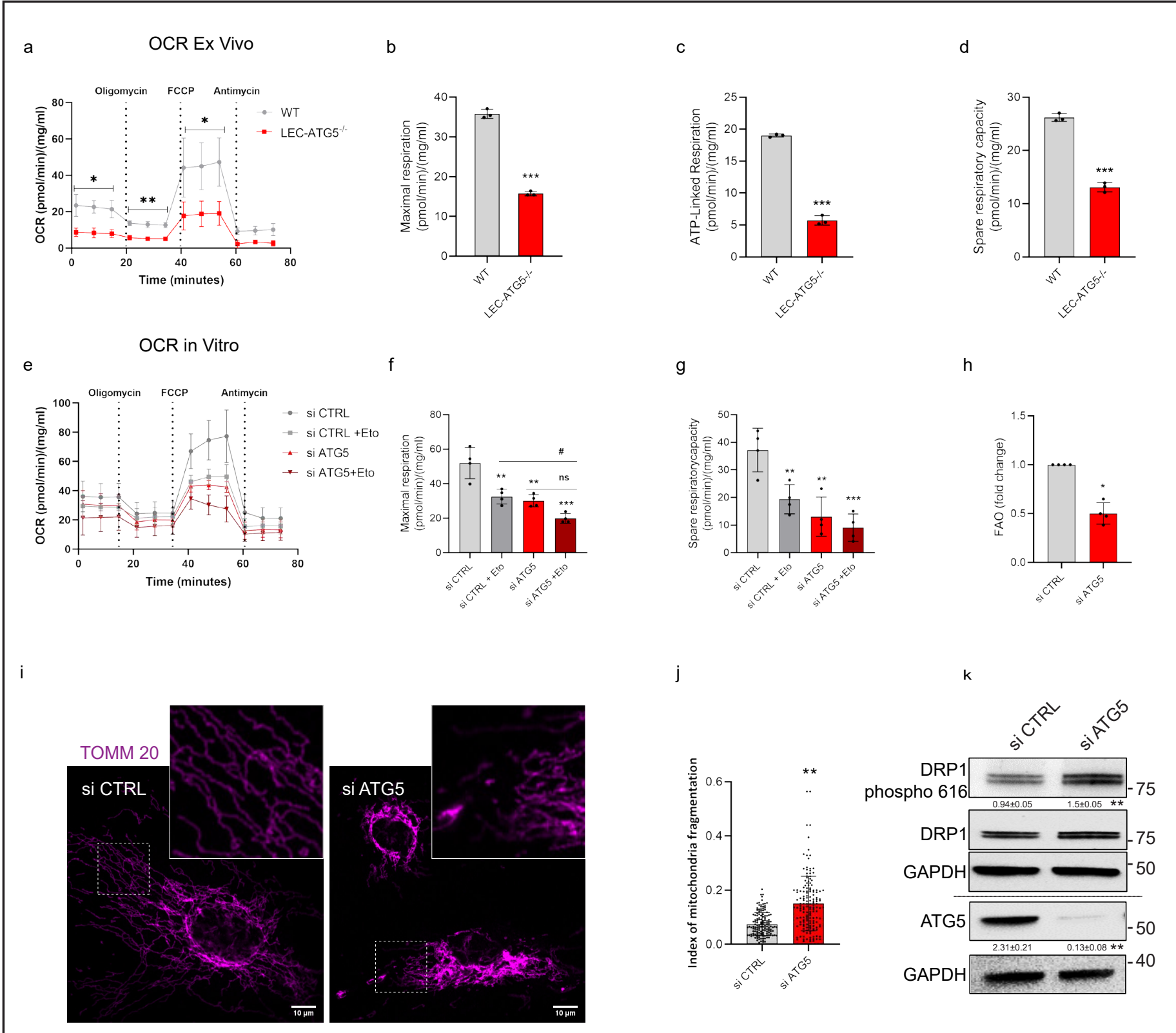

Fig. 4 
Fig. 4 | Functional LEC-autophagy is essential for mitochondrial morphology and fatty acid oxidation. a) Oxygen consumption rate (OCR) of LEC isolated from WT or LEC-Atg $5 \%$ determined at baseline, after oligomycin, phenylhydrazone (FCCP) and antimycin treatment, analyzed via the Seahorse XFp Extracellular flux analyzer. Mean $\pm \mathrm{SEM}, \mathrm{N}=4$ analyzed using unpaired Student's $t$ test, $* \mathrm{p}<0.05,{ }^{*} \mathrm{p}$ < 0.01. b) Maximal respiration c) ATP-linked respiration and d) Spare respiratory capacity from A. Mean $\pm \mathrm{SEM}, \mathrm{N}=3$ using unpaired Student's $t$ test, $* * * p<0.001$. e) OCR of si CTRL or si ATG5 LEC measured in ß-oxidation assay medium. Etomoxir (Eto, $40 \mu \mathrm{M}$ ) was added $15 \mathrm{~min}$ prior to baseline measurement in ß-oxidation assay medium. f) Maximal respiration and g) Spare respiratory capacity from OCR in E. Mean $\pm \mathrm{SEM}, \mathrm{N}=4$ analyzed using a two-way ANOVA with Tukey's test for multiple comparisons, ${ }^{*} \mathrm{p}<$ $0.01, * * * p<0.001$ vs si CTRL and ${ }^{*} p<0.05$ vs si CTRL + Eto. $\mathbf{h}$ ) Fatty acid oxidation assay of si CTRL and si ATG5 LEC supplemented with $2 \mathrm{mCi} / \mathrm{mL}\left[9,10-{ }^{3} \mathrm{H}\right]$-palmitic acid for $18 \mathrm{~h}$. Mean $\pm \mathrm{SEM}, \mathrm{N}=4$ analyzed using one sample Student's $t$ test, $* p<0.05$. i) Representative immunofluorescent images of the mitochondrial protein TOMM20 from si CTRL and si ATG5 LEC. j) Quantification of the mitochondrial fragmentation index. Mean $\pm \mathrm{SEM}, \mathrm{N}=5$ analyzed using unpaired Student's $t$ test, $* * p<0.01$. k) Representative immunoblot of phospho-DRP1 (Ser 616), total DRP1 and GAPDH. Densitometric quantification indicated beneath the blot. Mean $\pm \mathrm{SEM}, \mathrm{N}=3$ using unpaired Student's $t$ test, ${ }^{*} \mathrm{p}<0.05$. 


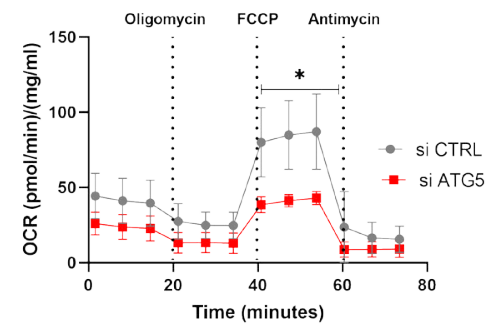

e
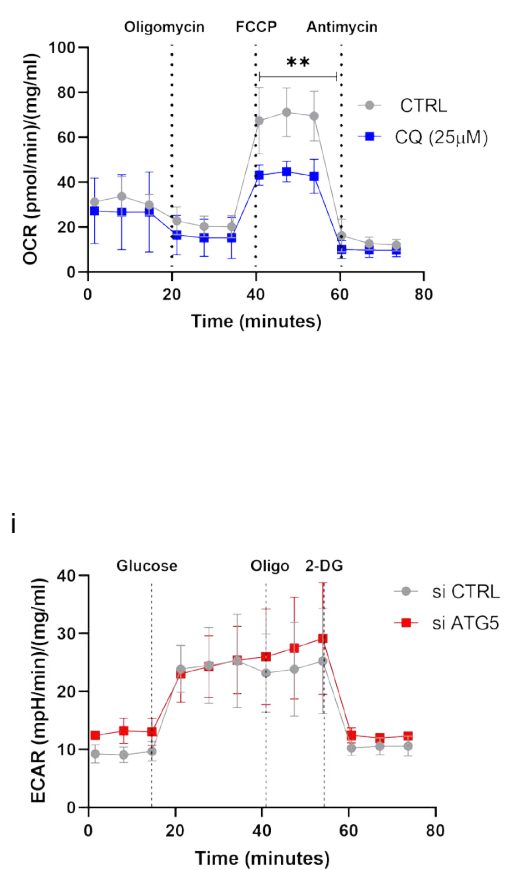

m

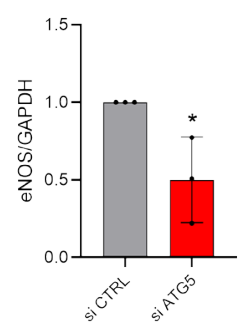

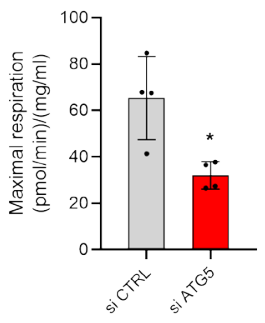

f
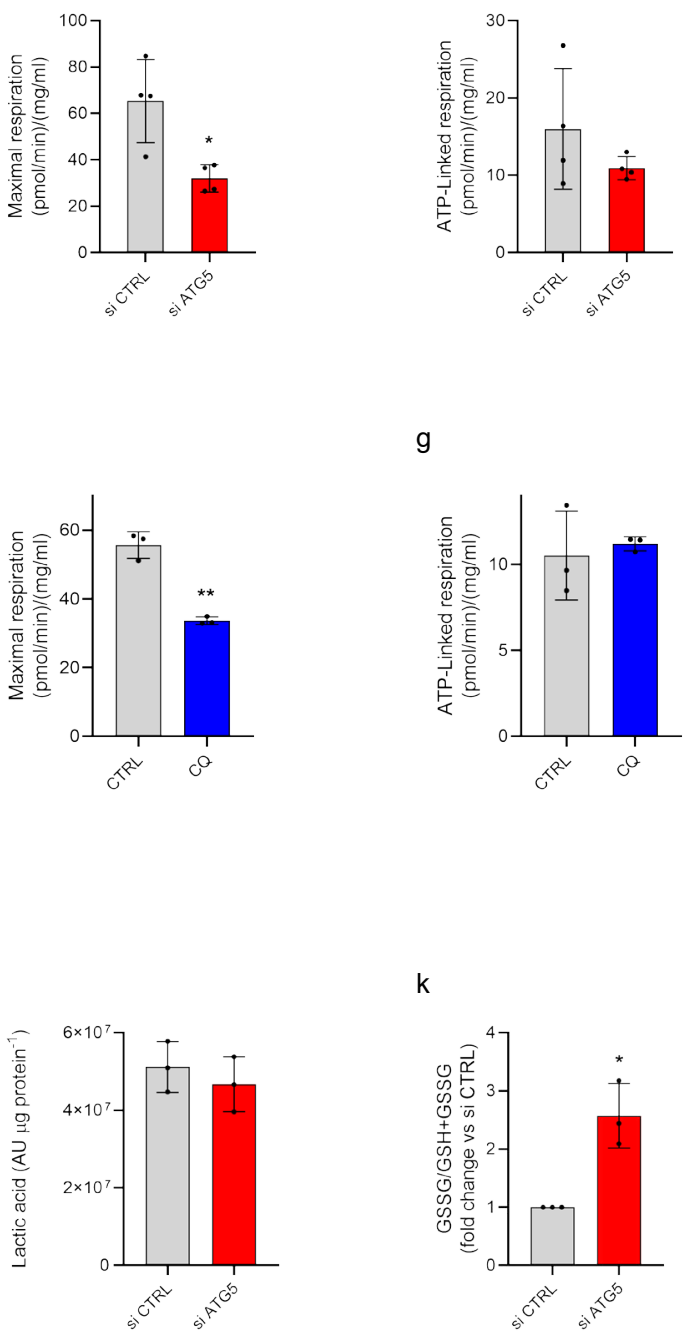

g

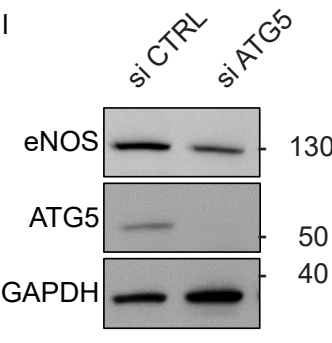

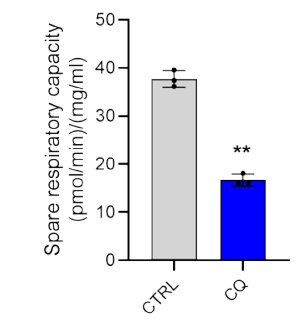

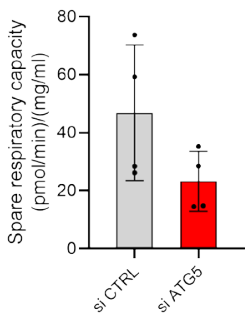

h

30

50

40

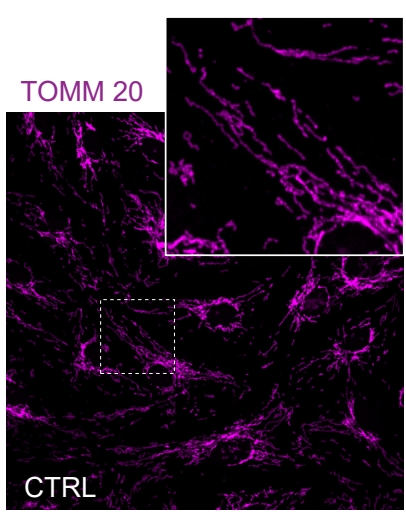




\section{Extended Data Fig.4 | Assessment of mitochondrial respiration parameters in LEC upon ATG5}

inhibition or CQ treatment and etomoxir effects on mitochondrial morphology.

a) OCR of si CTRL or si ATG5 LEC (48h post transfection) determined at baseline, after oligomycin, phenylhydrazone (FCCP) and antimycin treatment, analyzed via the Seahorse XFp Extracellular flux analyzer. Mean $\pm S E M, N=4$ analyzed using unpaired Student's $t$ test, ${ }^{*} p<0.05$. b) Maximal respiration, c) ATP-linked respiration and d) Spare respiratory capacity calculated from OCR in A. Mean $\pm S E M, N=4$ analyzed using unpaired Student's $t$ test, ${ }^{*} \mathrm{p}<0.05$. e) OCR of CTRL or CQ-treated LEC (48h) determined at baseline, after oligomycin, phenylhydrazone (FCCP) and antimycin treatment, analyzed via the Seahorse XFp Extracellular flux analyzer. Mean $\pm \mathrm{SEM}, \mathrm{N}=3$ analyzed using unpaired Student's $t$ test, $* * p<0.01$. f) Maximal respiration, g) ATP-linked respiration and $\mathbf{h}$ ) Spare respiratory capacity calculated from OCR in E. Mean $\pm \mathrm{SEM}, \mathrm{N}=3$ analyzed using unpaired Student's $t$ test, $* * p<0.01$. i) Extracellular acidification rate (ECAR) of si CTRL or si ATG5 LEC determined at baseline, after glucose, oligomycin (Oligo) and 2-deoxy-D-glucose (2-DG) treatment, analyzed via the Seahorse XFp Extracellular flux analyzer. j) Intracellular lactic acid levels and k) Oxidized glutathione calculated by the ratio of oxidized to total glutathione levels. Mean \pm SEM, N=3 analyzed using one sample Student's $t$ test, ${ }^{*} p<0.05$. I-m) Representative blot and quantification of the indicated proteins of si CTRL and si ATG5 LEC. Mean \pm SEM, N=3 analyzed one sample Student's $t$ test ${ }^{*} p<0.05$. $\left.\mathbf{n}-\mathbf{o}\right)$ Representative immunofluorescent images $(n)$ of the mitochondrial protein TOMM20 from untreated (CTRL) LEC or after treatment (48h) with etomoxir (Eto, $100 \mu \mathrm{M})$ and (o) quantification of mitochondrial fragmentation index. Mean $\pm \mathrm{SEM}, \mathrm{N}=3$ analyzed using unpaired Student's $t$ test, ${ }^{*} \mathrm{p}<0.05$. 


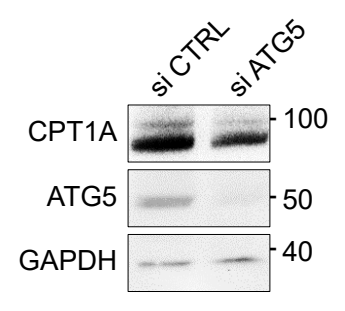

e

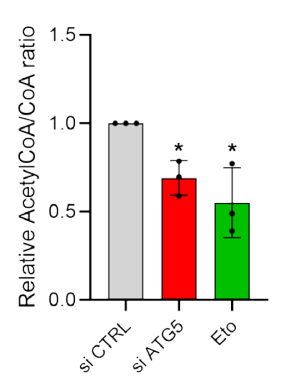

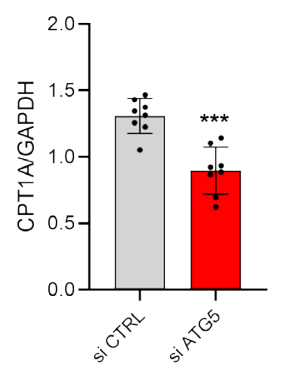

f

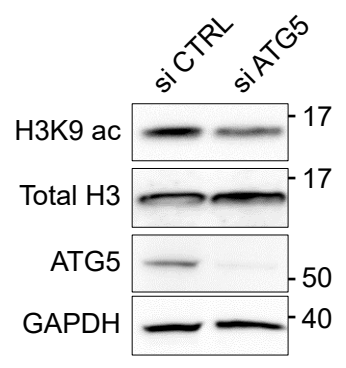

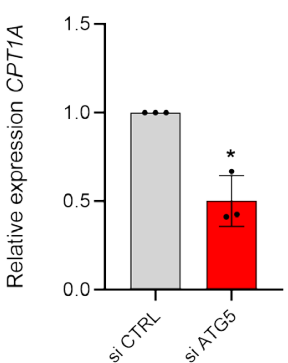

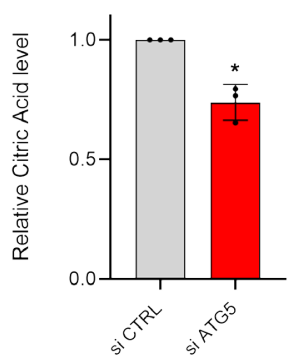

g

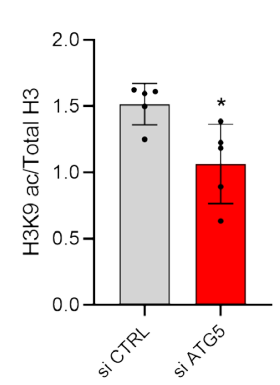

h

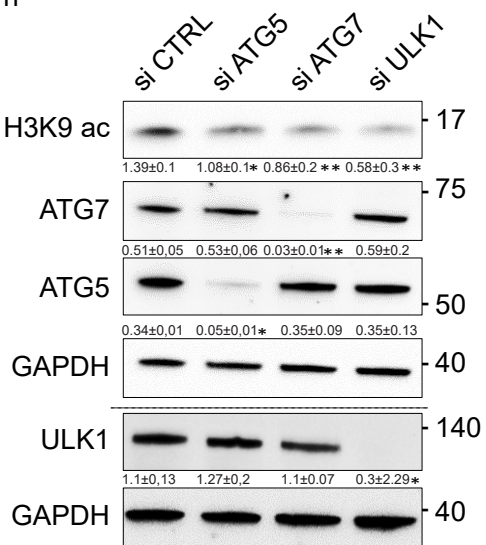

Fig. 5 | Autophagy regulates CPT1A expression and acetyl-CoA levels.

a) Representative immunoblots and $\mathbf{b})$ densitometric analysis of CPT1A levels relative to GAPDH of si CTRL or si ATG5 LEC. Mean \pm SEM, N=3 analyzed using unpaired Student's $t$ test, ${ }^{* * *} p<0.001$. c) mRNA expression of CPT1A (relative to HPRT) in si CTRL or si ATG5 LEC (48h after transfection). Mean \pm SEM, $\mathrm{N}=3$ analyzed using one sample Student's $t$ test, ${ }^{*} p<0.05$. d) Relative Citric acid levels in si CTRL and si ATG5 LEC measured via mass spectrometry. Mean \pm SEM, $N=3$ analyzed using one sample Student's $t$ test, ${ }^{*} \mathrm{p}<0.05$. e) Relative acetyl CoA/CoA ratio measured via mass spectrometry in si CTRL, si ATG5 or etomoxir (Eto $100 \mu \mathrm{M}, 48 \mathrm{~h}$ ) treated LEC. Mean $\pm \mathrm{SEM}, \mathrm{N}=3$ analyzed using one sample Student's $t$ test, ${ }^{*} p<0.05$ vs si CTRL. f) Representative immunoblots and g) Densitometric analysis for H3K9 ac levels relative to total $\mathrm{H} 3$ of si CTRL and si ATG5 LEC (48h after transfection). Mean $\pm \mathrm{SEM}, \mathrm{N}=5$ analyzed using unpaired Student's $t$ test, ${ }^{*} p<0.05$. h) Representative immunoblots for indicated proteins with GAPDH as loading control of si CTRL, si ATG5, si ATG7 or si ULK1 LEC (48h after transfection). Densitometric quantification indicated beneath the blots. Mean $\pm \mathrm{SEM}, \mathrm{N}=6$ analyzed using one way ANOVA with Tukey's test for multiple comparisons, ${ }^{*} p<0.05,{ }^{* *} p<0.01$ vs si CTRL. 

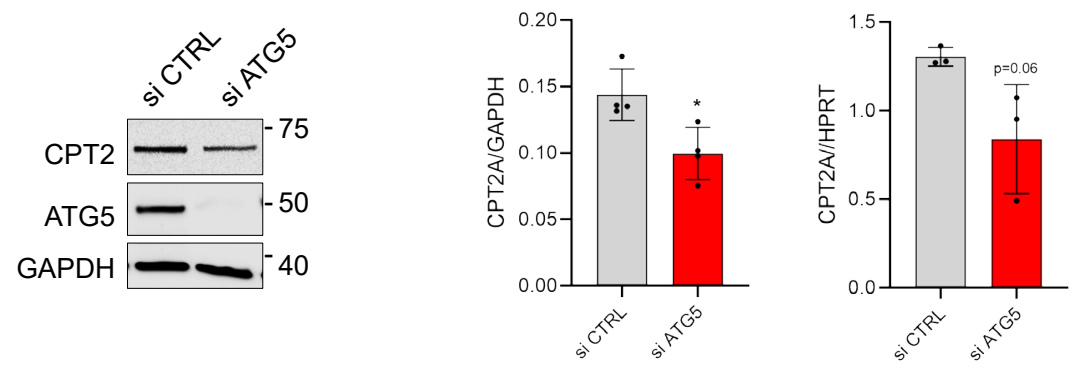

Extended Data Fig.5 | ATG5 silencing attenuates CPT2 expression in LEC.

a) Representative immunoblots and b) densitometric analysis for CPT2 levels of si CTRL or si ATG5 LEC (48h after transfection). Mean $\pm \mathrm{SEM}, \mathrm{N}=3$ analyzed using unpaired Student's $t$ test, ${ }^{*} p<0.05 . \mathrm{c}$ ) mRNA expression of $C P T 2$ (relative to HPRT). Mean $\pm \mathrm{SEM}, \mathrm{N}=3$ analyzed by unpaired Student's $t$ test. 


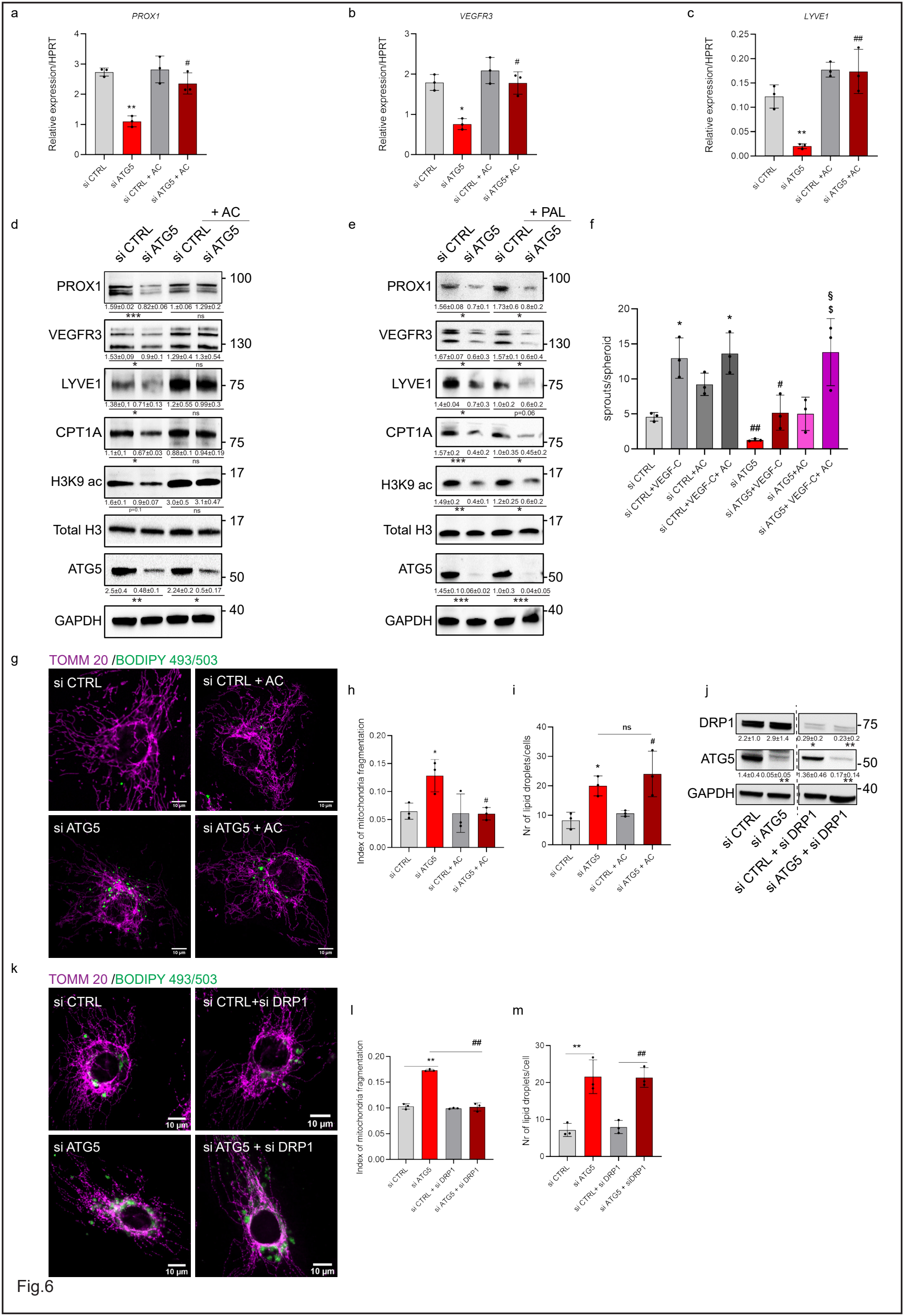


Fig. 6 | Acetate rescues transcriptional and functional defects caused by genetic deficiency of ATG5 in vitro.

(a-d) si CTRL or si ATG5 LEC treated with $20 \mathrm{mM}$ sodium acetate (+ AC) or vehicle (48h after treatment and transfection). a-c) mRNA levels of Prox1, VEGFR3 and LYVE1 (relative to HPRT). Mean $\pm \mathrm{SEM}, \mathrm{N}=3$ analyzed by one-way ANOVA, with Tukey's test for multiple comparisons, *p<0.05 vs si CTRL, **p<0.01 vs si CTRL, $" p<0.05$ vs si ATG5 and ${ }^{\# \#} p<0.01$ vs si ATG5. d) Representative blots for the indicated proteins of si CTRL or si ATG5 LEC treated with $20 \mathrm{mM}$ sodium acetate $(+\mathrm{AC})$ or vehicle $(48 \mathrm{~h}$ after treatment and transfection). Densitometric quantifications indicated beneath the blots. Mean \pm SEM, $\mathrm{N} \geq 3$ analyzed by one-way ANOVA, with Tukey's test for multiple comparisons, ${ }^{*} p<0.05, * * * p<0.001$ vs si CTRL. e) Representative blots of si CTRL and si ATG5 LEC supplemented with 500nM palmitate (+ $\mathrm{PAL}$ ) or BSA (48h after treatment and transfection). Densitometric quantifications are indicated beneath the blots. Mean $\pm \mathrm{SEM}, \mathrm{N} \geq 3$ analyzed by one-way ANOVA, with Tukey's test for multiple comparisons. ${ }^{*} p<0.05,{ }^{* *} p<0.01$ vs corresponding control. f) Quantification of sprouts per spheroid (from Figure S6N). Mean \pm SEM, N=3 analyzed using Two-way ANOVA, followed by the Holm-Sidak test for post hoc multiple comparisons ${ }^{*} p<0.05$ vs si CTRL unstimulated, ${ }^{\#} p<0.05$ and ${ }^{\# \#} p<0.01$ vs si CTRL + VEGF-C , $\$ p<0.05$ vs si ATG5 + VEGF-C and ${ }^{\S} \mathrm{p}<0.05$ vs si ATG5 + AC. g) Representative double immunofluorescent images for the mitochondrial protein TOMM20 and BODIPY 493/503 from si CTRL and si ATG5 LEC under basal conditions and after sodium acetate (+ AC) supplementation (48h after treatment and transfection). Scale bars represent $10 \mu \mathrm{m}$. h) Quantification of mitochondrial fragmentation index. Mean $\pm \mathrm{SEM}, \mathrm{N}=3$ analyzed by one-way ANOVA, with Tukey's test for multiple comparisons, ${ }^{*} p<0.05$ vs si CTRL, ${ }^{\#} p<0.05$ vs si ATG5. i) Quantification of lipid droplet number per cell. Mean $\pm \mathrm{SEM}, \mathrm{N}=4$ analyzed by one-way ANOVA, with Tukey's test for multiple comparisons, ${ }^{*} \mathrm{p}<$ 0.05 vs si CTRL, ${ }^{\# \#}$ p < 0.01 vs si CTRL+AC. j) Representative blots of si CTRL, si ATG5, si CTRL + si DRP1 and si ATG5 +si DRP1 $48 \mathrm{~h}$ post transfection. Densitometric quantifications are indicated beneath the blots. Mean $\pm S E M, N \geq 3$ analyzed by one-way ANOVA, with Tukey's test for multiple comparisons. ${ }^{*} p$ $<0.05$ vs si CTRL, ${ }^{* *} p<0.01$ vs si ATG5, ${ }^{* *} p<0.01$ vs si CTRL, ${ }^{* *} p<0.01$ vs si CTRL+si DRP1. k) Representative immunofluorescent images of double stainings for the mitochondrial protein TOMM20 and BODIPY 493/503 from si CTRL and si ATG5, si CTRL+si DRP1 and si ATG5+ si DRP1 LEC (48h after transfection). I) Quantification for mitochondrial index of fragmentation. Mean $\pm S E M, N=3$ analyzed by one-way ANOVA, with Tukey's test for multiple comparisons, ${ }^{* *} p<0.01$ vs si CTRL, ${ }^{\# \#} p<0.01$ vs si ATG5. $\mathrm{m}$ ) Quantification of lipid droplet number per cell. Mean $\pm \mathrm{SEM}, \mathrm{N}=3$ analyzed by one-way ANOVA, with Tukey's test for multiple comparisons, ${ }^{* *} p<0.01$ vs si CTRL, ${ }^{\# \#} p<0.01$ vs si CTRL + si DRP1. 


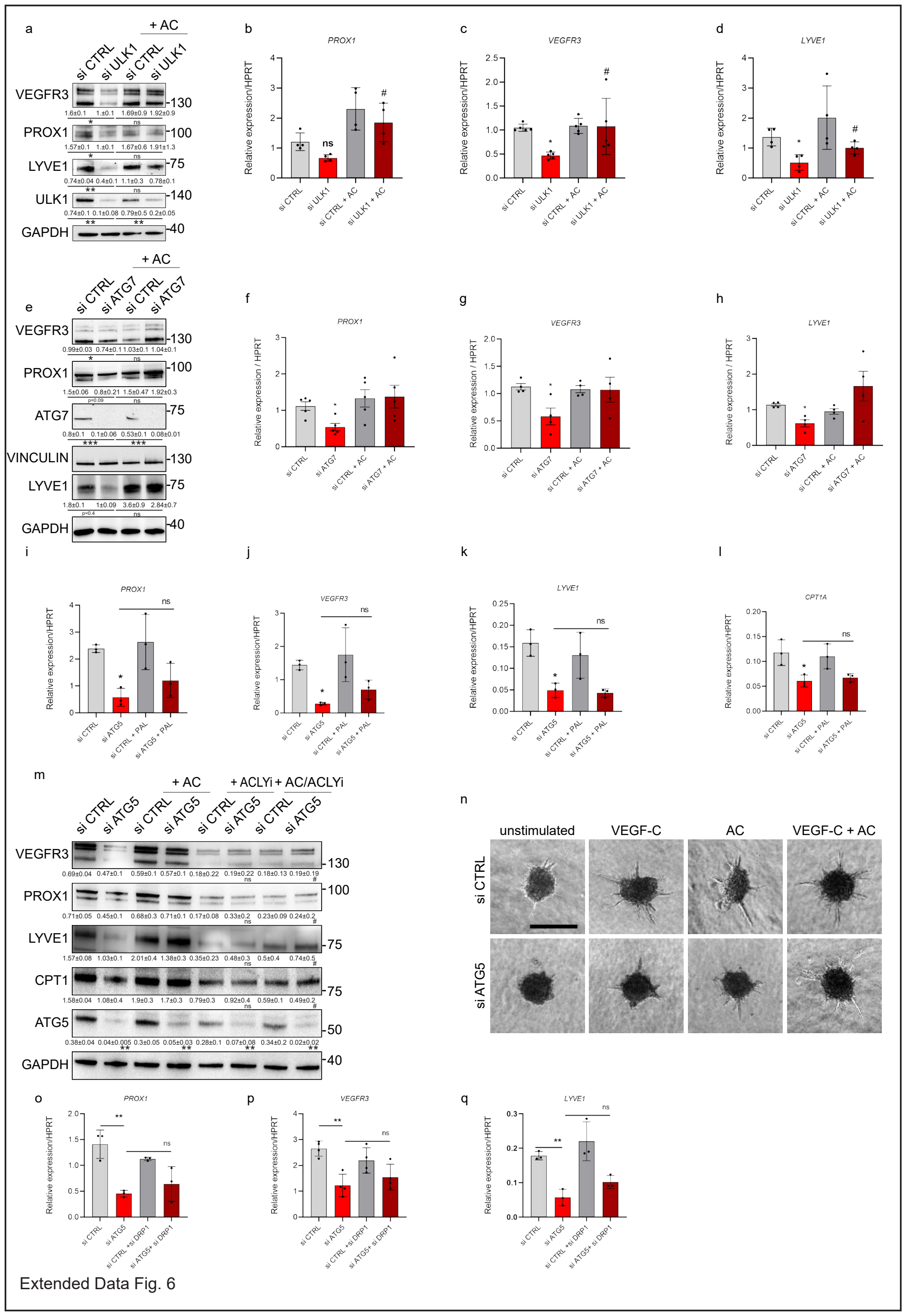


Extended Data Fig.6 | Rescue of PROX-1 driven lymphatic markers by acetate in autophagy compromised LEC.

a-h) si CTRL, si ULK1 or si ATG7 LEC treated with $20 \mathrm{mM}$ sodium acetate (AC) or vehicle for 48h. $(\mathrm{a}, \mathrm{e})$ Representative immunoblots for indicated proteins of si CTRL, si ULK1 or si ATG7 LEC treated with $20 \mathrm{mM} \mathrm{AC}(+\mathrm{AC})$ or vehicle (48h after treatment and transfection) with GAPDH as loading control. Densitometric quantifications indicated beneath the blots. Mean $\pm \mathrm{SEM}, \mathrm{N}=3$ analyzed by one-way ANOVA, with Tukey's test for multiple comparisons, ${ }^{*} p<0.05$ vs si CTRL, ${ }^{*} p<0.01$ vs si CTRL. (b-d) and (f-h) RT-qPCR analysis for PROX1, VEGFR3 and LYVE1 genes (relative to HPRT). Mean $\pm \mathrm{SEM}, \mathrm{N} \geq 4$ analyzed by one-way ANOVA, with Tukey's test for multiple comparisons, * $p<0.05$ vs si CTRL and ${ }^{\#} p<$ 0.05 vs si ULK1. (i-I) RT-pPCR analysis of si CTRL and si ATG5 LEC supplemented with 500 nM palmitate (+ PAL) or BSA (48h after treatment and transfection) for PROX1, VEGFR3 and LYVE1 and CPT1A genes (relative to $H P R T$ ). Mean $\pm \mathrm{SEM}, \mathrm{N} \geq 4$ analyzed by one-way ANOVA, with Tukey's test for multiple comparisons, $* p<0.05$ vs si CTRL. $m$ ) Representative immunoblot of si CTRL and si ATG5 upon ACLY inhibitor, $A C$ alone or in combination (10 $\mu \mathrm{M}$ and $20 \mathrm{mM}$ respectively, 48h). Densitometric quantifications indicated beneath the blots. Mean $\pm \mathrm{SEM}, \mathrm{N} \geq 3$ analyzed by two-way ANOVA, with Tukey's test for multiple comparisons, ${ }^{* *} p<0.01$ vs si CTRL and ${ }^{*} p<0.05$ vs si ATG5 + AC and ns vs si ATG5. n) Representative spheroid images from si CTRL and si ATG5 LEC under basal conditions, stimulated with recombinant VEGF-C $(100 \mathrm{ng} / \mathrm{mL})$ alone, supplemented with $A C$ alone or in combination (48h post supplementation). Scale bar represents $100 \mu \mathrm{m}$. $\mathbf{0}-\mathbf{q}) \mathrm{RT}-\mathrm{qPCR}$ analysis of si CTRL, si ATG5, si CTRL + si DRP1 and si ATG5+ si DRP1 after 48h. mRNA expression of PROX1, VEGFR3 and LYVE1 (relative to HPRT). Mean $\pm \mathrm{SEM}, \mathrm{N} \geq 3$ analyzed by one-way ANOVA, with Tukey's test for multiple comparisons, ${ }^{* *} p<0.01$ vs. si CTRL. 

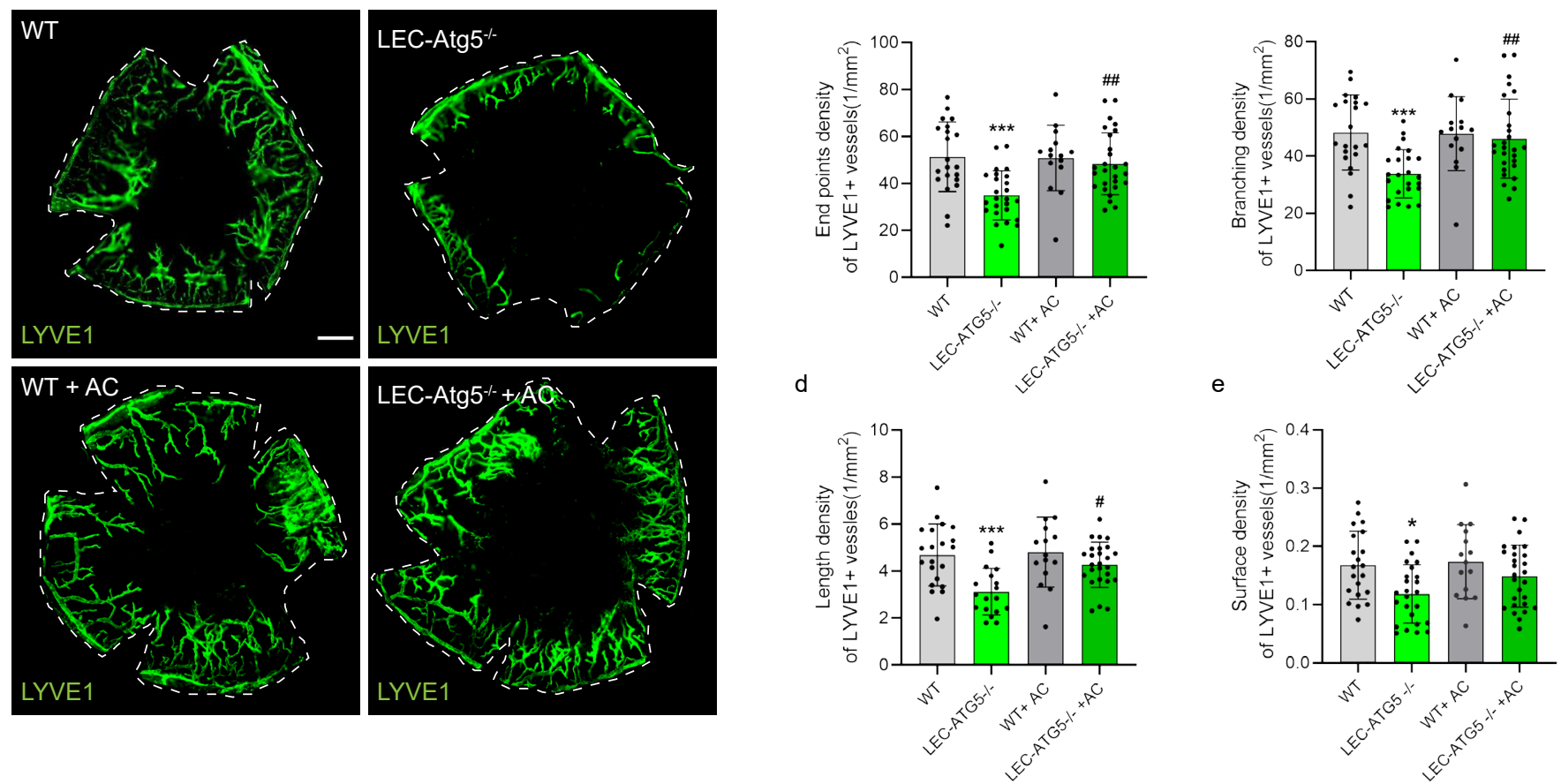

g
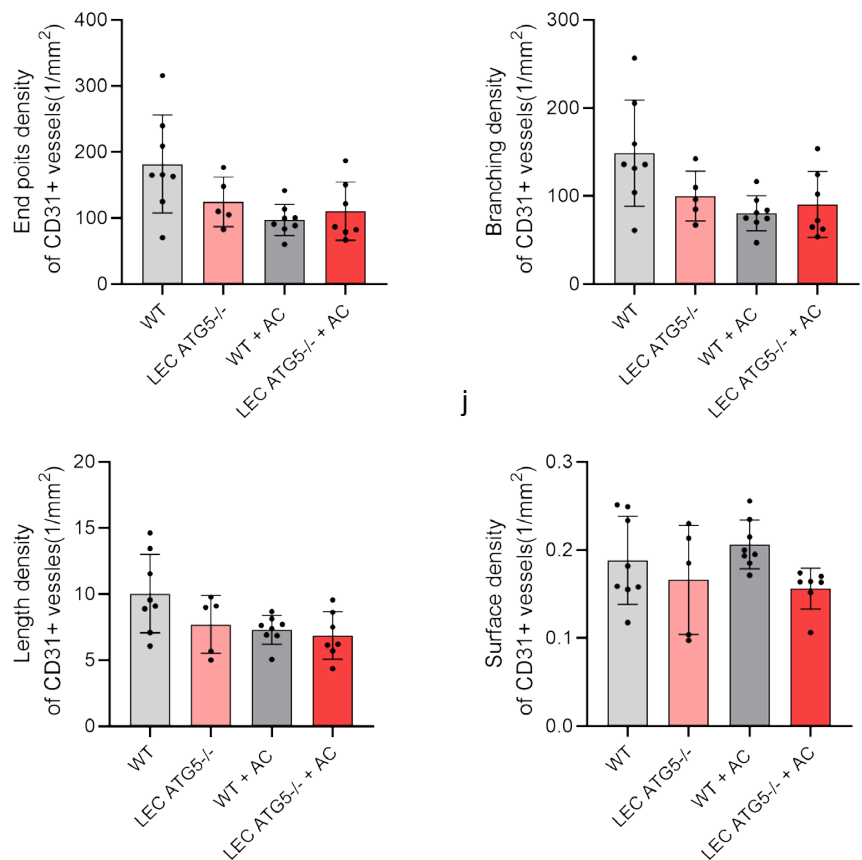

Fig. 7 
Fig. 7 | Acetate rescues corneal lymphangiogenesis in LEC-ATG5 $\%$ mice.

Corneal cauterization-induced injury in WT and LEC-Atg5 ${ }^{-1-}$ mice treated with vehicle or sodium acetate (i.p. AC; $400 \mu$ l of $0.5 \mathrm{M}$ daily) 8 days post corneal cauterization. A) Representative whole corneal images of LYVE1+ lymphatic outgrowth (green) from WT and LEC-Atg5 ${ }^{-1-}$ treated with vehicle or sodium acetate $(A C), 8$ days post corneal cauterization. Scale bar represents $1 \mathrm{~mm}$. (B-E) Quantification of the number of end points, number of branch points, average of cumulative length and surface density for LYVE1+ lymphatic vessels. Each data point on graphs represents one cornea. Mean $\pm \mathrm{SEM}, \mathrm{N}=21$ for WT, N=24 for LEC-Atg5 $\%, N=15$ for WT + AC and N=27 for LEC-Atg5 $\%$ + AC analyzed using one-Way ANOVA corrected for multiple comparisons using Tukey's test * $p<0.05$ vs WT, ***p $<0.001$ vs WT, " $p$ $<0.05$ vs LEC-Atg5 $\%$ and ${ }^{\# \#} \mathrm{p}<0.01$ vs LEC-Atg5 $\%$. F) Representative double immunofluorescent images of corneal sections dissected from WT and LEC-Atg5 $5^{-1}$ treated with vehicle or sodium acetate (AC), stained for LYVE1 (green) and CD31 (red), 8 days post corneal cauterization. Scale bar $100 \mu \mathrm{m}$. G-J) Quantification for the number of end points, number of branch points, average of cumulative length and surface density for the CD31+ blood vessels. Mean $\pm \mathrm{SEM}, \mathrm{N}=8$ for $\mathrm{WT}, \mathrm{N}=5$ for $\mathrm{LEC}-\mathrm{Atg} 5^{-/}, \mathrm{N}=8$ for WT $+A C$ and $\mathrm{N}=7$ for LEC-Atg5 $\%+A C$ analyzed using one-Way ANOVA corrected for multiple comparisons using Tukey's test. 
$$
\text { a }
$$
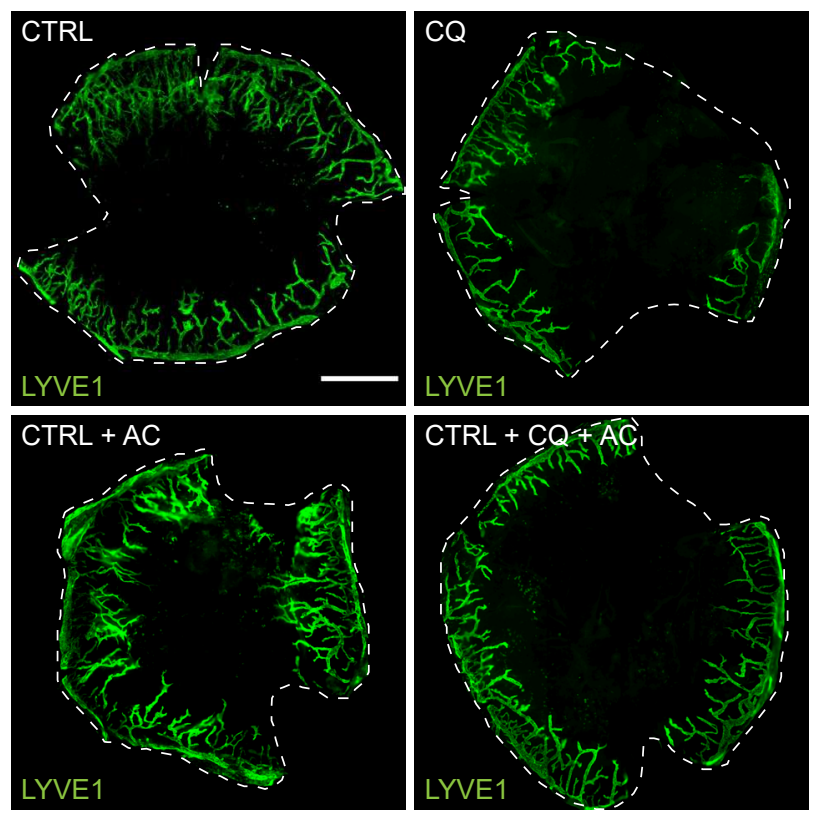

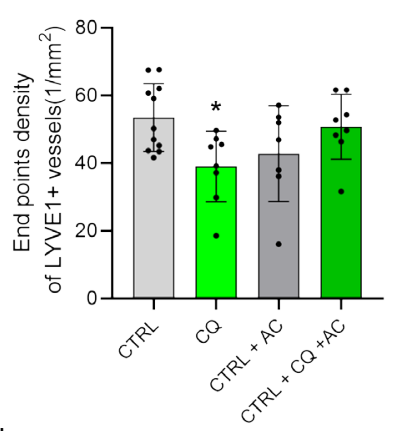

d

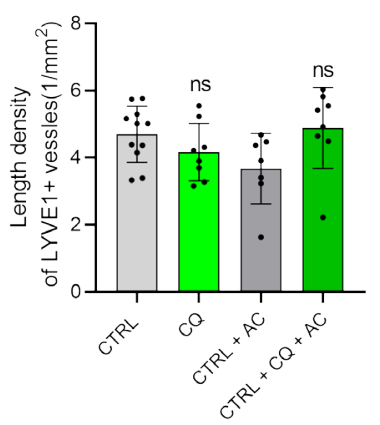

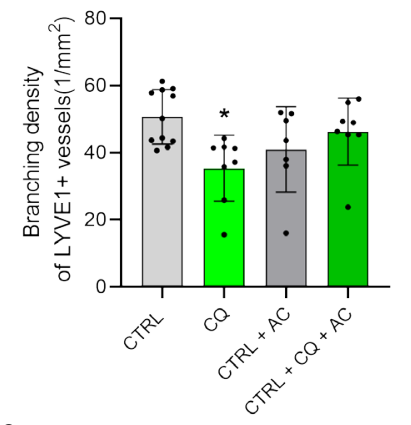

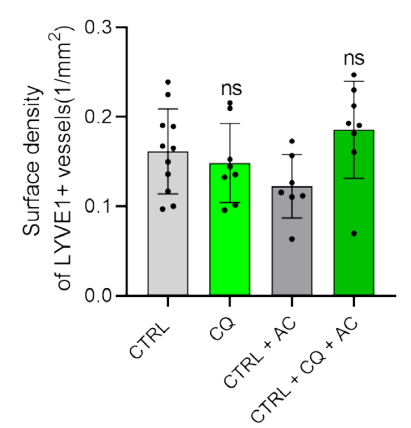

Extended Data Fig.7 | Acetate partly rescues injury-driven lymphangiogenesis in chloroquine treated mice.

a) Representative whole corneal images of LYVE1+ lymphatic outgrowth (green) in vehicle control mice (CTRL) and mice daily treated with chloroquine (CQ, $50 \mathrm{mg} / \mathrm{kg}$ i.p), sodium acetate (AC, $400 \mu \mathrm{l} 0.5$ $\mathrm{M}$ i.p) or in combination $\mathrm{CQ}+\mathrm{AC}$ analyzed 8 days post corneal cauterization. Scale bar represents $1 \mathrm{~mm}$. (b-e) Quantification of the number of end points, number of branch points, average of cumulative length and surface density for LYVE1+ lymphatic vessels. Each data point on graphs represents one cornea. Mean $\pm \mathrm{SEM}, \mathrm{N}=11$ for $\mathrm{CTRL}, \mathrm{N}=8$ for $\mathrm{CQ}, \mathrm{N}=7$ for $\mathrm{CTRL}+\mathrm{AC}$ and $\mathrm{N}=8$ for $\mathrm{CQ}+\mathrm{AC}$ analyzed using one-Way ANOVA corrected for multiple comparisons using Tukey's test, * $p<0.05$ vs CTRL. 

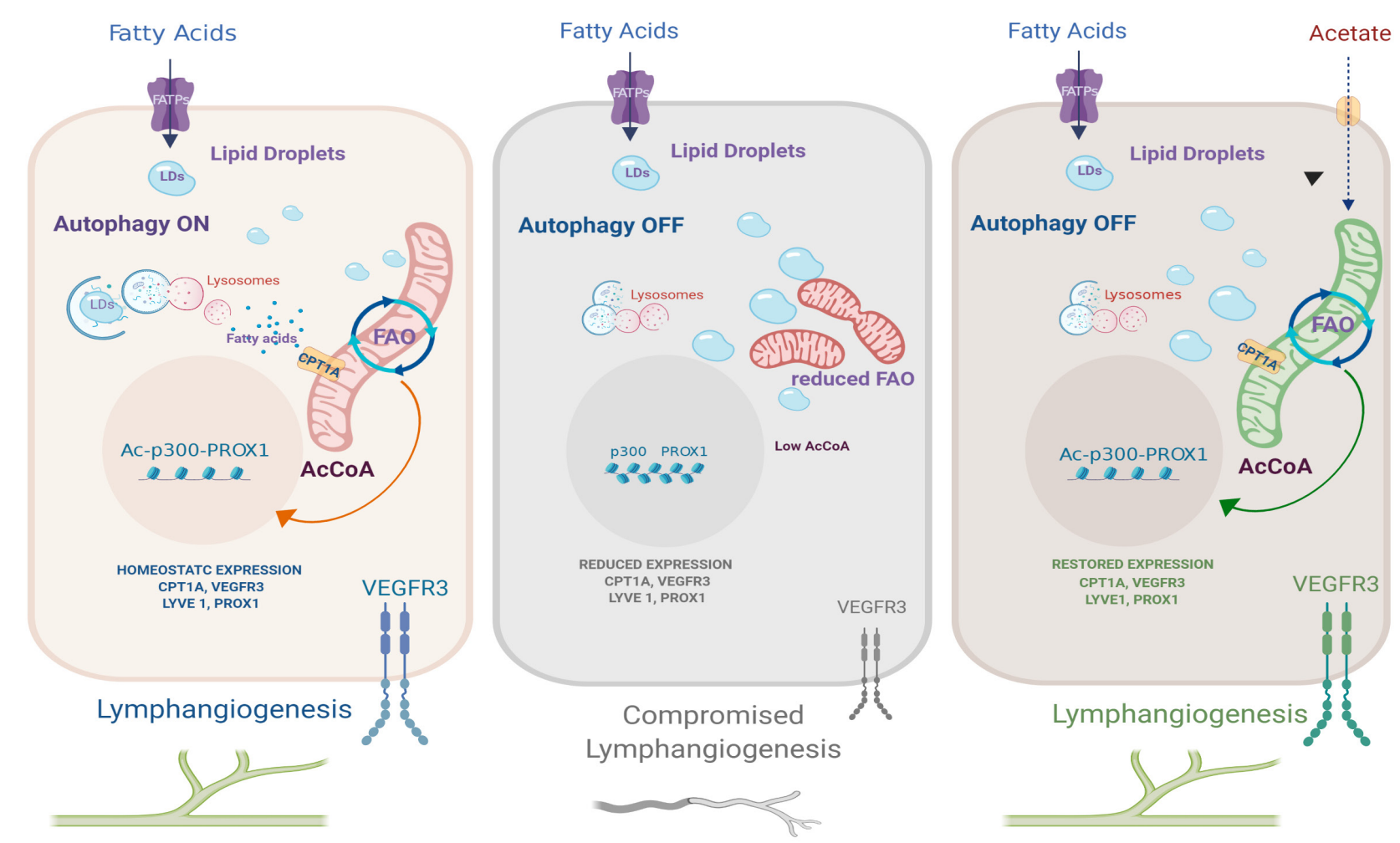

Fig. 8 | Lipophagy maintains lymphatic identity and lymphangiogenesis by supplying fatty acids to the mitochondria for fatty acid oxidation and PROX1-mediated gene expression.

The figure depicts the main phenotypes of (left to right) autophagy-proficient LEC (autophagy ON), autophagy-deficient LEC (autophagy OFF) and their rescue with acetate, described in the study. Under homeostatic conditions (left panel) constitutive level of autophagy favors the turnover of lipid droplets (LDs) in order to supply free fatty acids to the mitochondria and foster fatty acid oxidation (FAO). The actively respiring elongated mitochondria replete the acetyl-CoA pools which is used by the acetyltransferase p300 to acetylate histones at PROX1-target genes. This autophagy regulated mechanism maintains PROX1-driven expression of lymphatic markers and VEGFR3-mediated lymphangiogenic signaling. When autophagy is compromised (center panel) LDs turnover is impaired causing accumulation of LDS in the cytosol. Under these conditions the ability of mitochondria to perform FAO is compromised, the mitochondrial network is fragmented and acetyl-CoA levels drop to levels unable to support transcription of PROX1-driven lymphatic gene networks and lymphangiogenesis. This phenotype can be rescued by feeding LEC with the acetyl-CoA precursor acetate (right panel). Acetate rescues mitochondrila morphology and FAO and recovers PROX1mediated expression of lymphatic genes, thus bypassing the lymphagiogenesis defects caused by genetic loss of autophagy. 
Figures

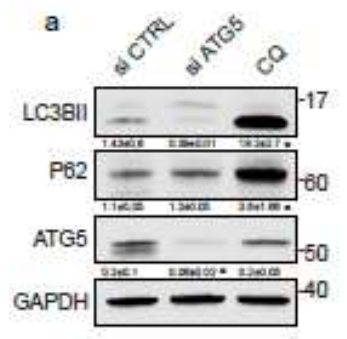

b
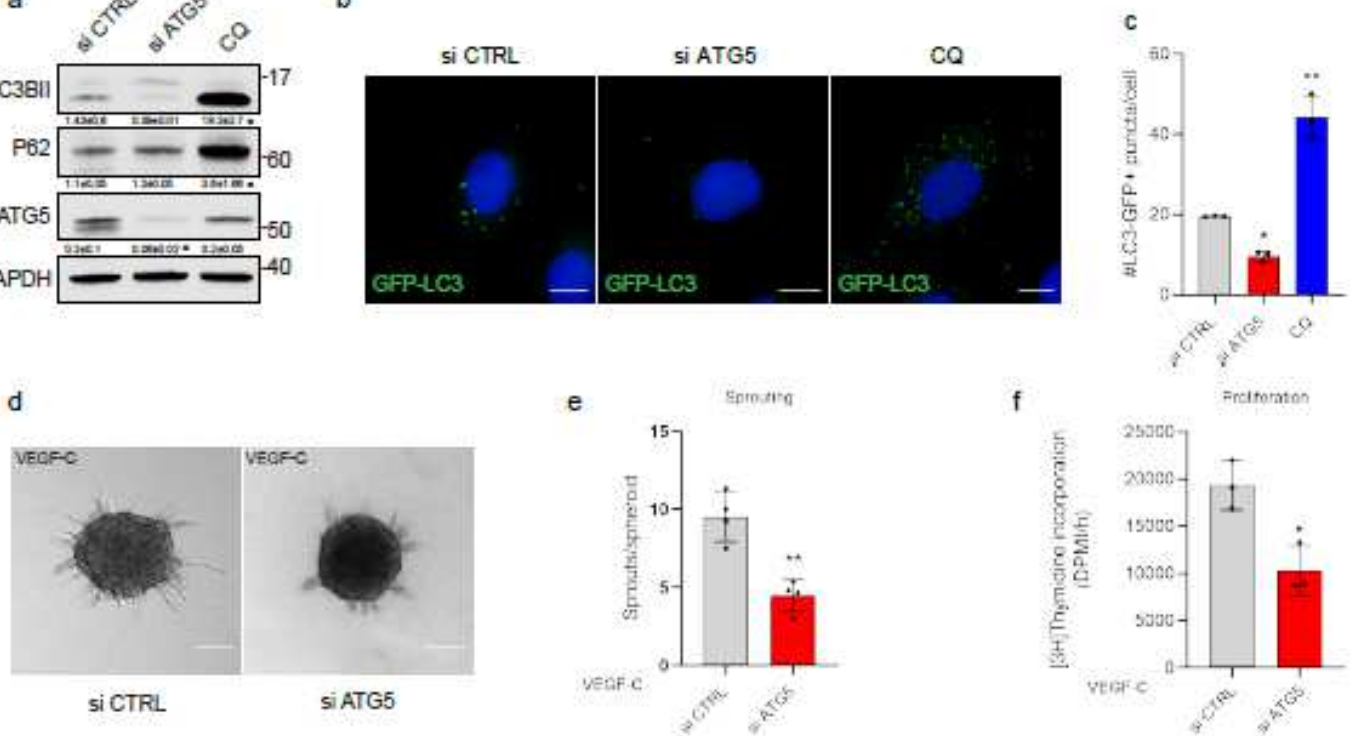

9
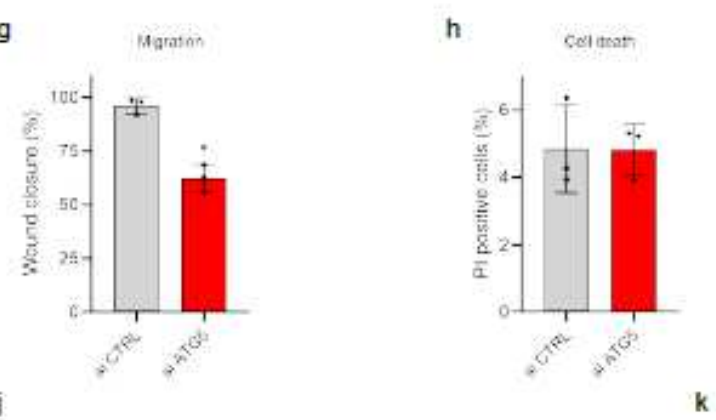

si CTRL SI ATG5
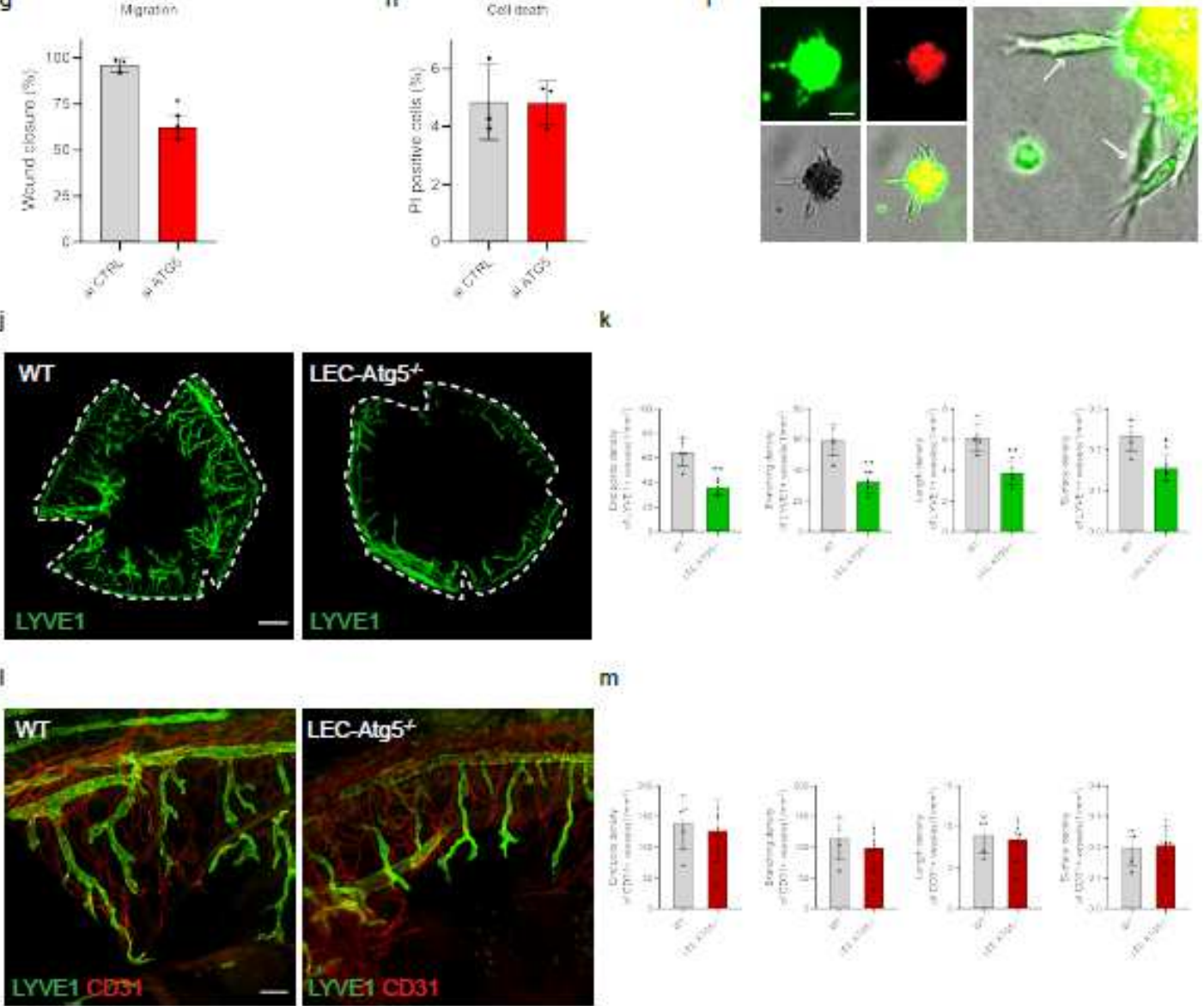

$\mathrm{m}$

Figure 1

Please manuscript .pdf for full figure caption 
a

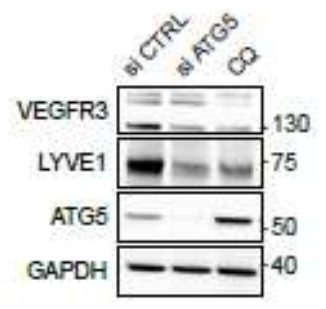

d
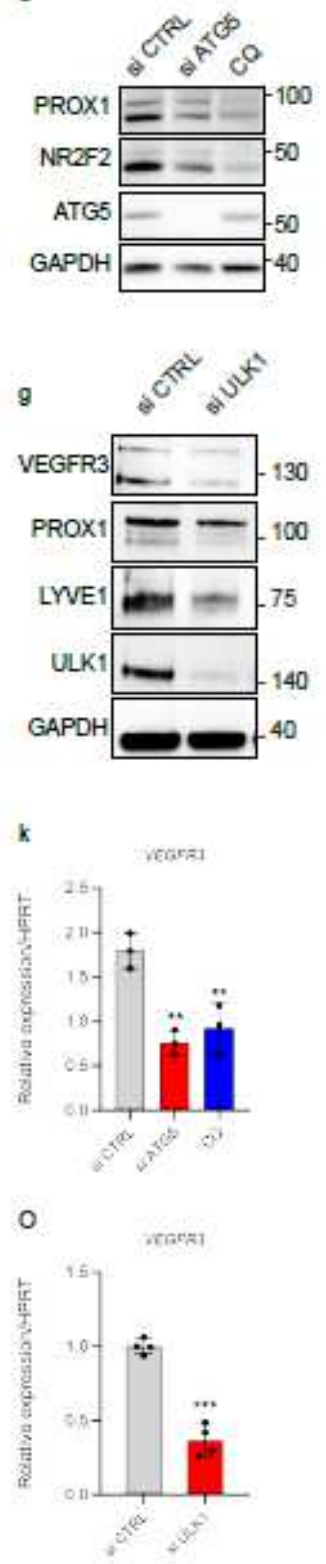
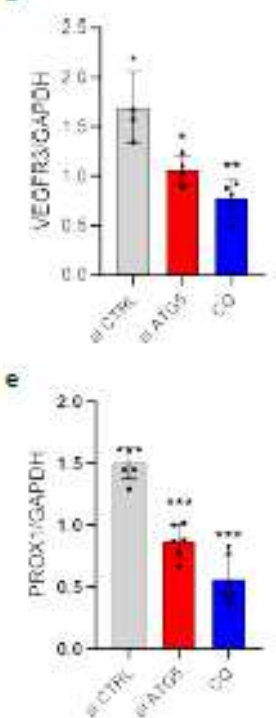
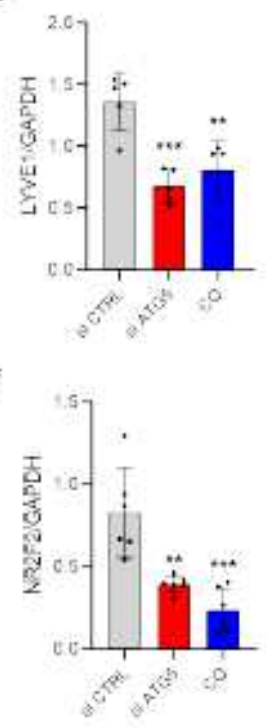

h
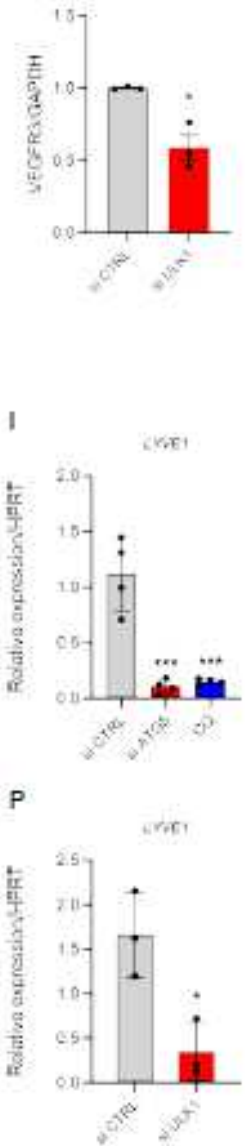
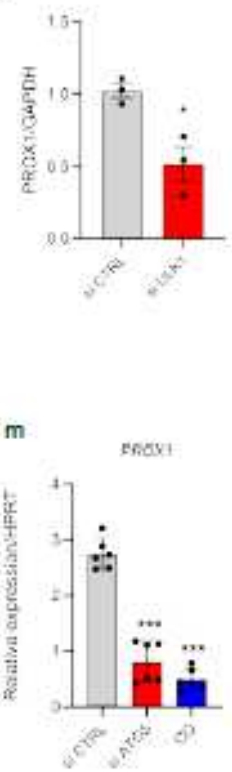

Q

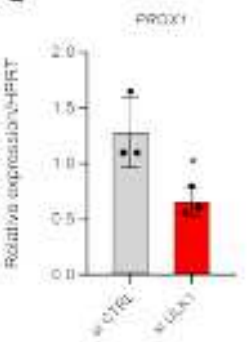

i

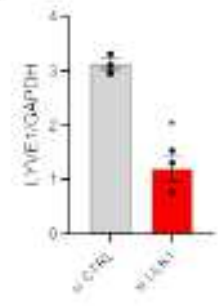

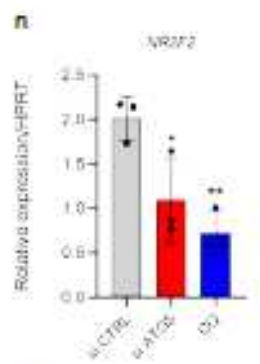

R

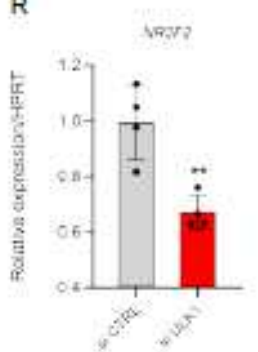

Figure 2

Please manuscript .pdf for full figure caption 

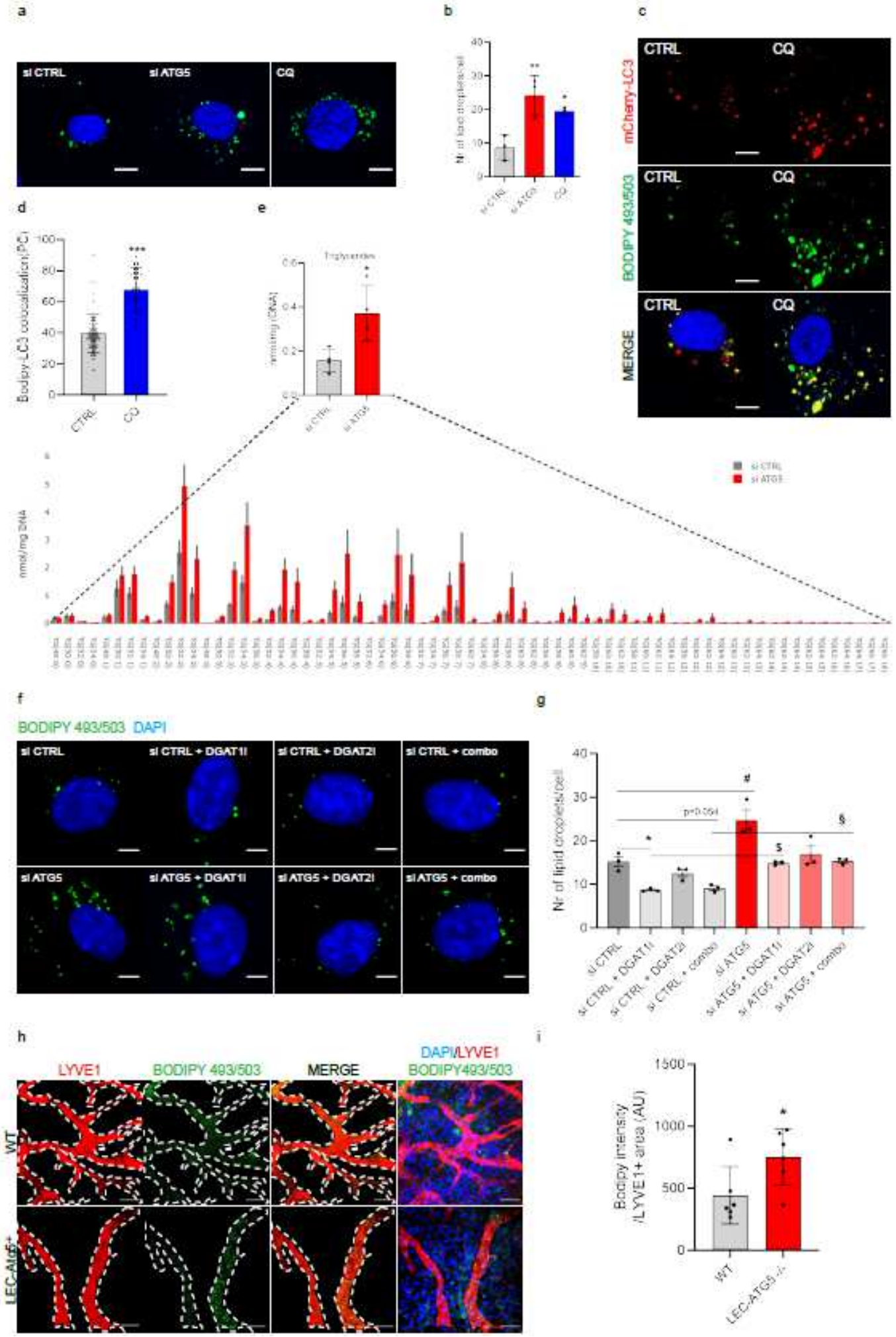

Figure 3

Please manuscript .pdf for full figure caption 

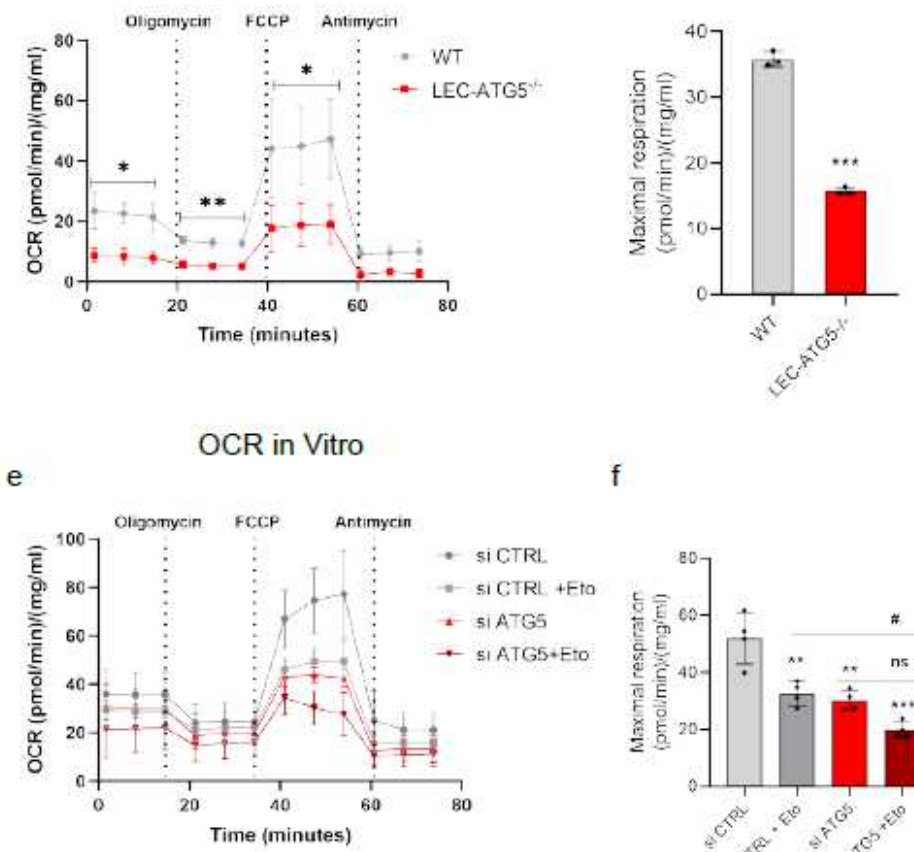

f
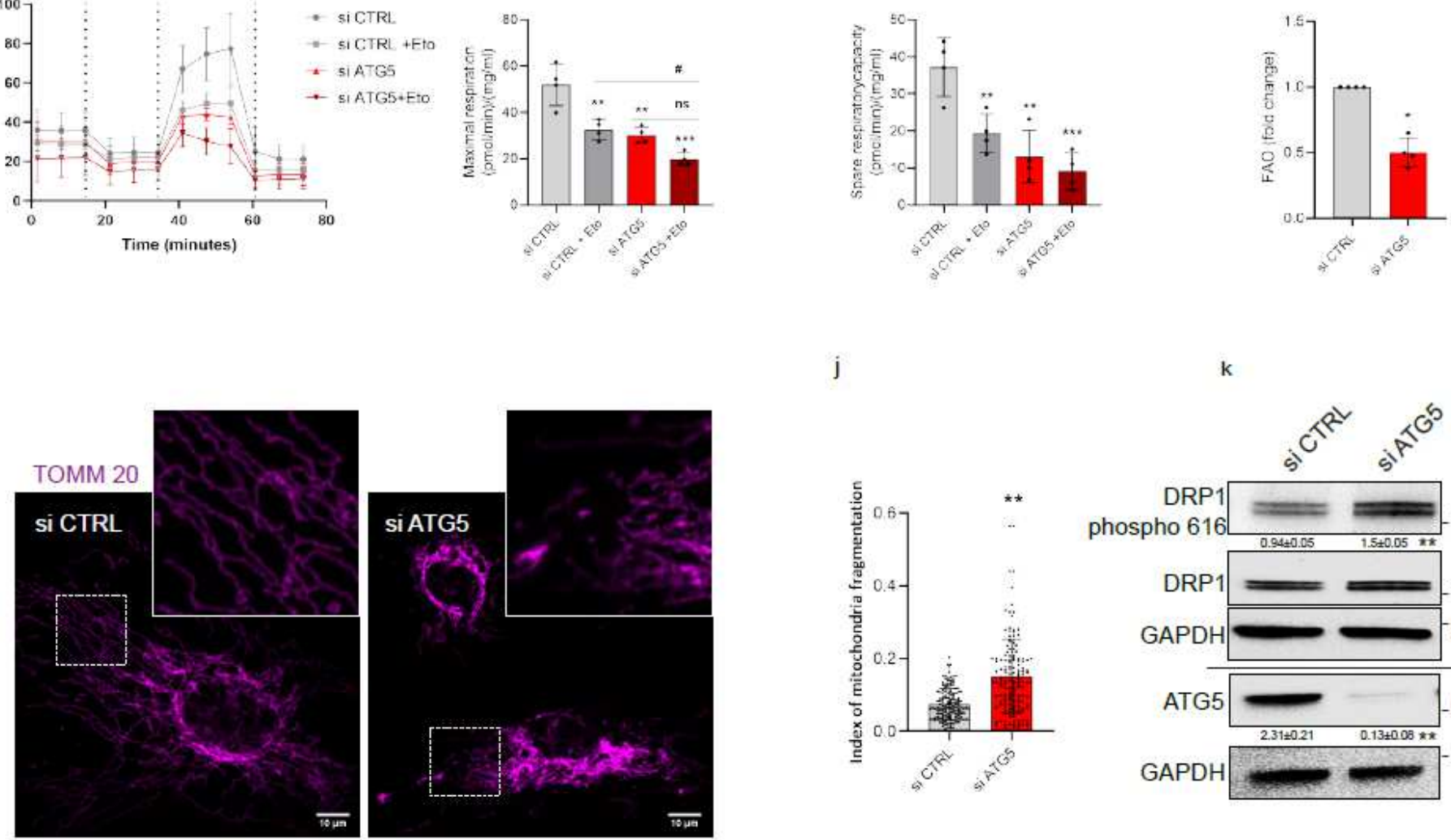

j

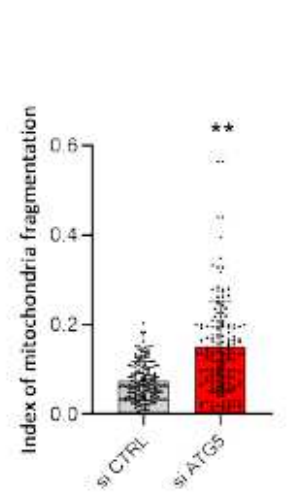

Figure 4

Please manuscript .pdf for full figure caption 

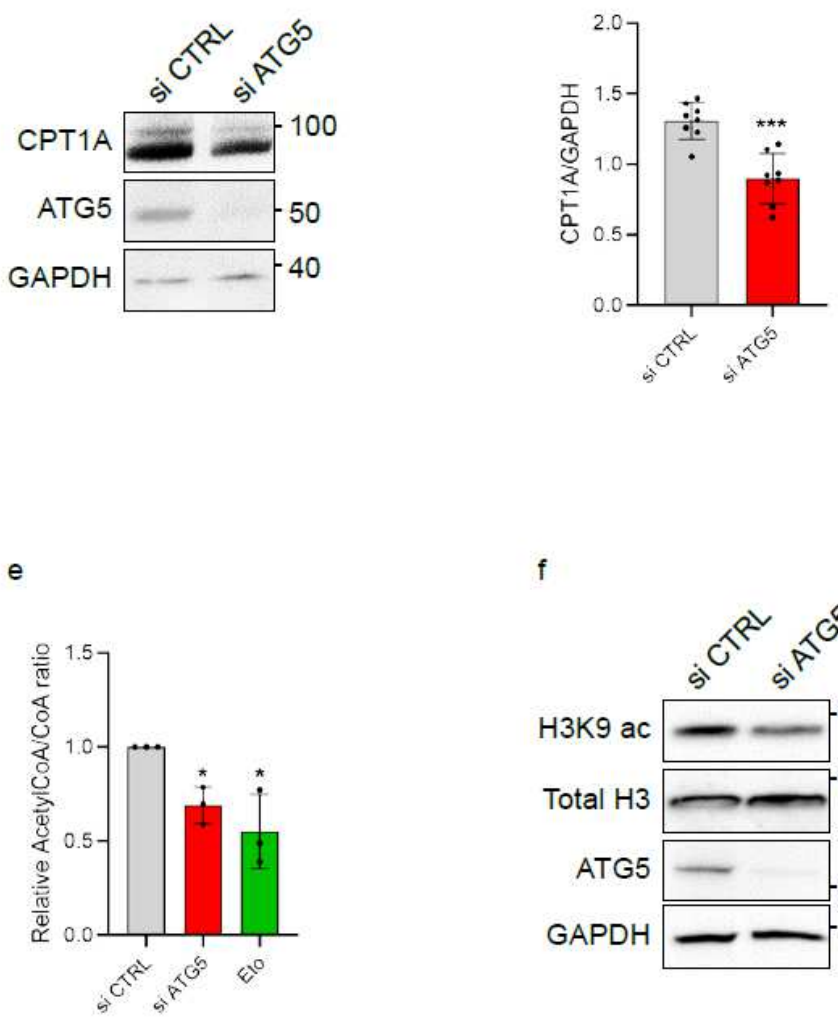

c

g

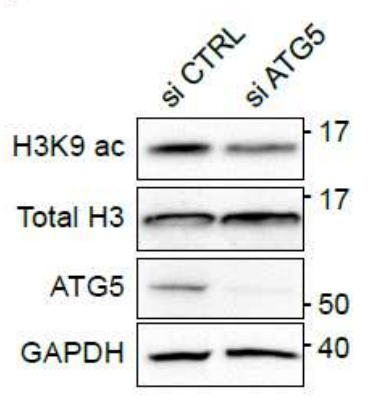

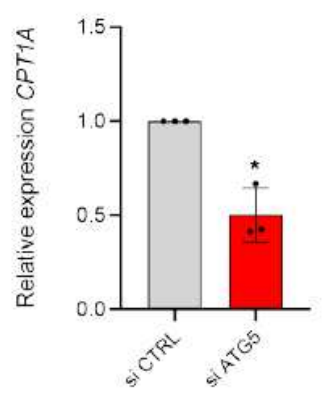

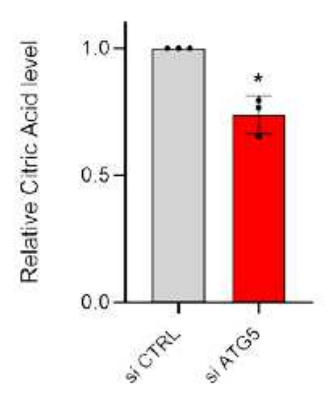

d

h

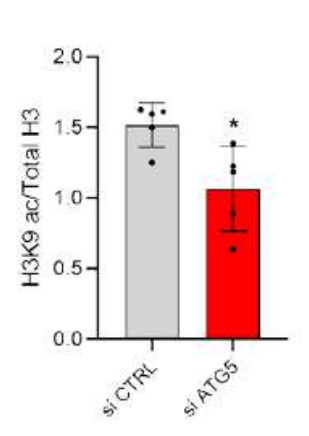

Figure 5

Please manuscript .pdf for full figure caption 


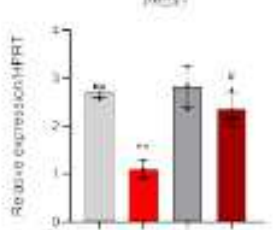

d

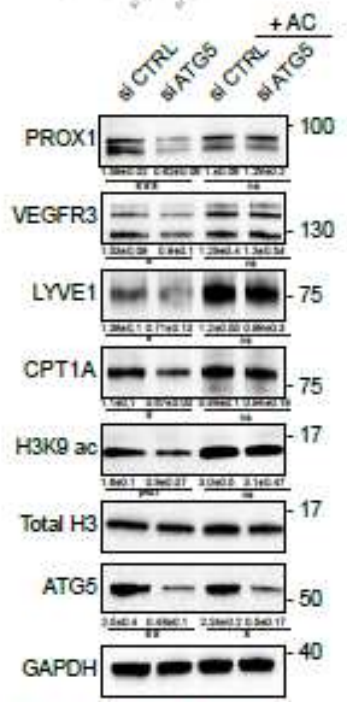

g
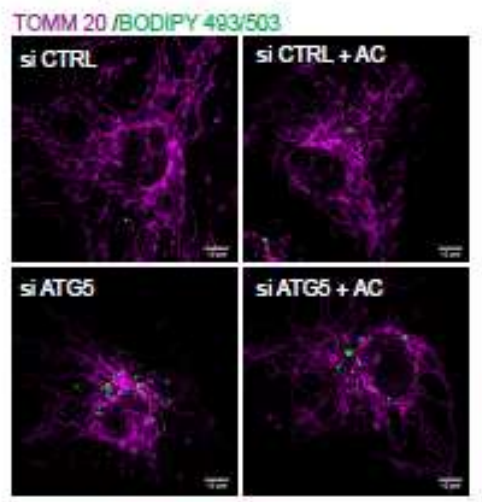

k

TOMM 20 /BOOIPY $493 / 503$

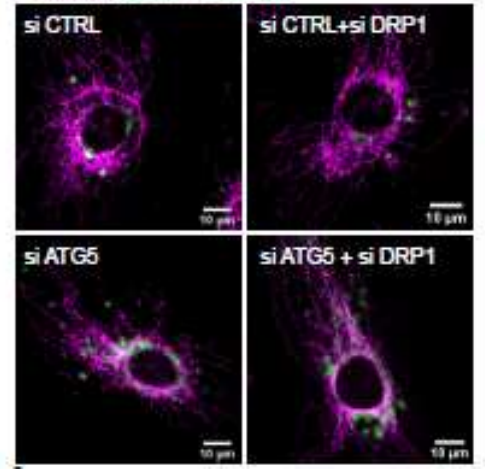

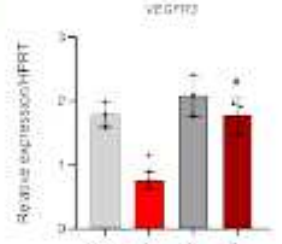

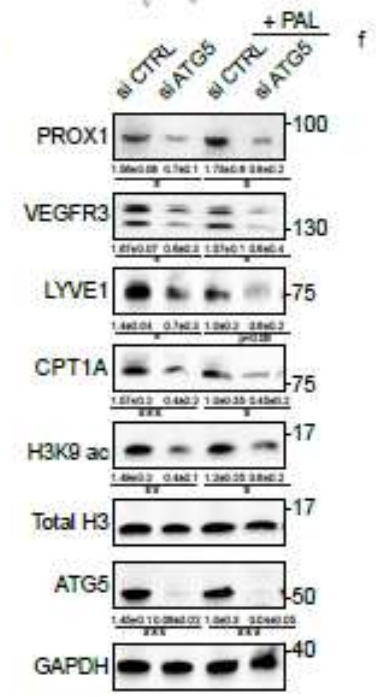

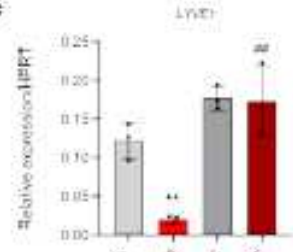
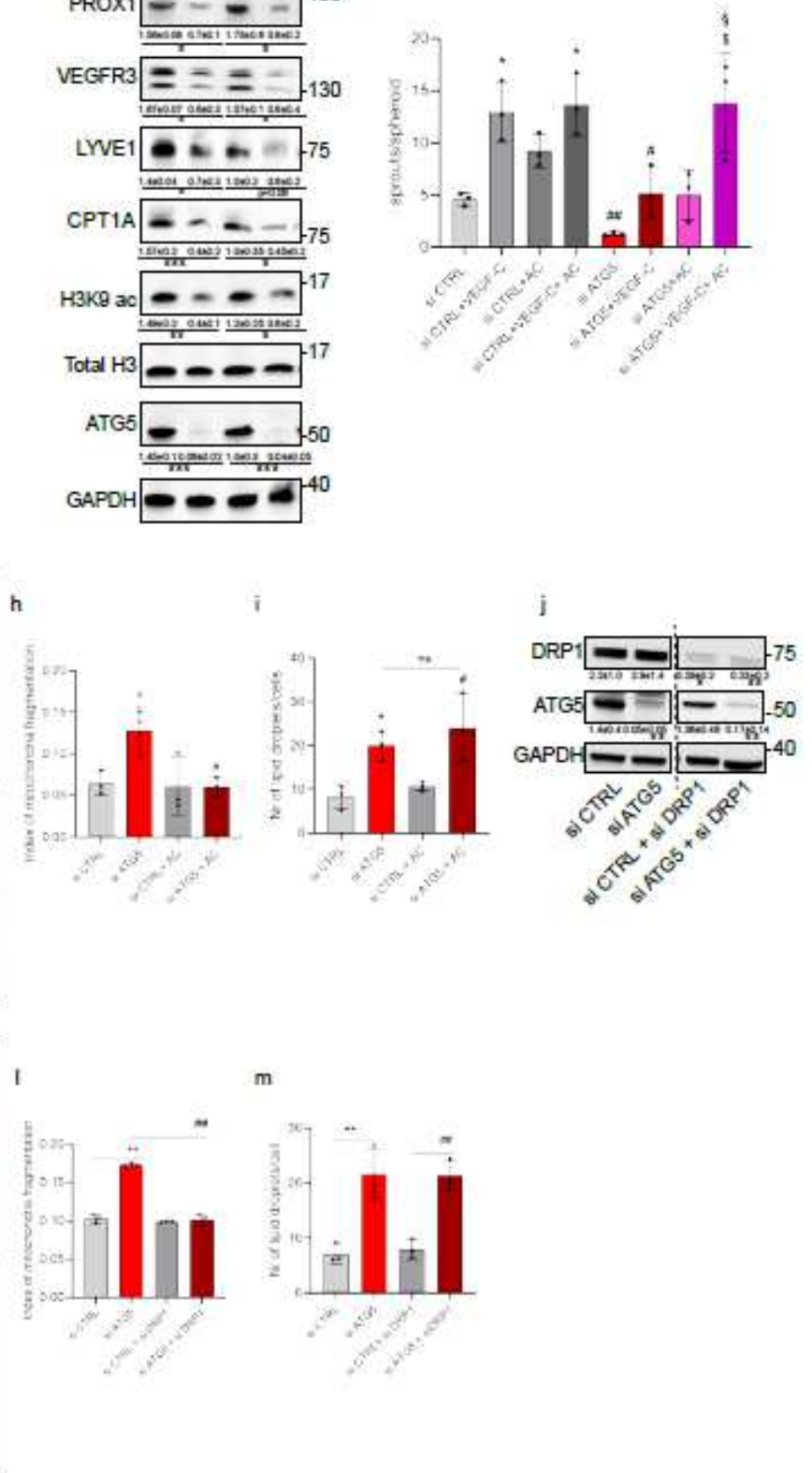

Figure 6

Please manuscript .pdf for full figure caption 

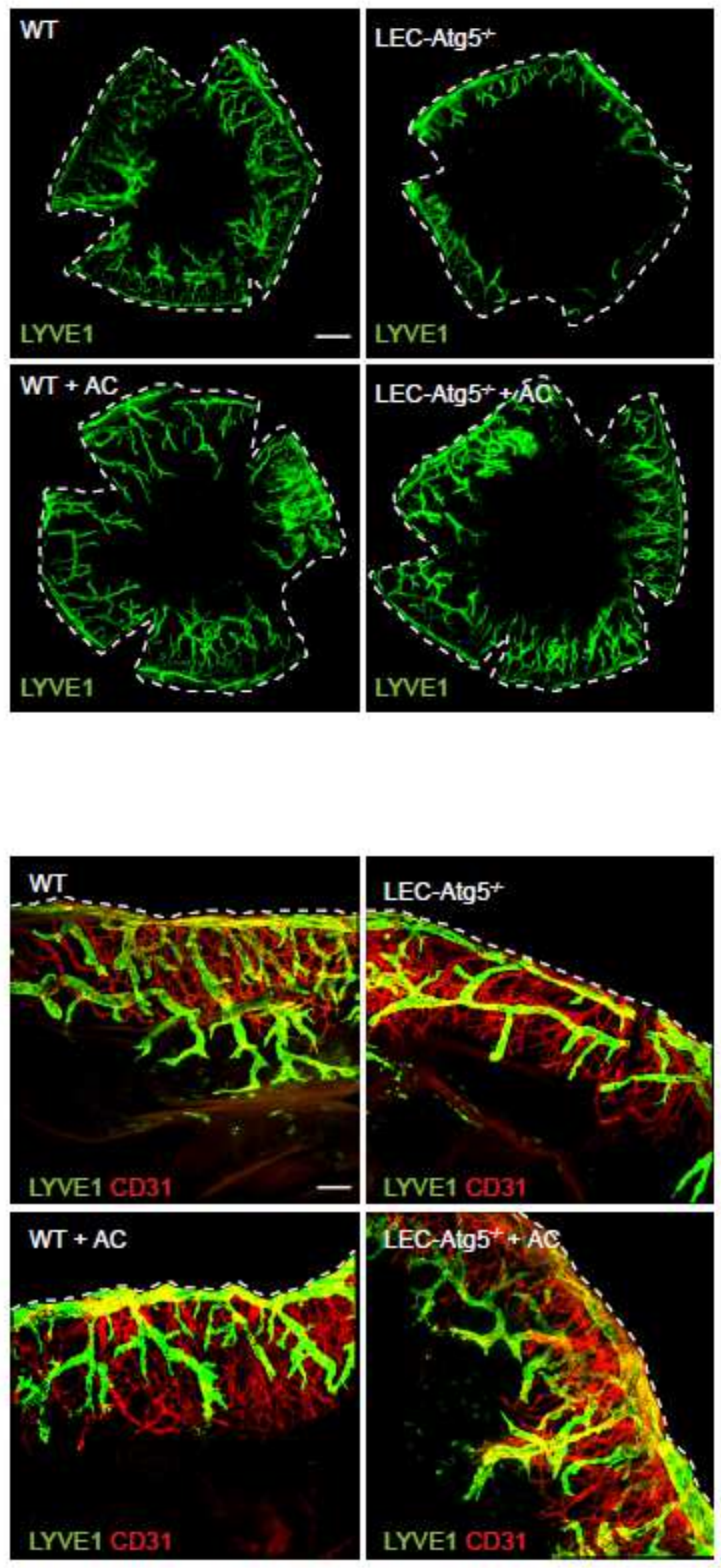

b

c
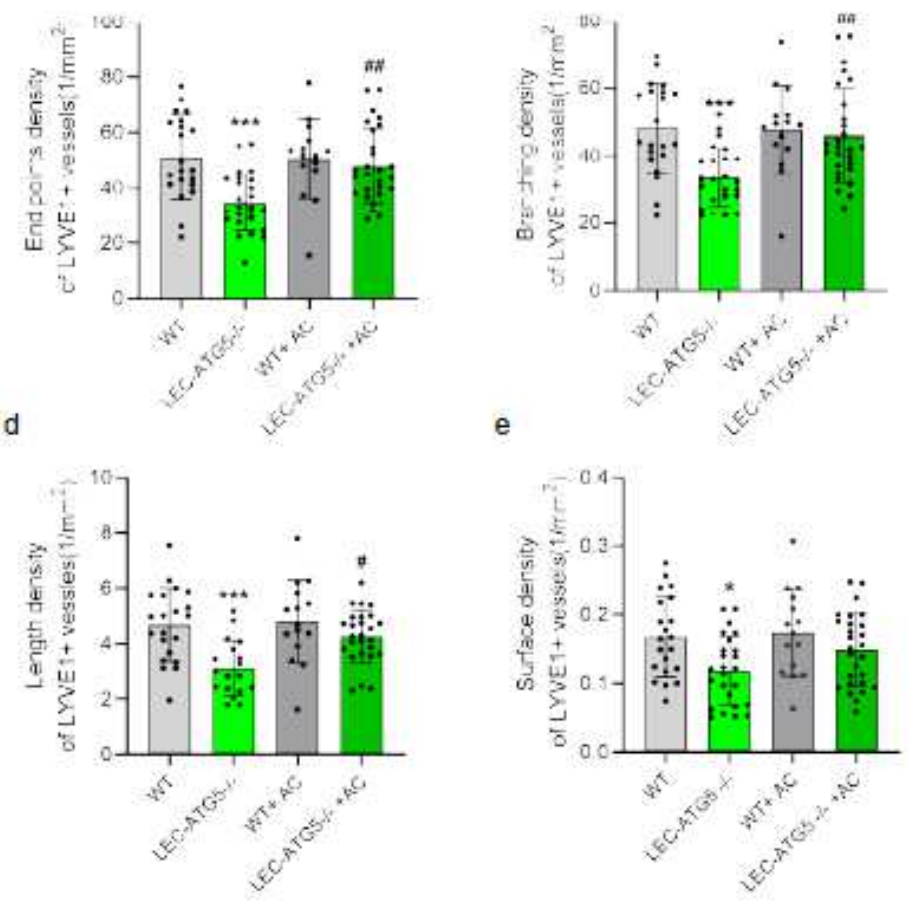

g

h
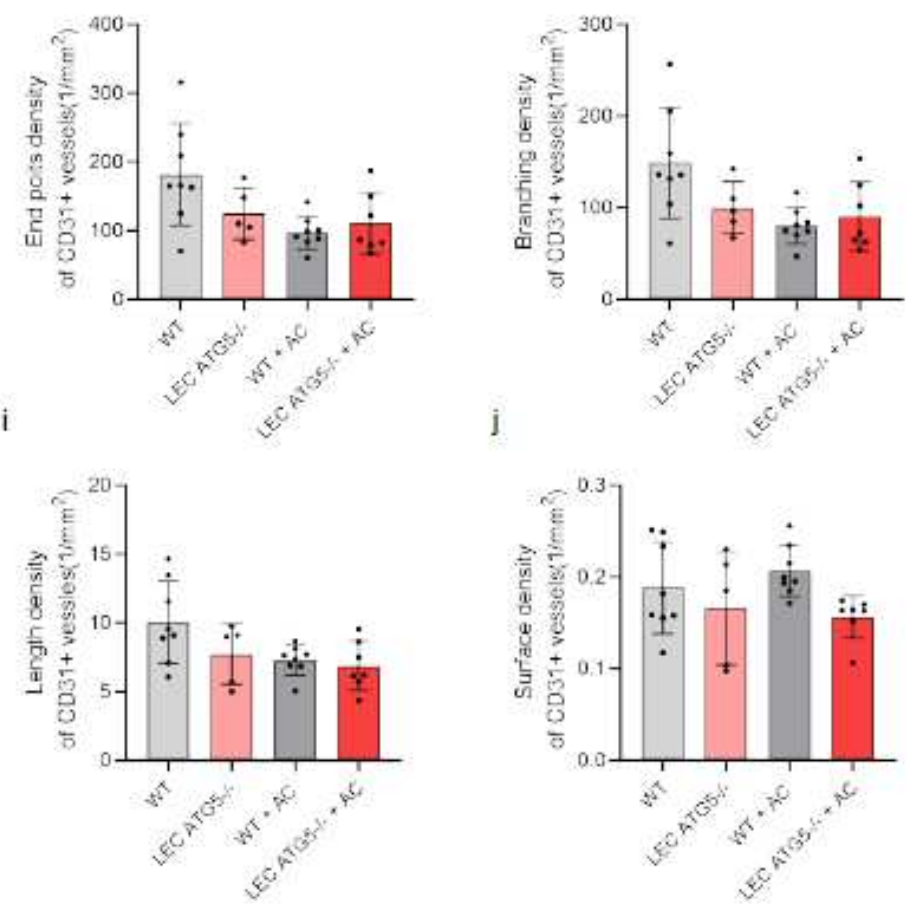

Figure 7

Please manuscript .pdf for full figure caption 


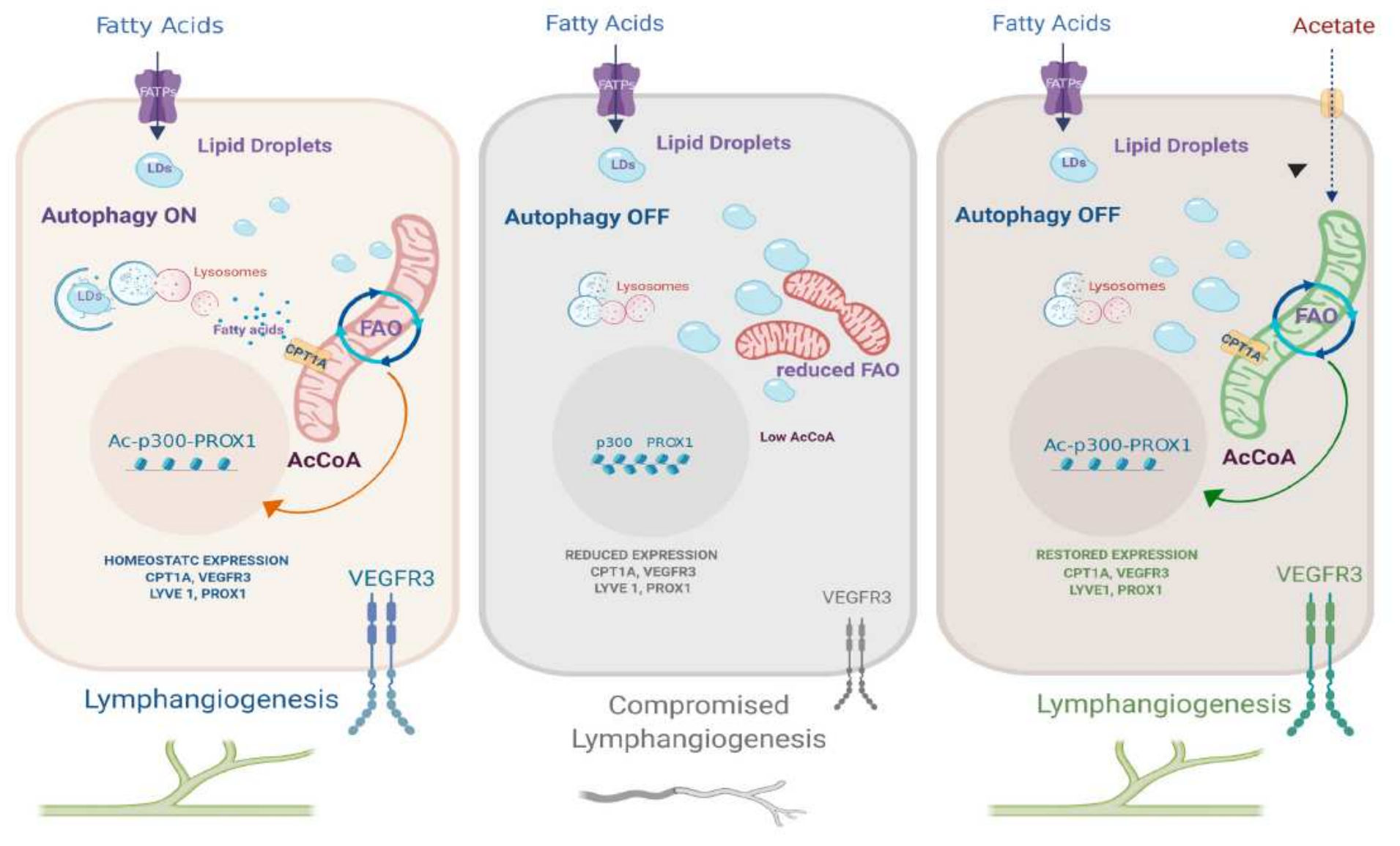

\section{Figure 8}

Please manuscript .pdf for full figure caption 